\title{
CMOS Image Sensors in Surveillance System Applications
}

\author{
Susrutha Babu Sukhavasi ${ }^{1} \mathbb{1}$, Suparshya Babu Sukhavasi ${ }^{1}{ }^{\circledR}$, Khaled Elleithy ${ }^{1, *}$, Shakour Abuzneid ${ }^{1} \mathbb{C}$ \\ and Abdelrahman Elleithy ${ }^{2}$ \\ 1 Department of Computer Science and Engineering, University of Bridgeport, Bridgeport, CT 06604, USA; \\ ssukhava@my.bridgeport.edu (S.B.S.); susukhav@my.bridgeport.edu (S.B.S.); abuzneid@bridgeport.edu (S.A.) \\ 2 Department of Computer Science, William Paterson University, Wayne, NJ 07470, USA; elleithya@wpunj.edu \\ * Correspondence: elleithy@bridgeport.edu; Tel.: +1-203-576-4703
}

check for updates

Citation: Sukhavasi, S.B.; Sukhavasi, S.B.; Elleithy, K.; Abuzneid, S.; Elleithy, A. CMOS Image Sensors in Surveillance System Applications. Sensors 2021, 21, 488. https:// doi.org/10.3390/s21020488

Received: 21 December 2020 Accepted: 8 January 2021 Published: 12 January 2021

Publisher's Note: MDPI stays neutral with regard to jurisdictional clai$\mathrm{ms}$ in published maps and institutional affiliations.

Copyright: $(\odot 2021$ by the authors. Licensee MDPI, Basel, Switzerland. This article is an open access article distributed under the terms and conditions of the Creative Commons Attribution (CC BY) license (https:// creativecommons.org/licenses/by/ $4.0 /)$.

\begin{abstract}
Recent technology advances in CMOS image sensors (CIS) enable their utilization in the most demanding of surveillance fields, especially visual surveillance and intrusion detection in intelligent surveillance systems, aerial surveillance in war zones, Earth environmental surveillance by satellites in space monitoring, agricultural monitoring using wireless sensor networks and internet of things and driver assistance in automotive fields. This paper presents an overview of CMOS image sensor-based surveillance applications over the last decade by tabulating the design characteristics related to image quality such as resolution, frame rate, dynamic range, signal-to-noise ratio, and also processing technology. Different models of CMOS image sensors used in all applications have been surveyed and tabulated for every year and application.
\end{abstract}

Keywords: CMOS image sensor; surveillance systems; resolution; dynamic range; frame rate; signalto-noise ratio

\section{Introduction}

Nowadays, humankind is more dependent on technology, especially in automotive, military, space, wireless sensor networks, and the internet of things for surveillance purposes. To make life easier, these fields have used a lot of convenient methods. We need to think of imaging technology for emerging imaging systems in all the mentioned applications over the past decade. The improvements and advancements are still going on to miniaturize these applications with high speed and high performance for incorporation in a micro area. Due to their amazing performance advantages over CCDs, CMOS image sensors (CIS) have grabbed huge attention in most applications from the past decade. To explain CIS's significance, we herein review the literature since 2009 in which applications were developed using CIS.

CIS is implemented in applications like HODET [1] in intelligent surveillance systems (ISS), Fish Eye [2] in the automotive field, KINECT-KII [3] in the internet of things (IoT), CUBESAT and MENZ SAT [4] in space, IPASS [5] in the military, TIGERCENCE [6] in wireless sensor networks (WSN), etc.

In our survey, we concentrate on literature related to the types and applications of CIS in various demanding areas. Our contributions are listed below:

- We have conducted the first state-of-the-art comprehensive survey on CIS from an applications' perspective in different predominant fields, which was not done before.

- A novel taxonomy has been introduced by us in which work is classified in terms of CIS models, applications, and design characteristics, as shown in Figure 1 and Appendix A Table A1.

- We have noted the limitations and future directions and related works are highlighted.

A novel taxonomy and CMOS image sensor types are discussed in Section 2. In Section 3, CMOS image sensor applications are classified with respect to their different application fields. The design characteristics of CMOS image sensors are explained, and corresponding 
functioning parameters are tabulated in Section 4. Limitations are discussed in Section 5. In Section 6, conclusions are offered.

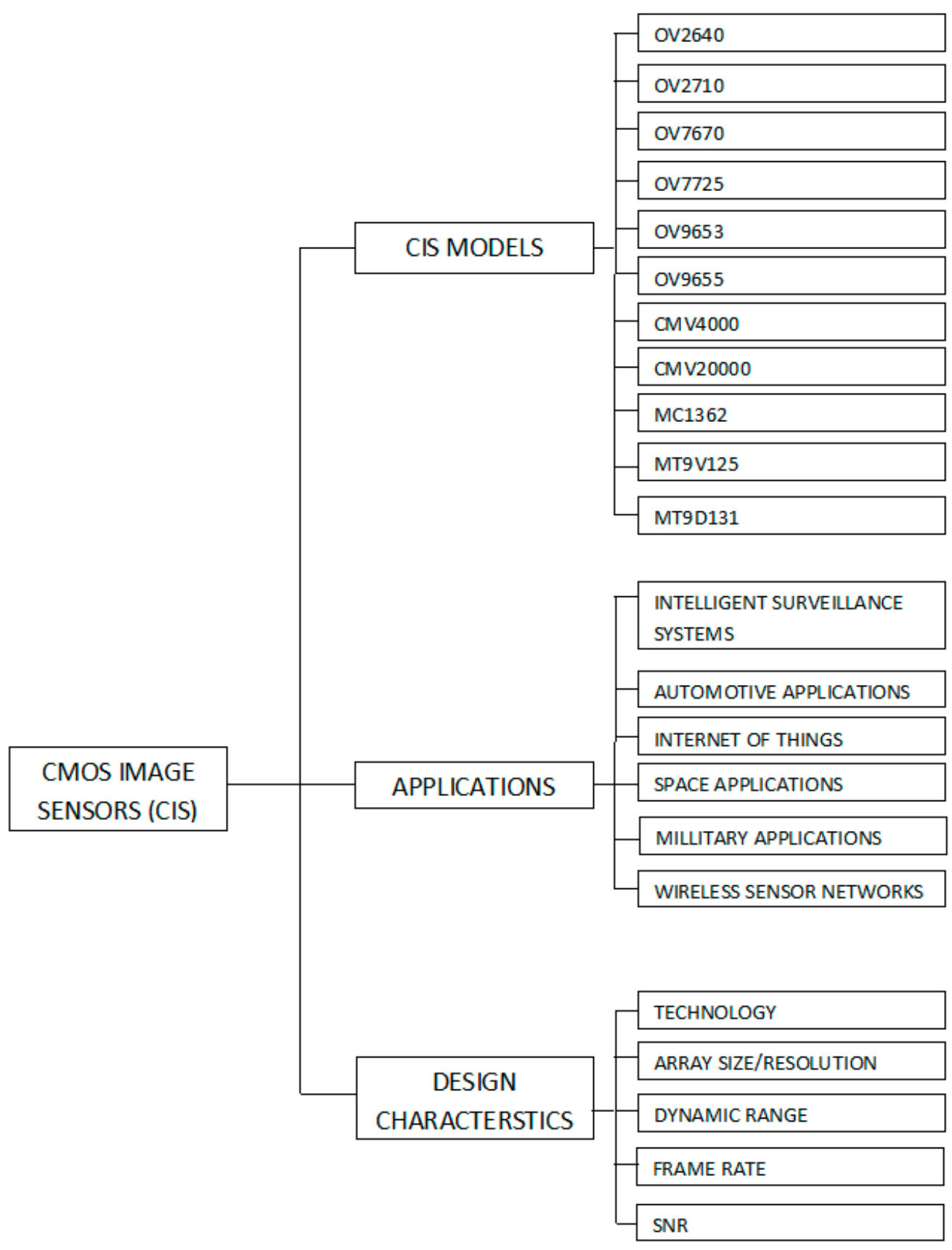

Figure 1. Taxonomy of CMOS image sensor applications.

\section{Taxonomy and Related Work}

We discuss the CMOS image sensor, types of CMOS image sensors, and advantages of CIS over CCD in this section.

\subsection{CMOS Image Sensors and Their Types}

In this section, we discuss the basic CMOS image [7] sensor and its working principle with its applications in surveillance systems is shown in Figure 2. Later the types of the CMOS image sensors are addressed. Finally, the advantages of CIS over CCD are discussed clearly (show in Table A2). 


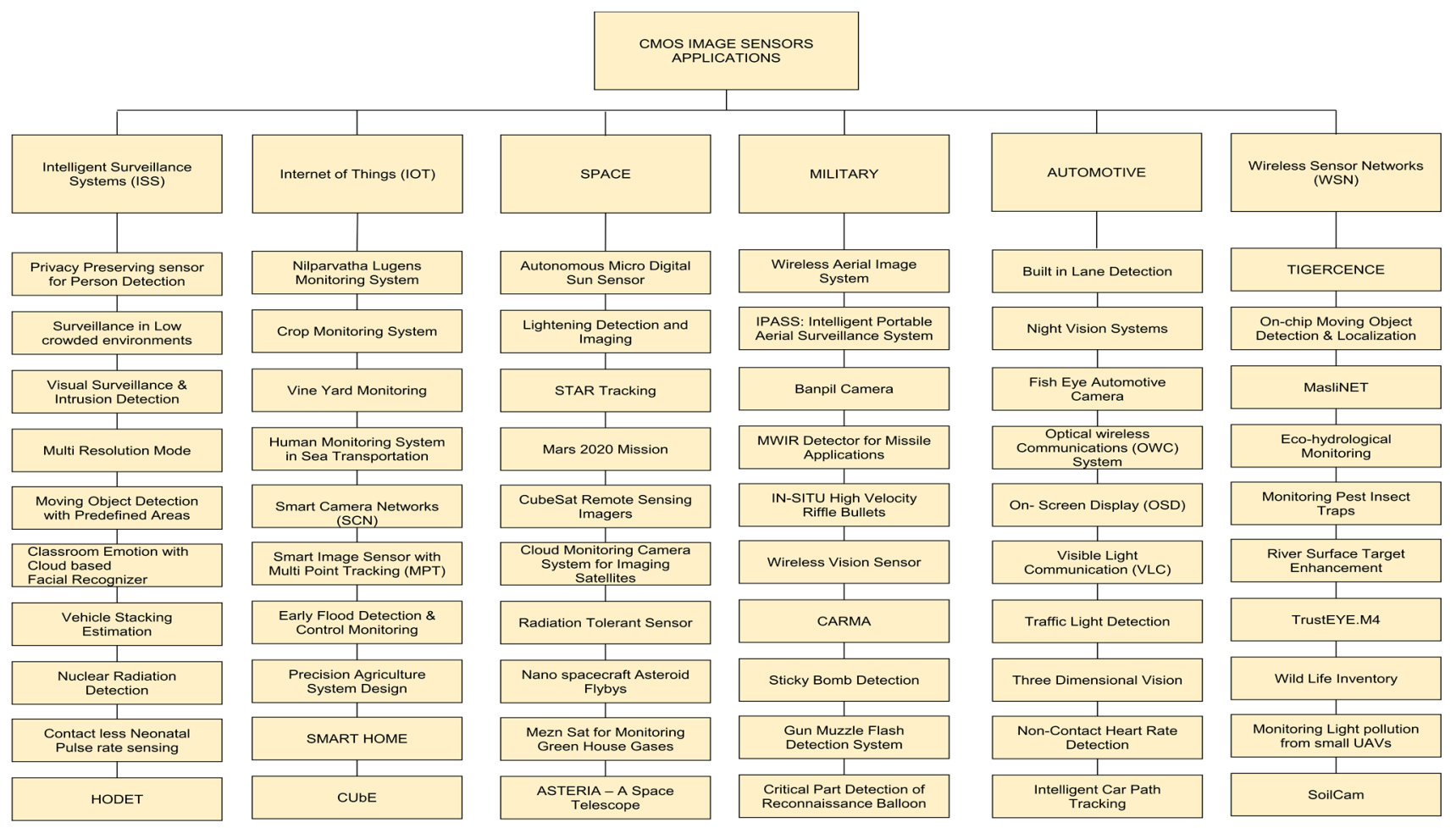

Figure 2. Classification of CMOS image sensor based applications in various fields for surveillance.

\subsubsection{CMOS Image Sensor}

An image sensor is a sensor that converts incident light photons into electrons. CMOS image sensors contain an imaging area, including an array of pixels, readout circuitry and horizontal and vertical access circuitry. The CMOS image sensor architecture is shown in Figure 3 below.

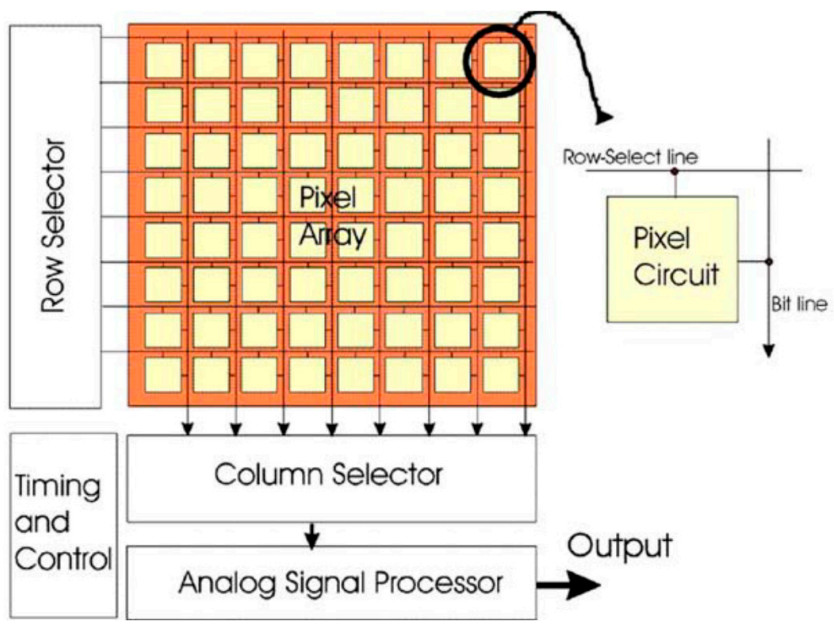

Figure 3. CMOS image sensor architecture. Adapted with from [8] with permission of Elsevier, 2006.

Some traditional imagers are used, namely photodiodes, photogates, and charge coupled devices. Photodiodes convert the incident light into electrons or an electrical current. The current will be produced when photons are extracted into the photodiode. Nowadays, CMOS image sensors use photodiode pixels. Secondly, photogates need to operate at high voltages to collect the electrons generated by incident photons. Thirdly, the charge coupled device, also called CCD, and has architectures involving a combination of parallel and series connection of capacitors. With external circuitry, every capacitor will transfer its collected electric charge to the next capacitor. 


\subsubsection{Pixel Structures}

The pixel structures are two types that were developed earlier in which they created passive pixel sensors (PPS) primarily, and later to increase the quality of an image, they developed an active pixel sensor (APS). PPS and APS's main difference is PPS consists of one transistor in a pixel, whereas APS started with three transistors in a pixel, and later they increased to four transistors in a pixel.

\section{Passive Pixel Sensors}

These are the very first pixel structures used in CMOS image sensors. The circuitry consists of photodiodes, but there is no amplification inside the structure. In these pixel structures, each pixel has a photodiode and a transistor and gets connected to a readout structure. Once the pixel addressing will be done by opening the select row transistor, the pixel will be reset with the bit line and the select row transistor, as shown in Figure 4a.

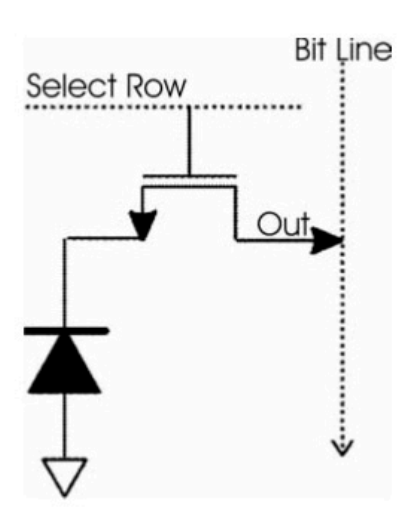

(a)

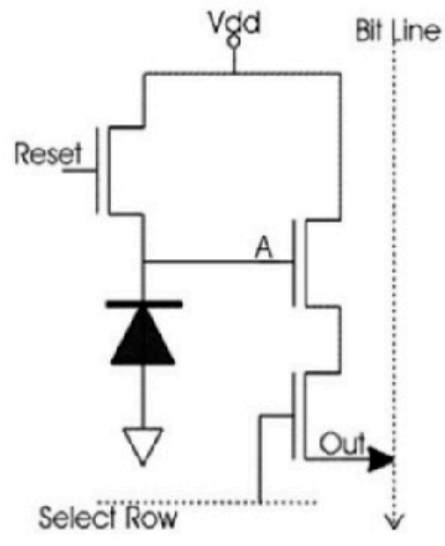

(b)

Figure 4. (a) Passive pixel sensor; (b) Active pixel sensor. Adapted with permission from [8], Elsevier, 2006.

Due to the considerable column's capacitance, high noise and low sensitivity will occur in passive pixel sensors. Because of this phenomenon, the PPS lagged behind the active pixel sensors and made the active pixel sensors to lead the pixel circuits [8].

\section{Active Pixel Sensors}

In this type of sensor, an amplifier is incorporated into the pixel to increase the pixel performance. This amplifier is nothing but a source follower, which is inactive during the state of readout only, as shown in Figure $4 \mathrm{~b}$. The dissipation of power is low compared to the conventional CCD's.

A few drawbacks are also present in the APS, which includes high fixed pattern noise, which occurs because of changes in the wafer process that lead to variations in the transistor's threshold level and gain. The remedy to reduce this fixed pattern noise (FPN) is the introduction of a sampling circuit named correlated double sampling (CDS) circuit. This CDS circuit ultimately reduces the video background offsets caused by the variations in the threshold level [9].

\subsubsection{CIS Types}

Different types of CMOS image sensors have evolved to overcome the drawbacks and meet the application requirements. Some of the types of CMOS image sensors are backside illuminated CIS, logarithmic CIS, high-speed CIS, global shutter CIS, smart CIS, full well capacity CIS, ion image sensor CIS, neural network CIS, pH CIS, low noise CIS are shown in Figure 5. 


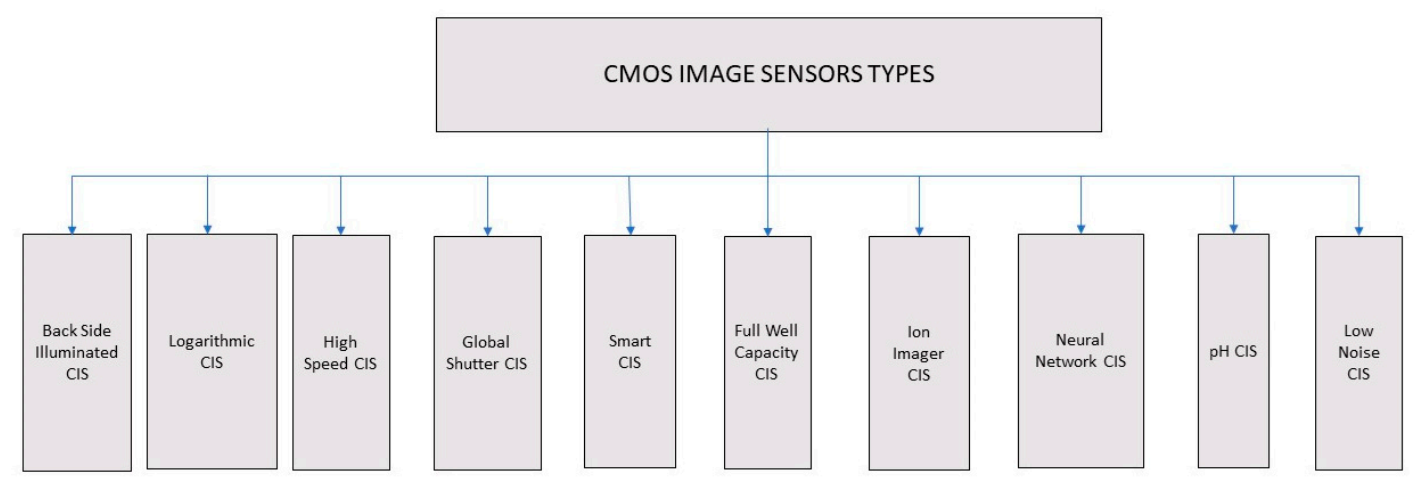

Figure 5. Types of CMOS image sensors.

2.1.4. Advantages of CMOS Image Sensors over Charge Coupled Devices

- Generally, CCD sensors require specific fabrication, a dedicated and expensive manufacturing process. In contrast, CMOS image sensors are made using standard manufacturing facilities and can be made at a very low cost.

- $\quad$ The pixel architectures, i.e., APS, usually consume significantly less power, which is a hundred times less than CCD sensors. This parameter makes CMOS image sensors build compact applications that depend on batteries such as cell phones, laptops, etc. However, CCD applications consume immense power due to capacitive devices requiring more clock swings and control signals externally. And also, to operate, CCD systems require voltage regulators with additional power supplies a lot.

- Due to faster frame rates, CMOS APS architectures have been selective imager component in machine vision and motion estimation applications compared to PPS CMOS and CCDs.

- The other advantage of CMOS over CCD is its high integrability on the chip, which allows digital signal processing functions like image stabilization, image compression, multi-resolution imaging, wireless control, color encoding, etc., to call CIS as smart CIS than CCD.

\section{CMOS Image Sensor Applications}

In this paper, a literature survey was conducted on CMOS image sensors' predominant applications helping humanity in different fields. These fields include automotive, internet of things (IoT), intelligent surveillance systems (ISS), space, the military, and wireless sensor networks (WSNs). The comfortability with and reliance of human beings on these applications are rapidly increasing day by day, as shown in Figures 2 and 6.

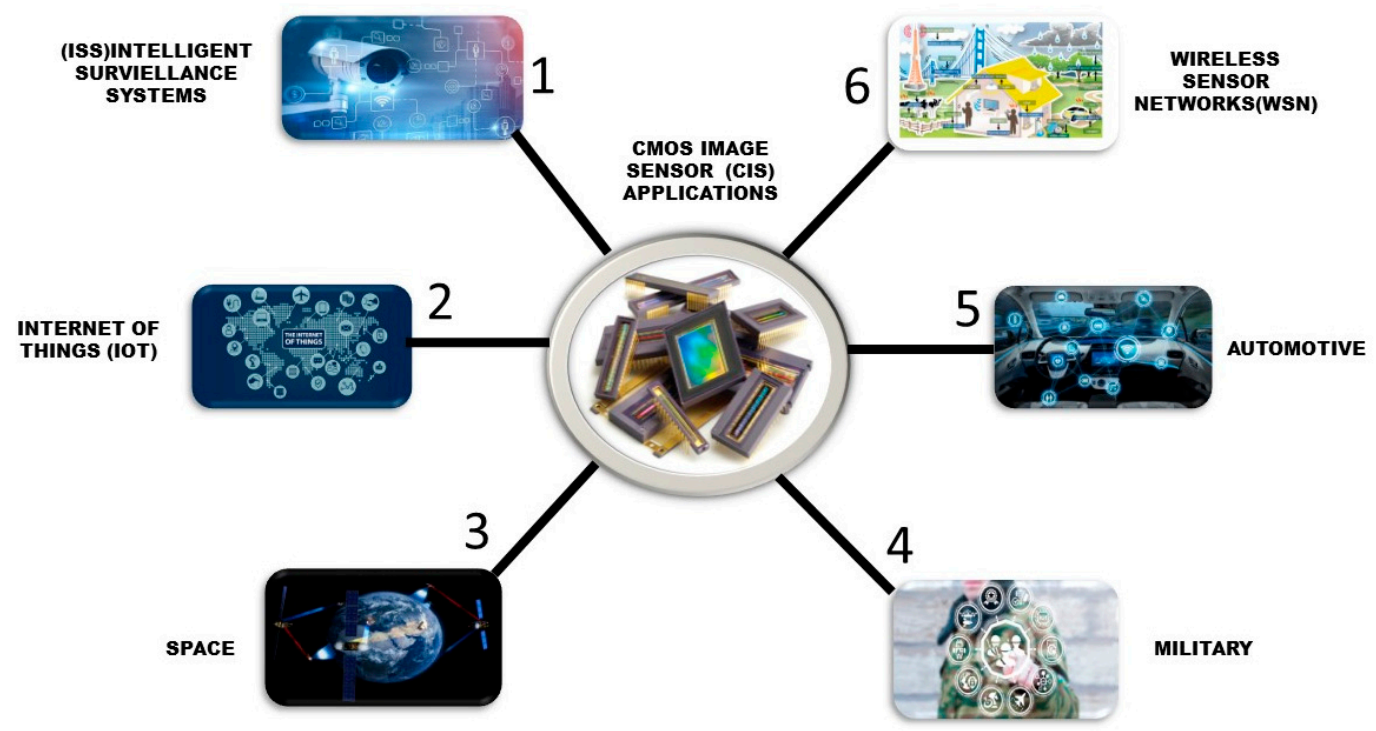

Figure 6. Applications of CMOS IMAGE SENSOR as surveillance system in various fields. 


\subsection{ISS (Intelligent Surveillance Systems) CIS Applications}

Surveillance systems are being part of human lives for safety and security purposes to avoid thefts, attacks and help police departments catch culprits or burglars. However, the cameras cannot be placed in restrooms due to privacy issues, and due to this reason, old age people falling accidents cannot be monitored. Most people feel discomfort about video recording cameras in open public places, so privacy preservation policies are recently requested by people. It is hard to identify the differences between regular cameras and privacy preservation cameras. Nakashima et al. [10] developed a privacy preservation sensor for person detection to identify the person's state and position without capturing and images.

A privacy preservation sensor can detect the person's position by differentiating the background brightness and object brightness in a one-dimensional manner. This sensor can detect the person fallen or standing by keeping it in a vertical position and can identify the person's position by keeping it in a horizontal direction.

Using two sensors in two directions, one can detect both position and the state of the person, as shown in Figure 7.

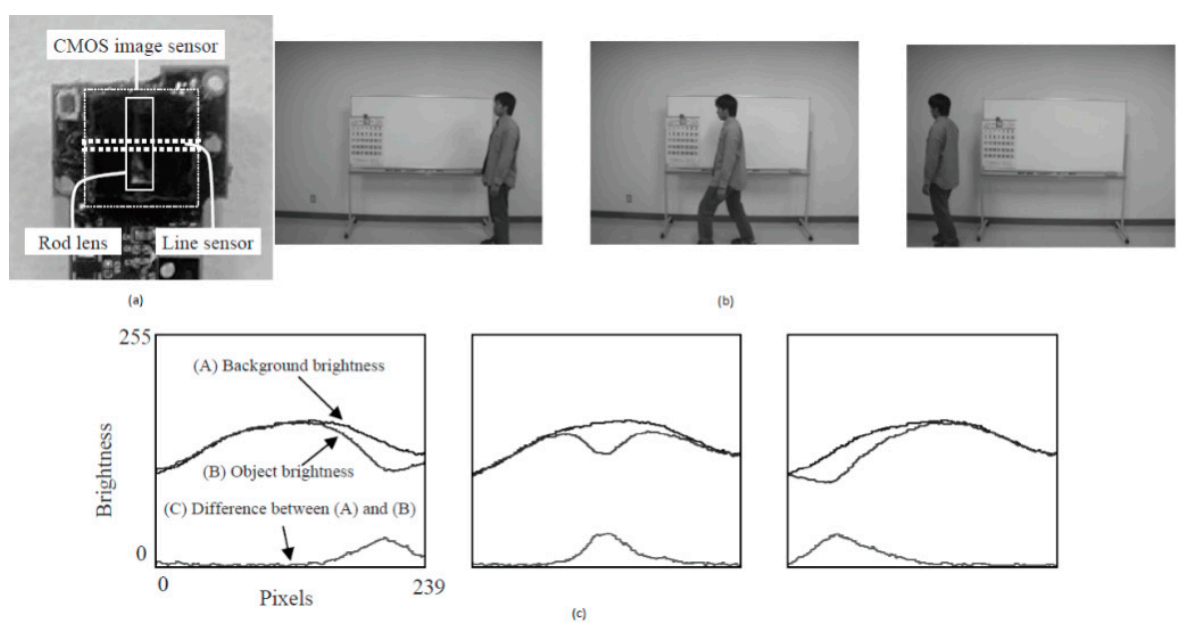

Figure 7. (a) Privacy-preserving sensor for person detection; (b) Field experiment: person detected at the right middle and left side positions; (c) The brightness distributions are made according to the position of the person. Adapted with permission from [10] Elsevier, 2010.

Habibi et al. [11] developed a low-power smart CIS suitable for low crowded environments. The sensor captures the images and detects the image's temporal changes using differential detectors inside the pixel. Person detection in the system can be done by considering pixels with zero difference and will have a high pixel value with the person's edges entered into the frame. The change window inside the circuit will identify the large temporal changes in the pixels and observe its black box approach in Figure 8.

Pham et al. [12] presented a visual surveillance and intrusion detection application network in which small CIS cameras are connected to sensor nodes to obtain visual data for rescue operations, intrusion detection and search operations. Different visual image sensor boards were introduced such as Cyclops [13], Citric [14] SeedEyes [15], Panoptes [16], iMote2 [17], FireFly [18], Eye-RIS [19], WiCa [20] which runs on ad-hoc networks.

This image sensor node uses an UCAM camera for image capture, and a wireless sensor network can indicate an intruder at the image sensor node, using the black box approach shown in Figure 9.

Kim et al. [21] developed the multi-resolution mode CIS for ISS applications shown in Figure 10. This imager can configure its image resolution to a lower level in less monitoring mode and adjust to higher image resolution in high monitoring or secure mode. Thus, adaptability in resolution can save a lot of power for the surveillance system, which is always $\mathrm{ON}$. 


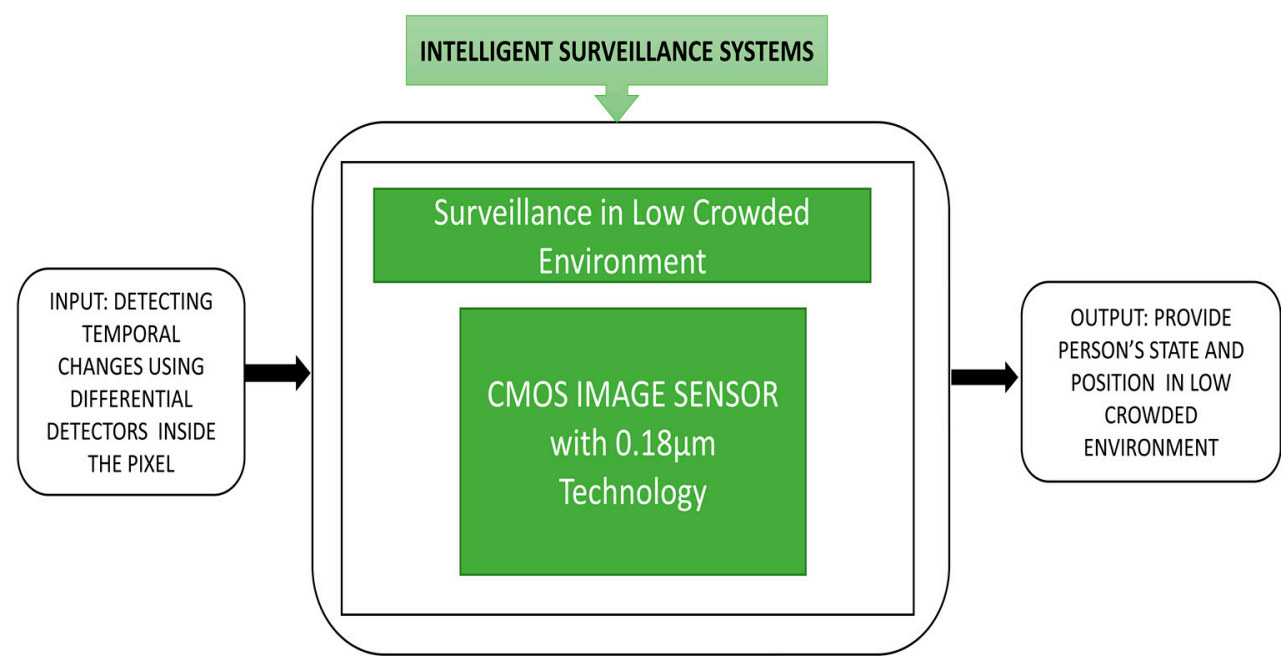

Figure 8. Surveillance in low crowed environment. Empty boxes and illegible text.

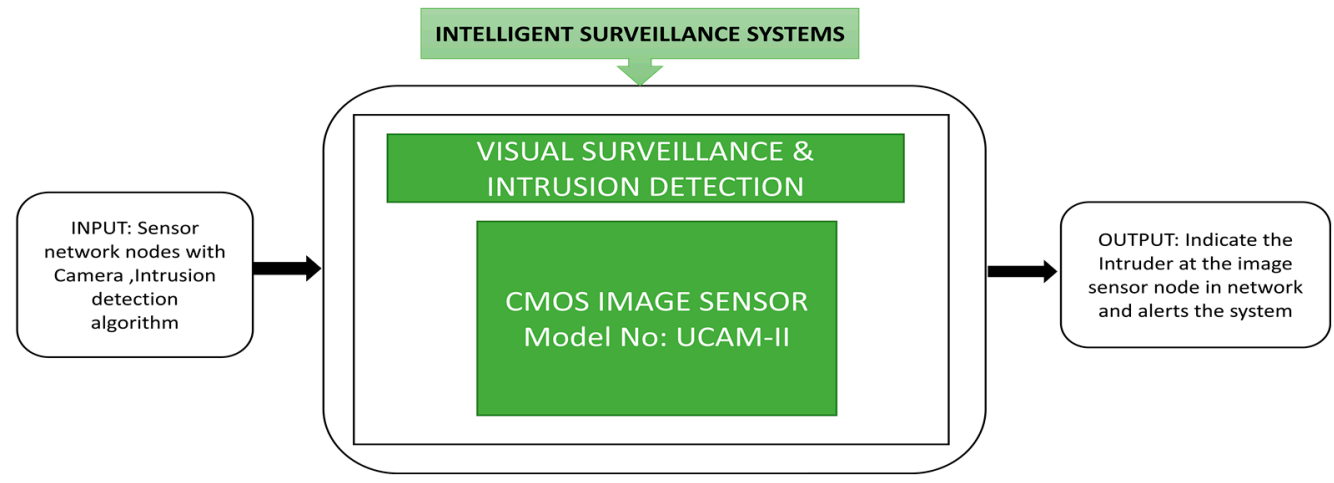

Figure 9. Visual surveillance and intrusion detection application network. Empty boxes and illegible text.

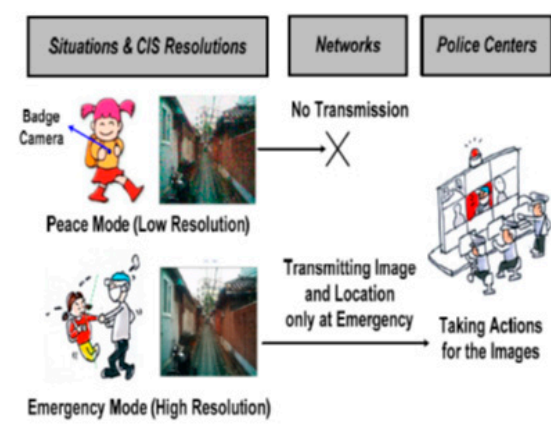

(a)

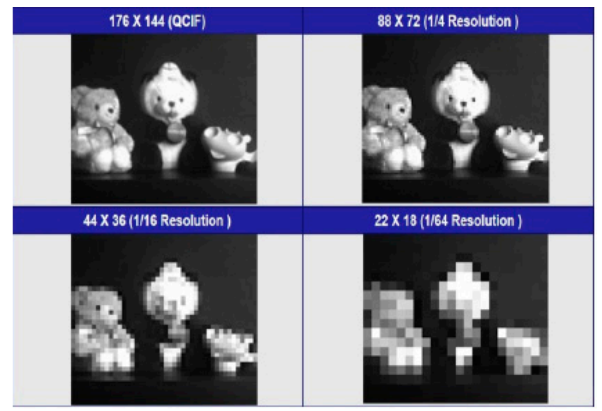

(b)

Figure 10. (a) Surveillance modes-peace mode, emergency mode; (b) Images of different resolution modes [21].

Kumagai et al. [22] proposed a back-illuminated stacked CIS which detects moving objects under different lighting conditions. This imaging sensor has a real-time moving object detection function limited to a predefined range. The event-driven sensor in the imager provides event recording and consumes less power and less bandwidth when operating in low power sensing mode. Emotions and motivations are the two key factors that resemble a positive learning environment. The positive emotion always comes from supportive teaching and good interaction between teacher and students. Learning engagement is a positive classroom emotion, but it is tough for the teachers to monitor the students' emotions. Boonroungrut et al. [23] investigated classroom emotion by using a facial emotion recognition (FER). FER is a technology with cloud-based FER application 
program interfaces for real-time social interactions. Different types of facial expressions like happiness, sadness, surprise, fear, neutral, anger, etc., are detected, and this monitoring was conducted for five weeks, as shown in Figure 11.

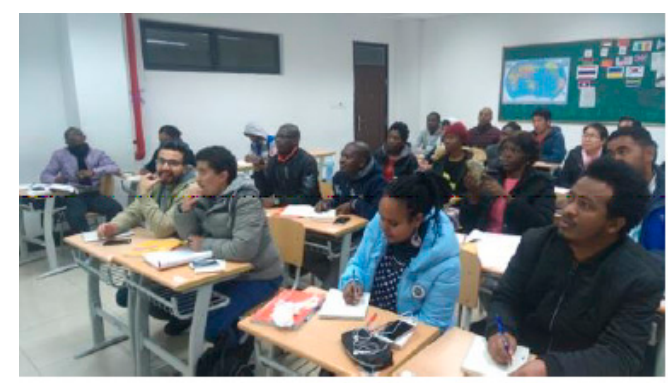

(a)

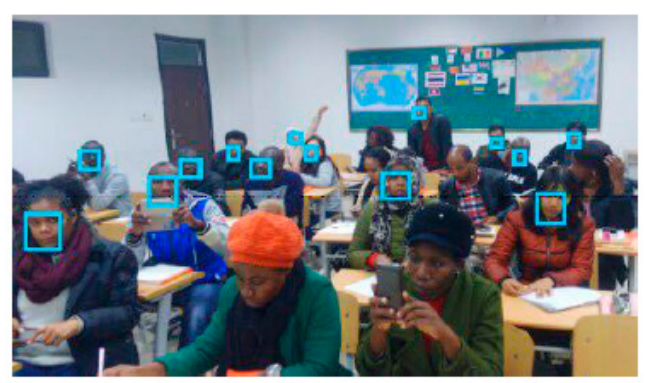

(b)

Figure 11. (a) Image captured in classroom; (b) Captured facial expressions detected using FER technology [23].

Nowadays, traffic jams are becoming a severe problem in metropolitan cities, so various monitoring systems have been introduced to analyze urban development traffic behavior. Freeman et al. [24] developed a novel technique to monitor and capture stable vehicles' images at two different junctions at different times in a day.

Using this system, the traffic will be easily monitored, and vehicle emissions that affect the air quality can be detected without using observers to count the vehicles and classify them in different places. This drone flight is a part of an intelligent traffic system. These flights are sent to the north and south highways of Kuwait and the distance between the vehicles observed and calculated. They are thereby categorized with respect to their year, model, and manufacturer to predict the presence of vehicles for air quality monitoring purposes as shown in Figure 12.
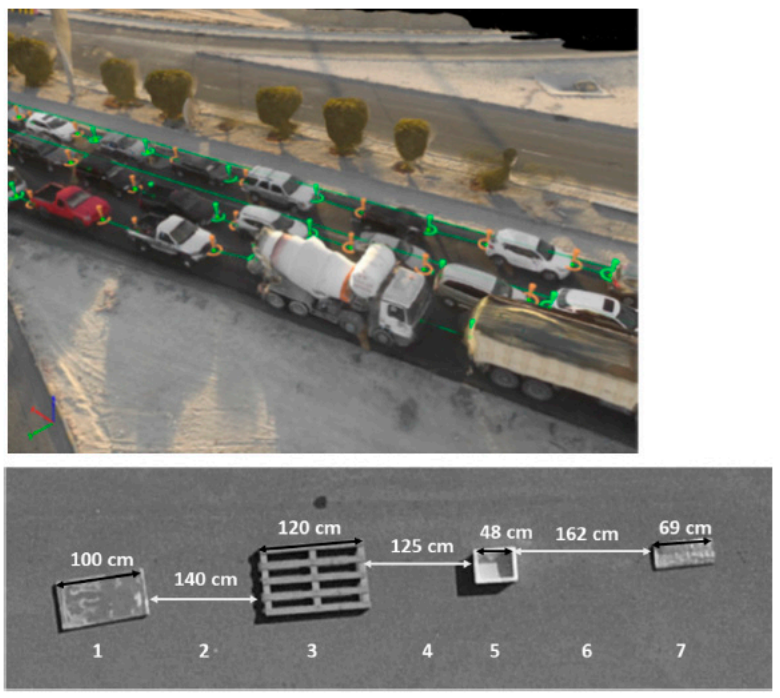

(a)

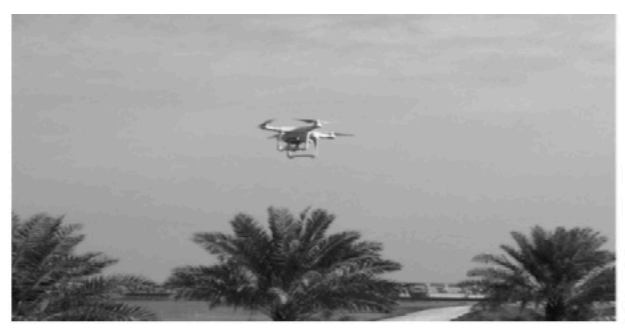

(b)

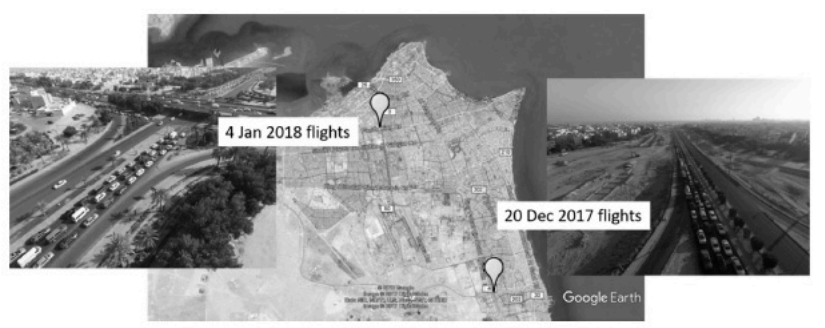

(c)

Figure 12. (a) Captured image of traffic on the Kuwait highway; (b) Drone Flight; (c) Flight locations in north and south Kuwait [24].

Nuclear energy is a rapidly growing technology in the current world. Numerous experiments have been conducted to detect nuclear radiation evolving from nuclear experiments. Nuclear radiation can be detected by using gas detectors, semiconductors, and scintillation detectors. The importance of nuclear radiation detection is obvious, and it has been used in airports, seaports, and land border check posts. Usually, dedicated radiation 
detection systems are expensive and cannot be kept in public places. CIS plays a prominent role in nuclear radiation detection.

Yan et al. [25] developed an uncovered CMOS camera that can detect nuclear radiation while working in surveillance mode, as shown in Figure 13a. Videos are recorded using this camera with a volunteer person wandering around the room under the camera's view. Bright blotches were detected due to the radiation particles exciting the eletrons and can be seen as blotches in Figure 13b,c. Whenever the electrons are excited due to the radiation particles and CMOS sensor visible light, bright blotches are captured by using the CMOS camera.

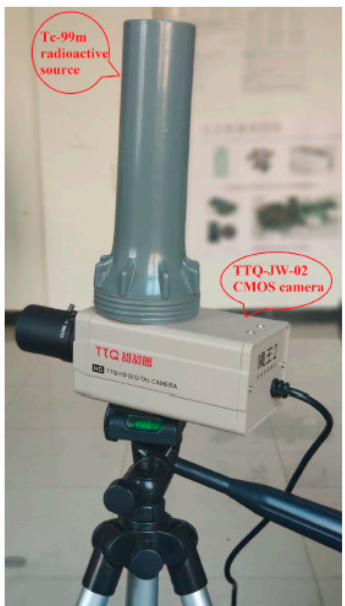

(a)
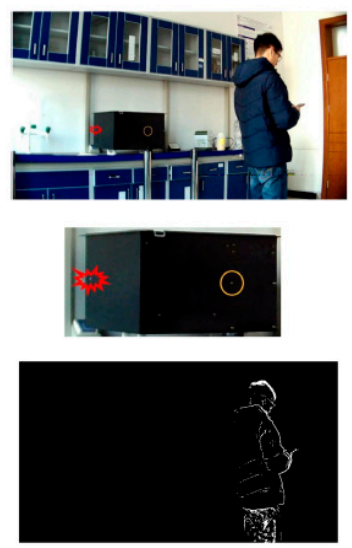

(b)
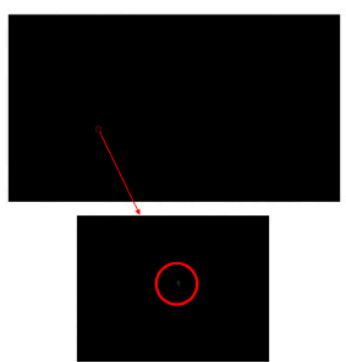

(c)

Figure 13. (a) CMOS camera used for nuclear radioactive signal detection; (b) Field experiment; (c) Radiation bright blotch. Adapted from [25] with permission of Elsevier, 2020.

By introducing CIS into the medical field surveillance, a drastic development change is made in monitoring neonatal, premature babies who require continuous monitoring until their organs in the body achieve normal operation. Many techniques were already implemented to monitor neonates by sticking the invasive electrodes on their body, which can cause skin infections later.

To avoid this, Paul et al. [26] suggested a noncontact sensing method for pulse rate detection, represented in the black box approach shown in Figure 14, in which neonatal monitoring is performed by using CIS-based video cameras and light. This contactless monitoring to analyze the vital signs of neonatal can be done remotely. In this phenomenal monitoring unit, premature babies will have more advantages by avoiding the cables and invasive electrodes on the body.

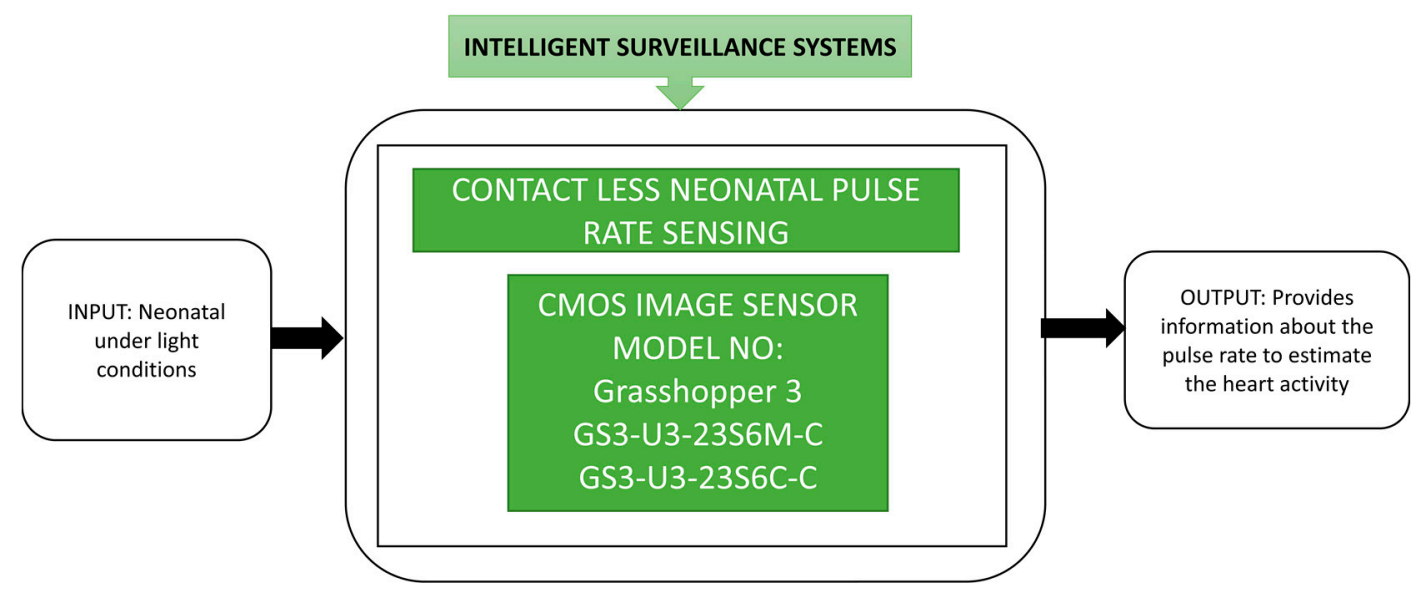

Figure 14. Non-contact neonatal monitoring system. Empty boxes and illegible text. 
Demand has increased for proficient, effective, and dependable monitoring systems for surveillance to sustain the situational awareness in military missions, public safety, battlefield monitoring, and natural disaster early detection monitoring and recovery. Optical video surveillance is not a suitable approach to monitor such applications though it is popular. st. Cyr et al. [1] proposed a new Hybrid Object DEtection and Tracking method named "HODET" using millimeter-wave radar, a visual sensor. It is a computing device to perform object detection, identification, and tracking by using image sensors and radar sensors simultaneously. The radar sensor will give an exact distance, whereas the camera can estimate the object distance illustrated in the black box approach shown in Figure 15.

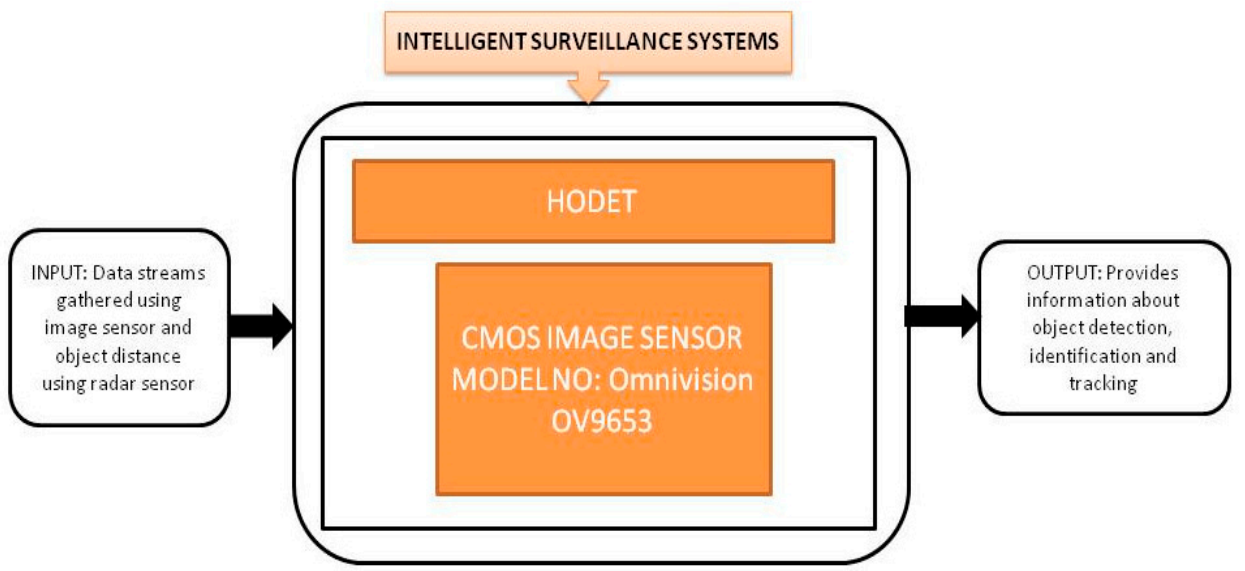

Figure 15. Hybrid Object DEtection and Tracking (HODET).

\subsection{Internet of Things (IoT) CIS Applications}

Over the past decade, a familiar term in all developed countries, and that people rely on it in most essential fields is the "internet of things" (IoT). IoT is a developing informational network that can connect a group of sensors to perform multiple tasks. It is not science fiction or an unnatural thing happening in the industry. It works by using the technical improvements and advancements of various standard devices combined to form a network. For example, shortly, weather jackets will be introduced with an amazing feature that can make the jacket warm in cold weather and cool the hot weather jacket. This is achieved automatically by gathering weather information and sensing the body temperature to maintain the required body temperature by providing hot or cold conditions. A few other existing examples are driverless vehicles, and voice-assisted controlled home appliances.

There is a strong reason to use the IoT in the agricultural field, i.e., lugens is one of the rice pests that damage rice crops on a very large scale in the rice-growing countries of Asia. The rice crop is a crop that is continuously grown in all seasons. Due to lugens every year around one million tons of rice are spoiled. IoT technology is applied to monitor Nilparvatha lugens which transmits viruses like grassy rice stunt and ragged rice stunt. To monitor Nilparvatha lugens, Cai et al. [27] developed a wireless sensor node in the IoT to perform automatic data collection, make real-time decisions, and transmit functions. An OV6620 CIS module is used as a camera module to capture the wireless sensor node design images. As traditional agriculture is changing into modern agriculture today, the IoT plays a crucial role in information gathering and creating wireless sensor networks in the agricultural sector. Zhao et al. [28] proposed a wireless sensor network for agriculture applications. Two types of nodes are implemented and system networking is accomplished in a crop monitoring system. This network was deployed in Beijing and gathered the temperature changes with respect to humidity for $24 \mathrm{~h}$ after switching on the monitoring system as shown in Figure 16. 


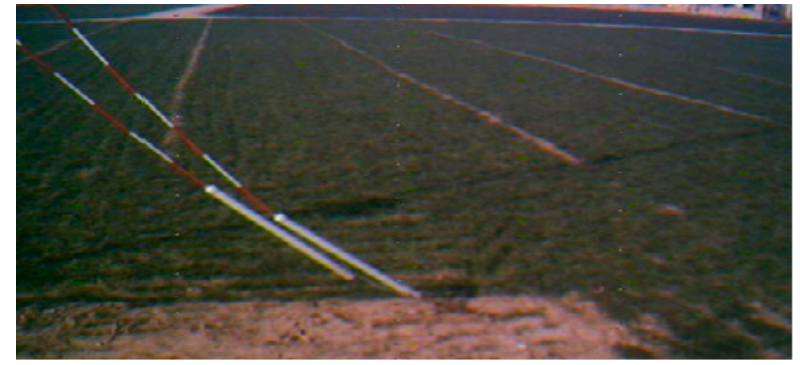

(a)

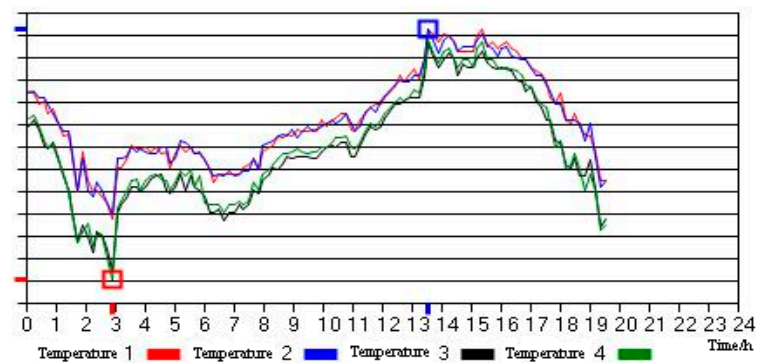

(b)

Figure 16. (a) Captured image of crop monitoring network; (b) Temperature curve changing with humidity. Adapted from [28] with permission of Elsevier, 2011.

Lloret et al. [29] developed a wireless sensor network to monitor vineyard fields. Each sensor node of the wireless sensor network will take images of the field and process the images internally using image processing techniques to identify leaves' uncommon status. Usually, pests, diseases nutrient deficiencies can cause the leaves to appear unusual. If the leaves are identified as defective, then the sensor node will send a message to the sink node via the wireless sensor network to alert the farmer about the plants' problematic status. The diamine putrescine or leaf roll can cause considerable damage to grapes, as shown in Figure 17.

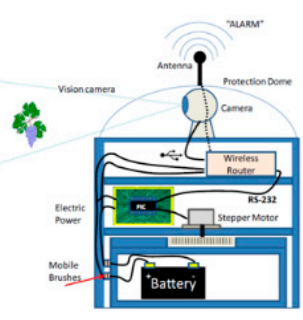

$(\sin$

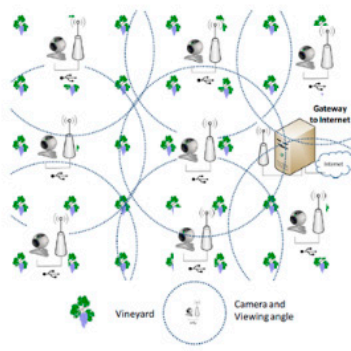

(b)

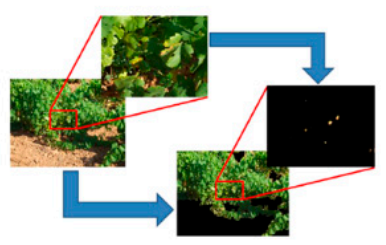

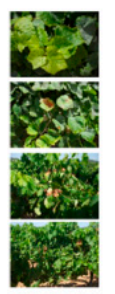

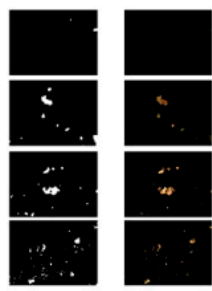

Figure 17. (a) Sensor node for a vineyard monitoring system; (b) Vineyard monitoring by cameras in a wireless sensor network; (c) Detection of brown leaves in vines; (d) Captured images of brown leaves with different sizes taken from different distances [29].

Every year accidents happen in sea transportation due to several reasons like crew drowsiness, mishandling of steering and lack of attention due to crews sleeping at sea traffic. Arima et al. [3] developed a human monitoring system for sea transportation safety. This system aims to identify any small changes in the physical conditions of the people working on the ship during the trip and alert people before the accidents. Facial expressions and electrocardiogram (ECG) data are the key components of this human monitoring system. This system was named the Kinetic Information Integrator (KII) system. This system involves various network cameras and a KINECT sensor, capable of identifying the posture and position of the person's skeleton in three dimensions. Its black box approach is shown in Figure 18.

The face of the navigation officer is monitored during field operation by the KII system. The officer is walking around in the navigation ship to ensure the ship's safety. Network cameras can sense the officer's facial expressions while he is in the sensing range.

Smart camera networks are a newly evolved category in sensor networks that supports high power in network-enabled signal processing. Chen et al. [30] described a low bandwidth wireless camera network platform named CITRIC, suitable for distributed video and image processing and explained its usage in smart camera networks. In the experimental trials, a testbed is used and smart cameras were connected in every contour. The $\mathrm{CN}$ complex concept helps identify the path by communicating with the cameras 
attached at the wireless camera network's contours. A remote control car was sent into the layout and a path obtained by deploying the network to test it. Its black box approach is shown in Figure 19.

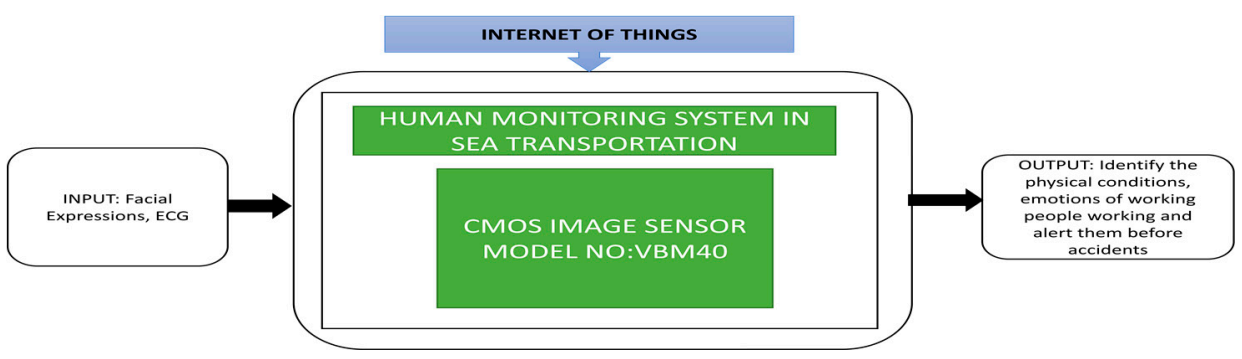

Figure 18. Human monitoring system in the real ship on board monitoring different emotions of the navigational officer.

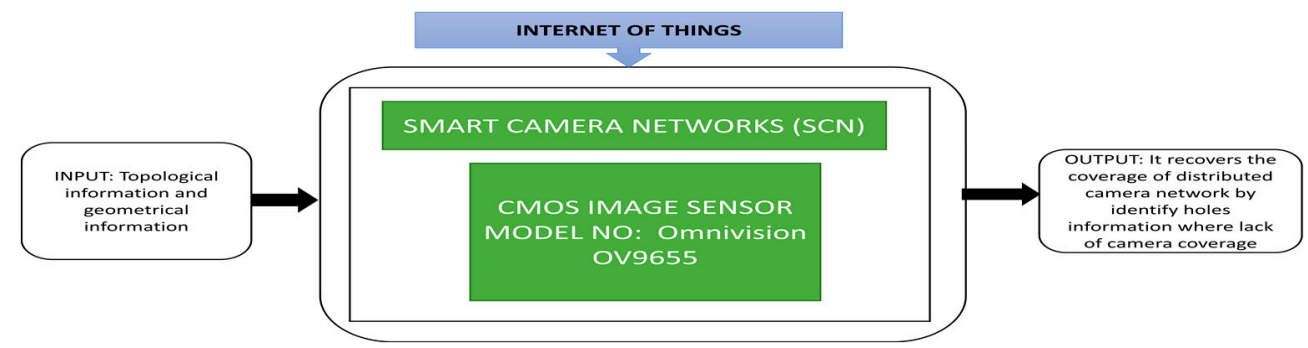

Figure 19. Smart camera networks-based surveillance system with CITRIC mote.

Yin et al. [31] presented a smart image sensor with Array Level Image Signal Processing (ALISP) and Event-Driven Peripherals (EDP) to achieve multi-point tracking (MPT) with edge extraction. The authors presented a prototype setup for an optical handwriting recognition application. The demonstration is done using the letter I in the Chinese language by using the hand fingers, and the image obtained captured by the smart sensor is displayed on a computer by applying the edge extraction technique with the black box approach shown in Figure 20.

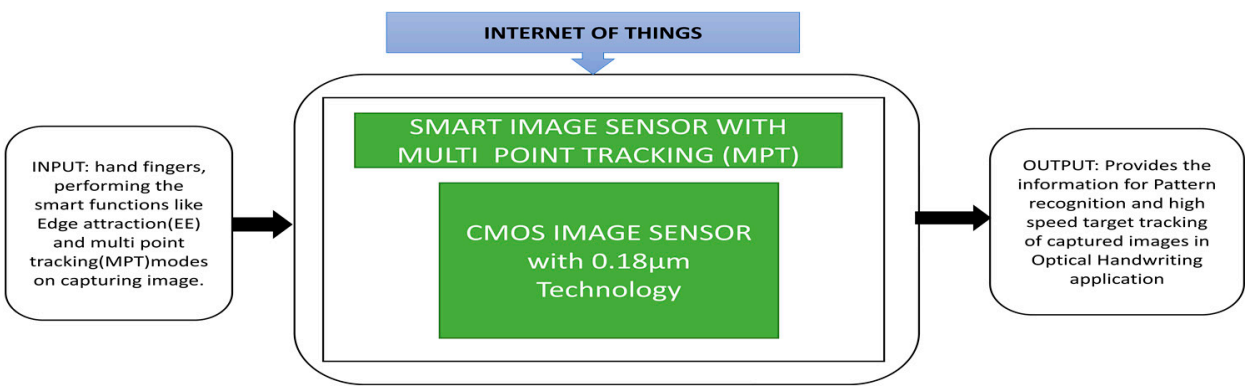

Figure 20. Smart image sensor with Multi Point Tracking (MPT).

Natural disasters like floods occur every year, and due to lack of immediate rescue actions, the rate of peoples' deaths is increasing year by year. During floods, water dams can overflow suddenly into nearby living areas. People often cannot get evacuated immediately, which leads to the sudden death of hundreds of people. Thekkil et al. [32] presented a wireless sensor network-based early flood detection control monitoring system. This system uses CMOS image sensors to gather data in the form of images and transmits these images via wireless sensor nodes to remote monitoring stations using Zigbee and Global System for Mobile (GSM) communication networks. Clients will get an alert from the remote station to take the necessary action to save people in the flooded area. The black box approach is shown in Figure 21. 


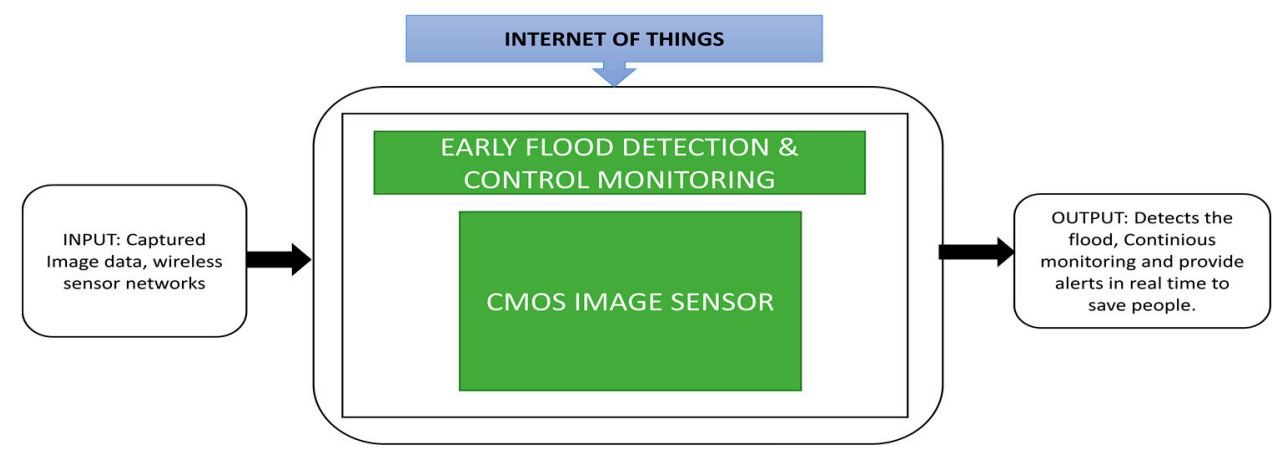

Figure 21. Flood detection and control monitoring system.

The proposed system architecture explains how the interaction happens between remote host and client via wireless sensor nodes, Zigbee and GSM networks. A flood detection and control monitoring system is implemented in the Java software Net Beans, which uses a SIFT algorithm to find the dam's current water level.

Patokar et al. [33] proposed a precision agriculture system design focusing on a monitoring systems that can collect important data, i.e., temperature, soil moisture, humidity, and sprinkler water flow. This data will be sent to a personal computer and necessary decision-making and actions will be done with the help of the internet of things involved. Using information technology in agriculture can help farmers achieve good productivity and soil fertility. Raj et al. [34] developed an automation system using the internet of things that can operate by using voice commands in various languages to control different home appliances. It is a network-based wireless home automation system that uses Google Assistant to communicate users with the devices.

This system is beneficial for old age people and physically disabled people who cannot easily move. This system can be controlled in two languages, and CIS OV2640 is used as a mini camera on an Arduino board, and its black box approach is shown in Figure 22.

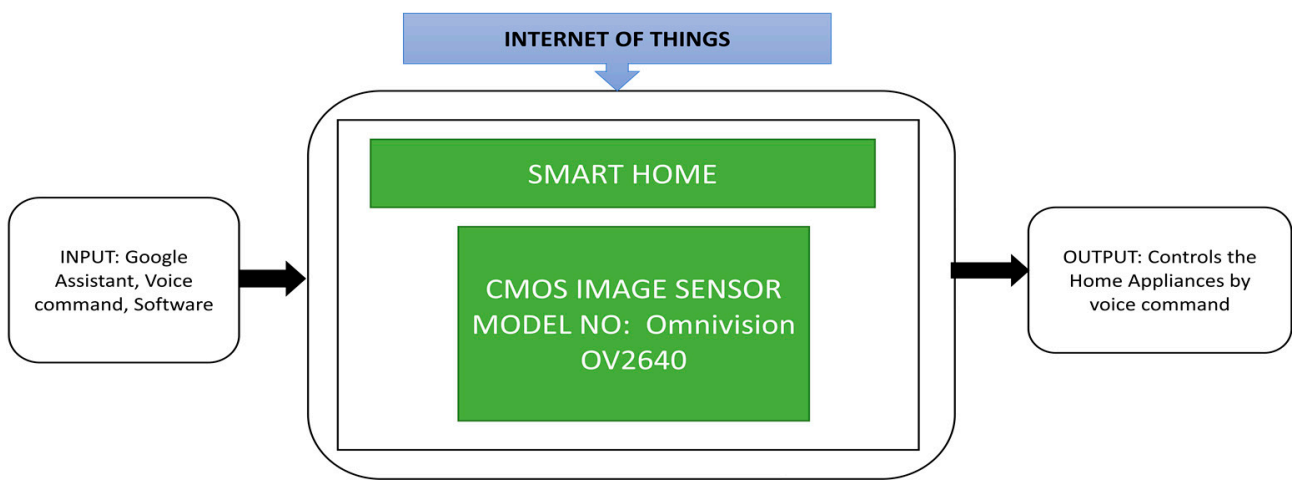

Figure 22. Home automation system.

As the world population is increasing rapidly, this brings challenges in transport for working offices, inside the large organization's route map. Hartmannsgruber et al. [35] introduced Continental Urban Mobility Experience (CUbE), a driverless shuttle for cities. It works on the concept of fleet management to communicate the $\mathrm{CUbE}$ with the users. This CUbE can transport the people from source A to destination B in the allocated area in their work facility. This fleet concept can help users choose the appropriate CUbE in their pick-up location and drop them off at a target destination by sharing the ride with co-passengers traveling along the same routes. To use this facility, they introduced an android application called "Call A CUbE." This application can be downloaded from the Android Play store and allows the users to book rides inside the transit area. This application will also indicate the status of available CUbEs in your area concerning trips 
and timings. Figure 23 presents the black box approach of the CUbE platform, its backend software using fleet management, and an Android application named "Call A CUbE."

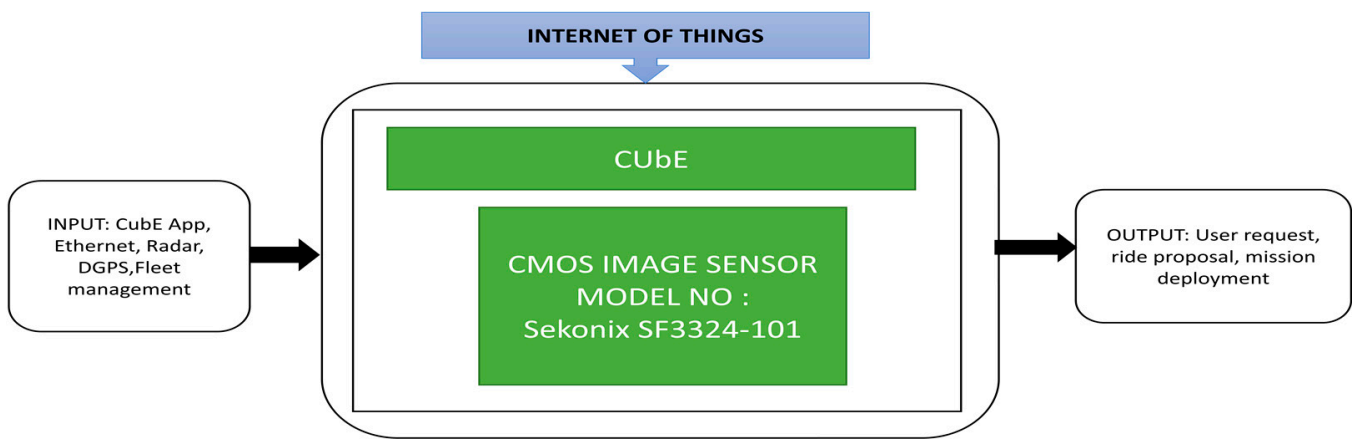

Figure 23. Continental Urban Mobility Experience (CUbE).

\subsection{Space CIS Applications}

CIS are becoming the crucial component in space applications to measure the distance, altitude, and observing the Earth for various parameters like water, ozone levels, etc. Apart from this, CIS are involved in star trackers, rover cameras, satellite monitoring, space station monitoring, etc. CCDs were widely used in space applications earlier. Due to the high power consumption of CCDs, CIS is however, preferred. CIS has a high dynamic range and modulation transfer function, which are the critical components for space applications.

The attitude information is an important parameter to be monitored for a spacecraft. Sun sensors are a kind of attitude sensor that can detect the spacecraft's orientation by observing the Sun's angular position. Xie et al. [36] proposed a Micro Digital Sun Sensor ( $\mu$ DSS) that can be used in microsatellites due to its special functions like low power consumption, radiation hardness, miniature size and high accuracy. The black box approach of a micro digital Sun sensor is shown in Figure 24. This sensor works with two modes of operation, namely acquisition mode and Sun-tracking mode. During the acquisition mode, the Sun's coordinates can be estimated within the determined region of interest. During Sun-tracking mode, the sunspot final centroid coordinates will be determined. This application is applicable to satellites in low orbit that circle around the Earth in ninety minutes. A cross-view of APS+, which is used as an imager in the micro digital Sun sensor is produced and for radiation tolerance, this sensor is covered with an aluminum shield. Another critical parameter in space applications is lightning detection and imaging in the Earth's orbit during thunderstorm observation. This observation gives essential data to estimate the changes in the climate. To analyze the origin of nitrogen oxides origin, lightning should be monitored. The detectors on the ground have an insufficient range which causes less coverage of low-density population regions and oceans.

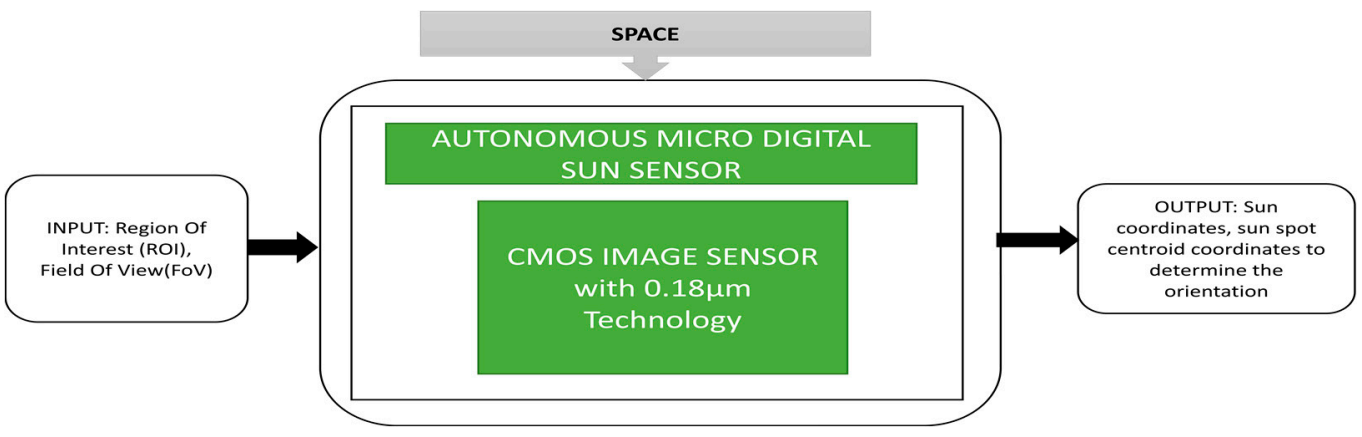

Figure 24. Autonomous Micro Digital Sun Sensor ( $\mu \mathrm{DSS}$ ).

Rolando et al. [37] presented a dedicated CIS for lightning detection and imaging, and evaluated the image sensor chip potential used for lightning detection. The lightning 
imaging sensor instrument and the imager satellite are dedicated to monitoring lightning in the Earth's atmosphere. The lightning imager continuously observes and achieves above $80 \%$ coverage of the Earth's globe and provides real-time lightning detection and geolocalization, and its captured images. The corresponding black box approach is clearly illustrated in Figure 25.

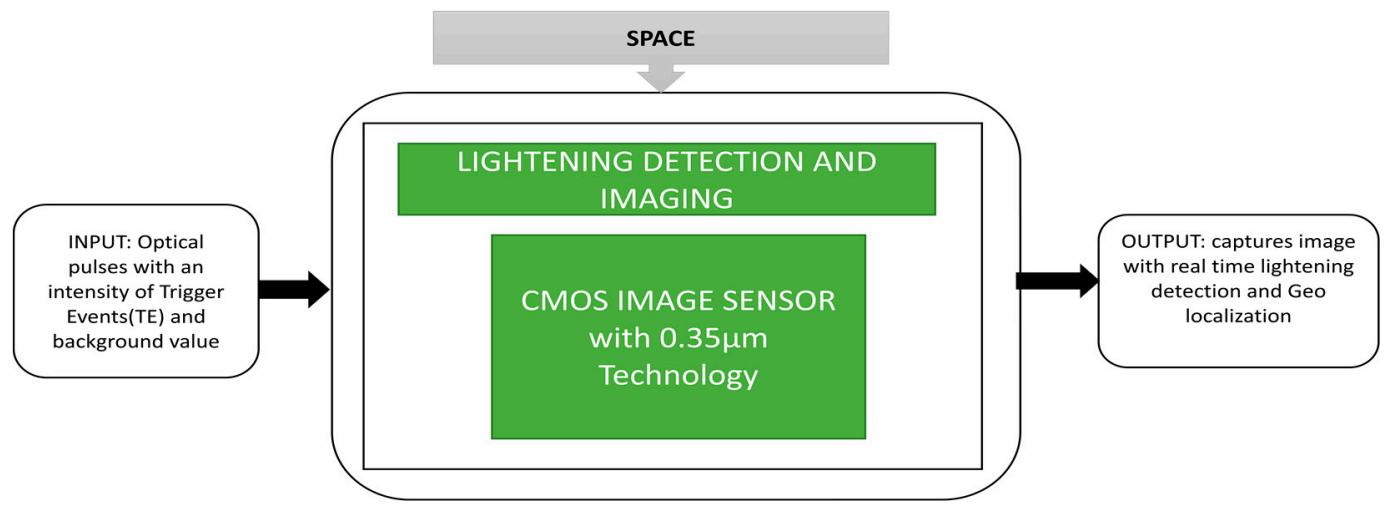

Figure 25. Lightning detection and imaging observation over earth.

Star tracking is one of the space applications to track the stars and their radiation measured by their visible light magnitude. Many changes are being made in space technology in terms of micro- and macrosatellite operations. The satellites dedicated to Earth observation require an automatic attitude control system to drive the satellite to the required destination. Many navigation sensors like gyrosensors, Sun sensors, observation sensors having imagers, star trackers, and Earth magnetic sensors are involved among which star trackers are the most suitable ones. Qian et al. [38] developed an architecture for CIS to apply an adaptive integration time function where brighter pixels are detected and read quickly. Low-intensity pixels will take a long integration time to be detected.

The CIS used here is to capture the starfield scenes where stars are considered the brighter pixels and detected instantly. Its black box approach is described in Figure 26. This imager can prevent saturation and capture images with low power consumption due to its adaptive integration time feature.

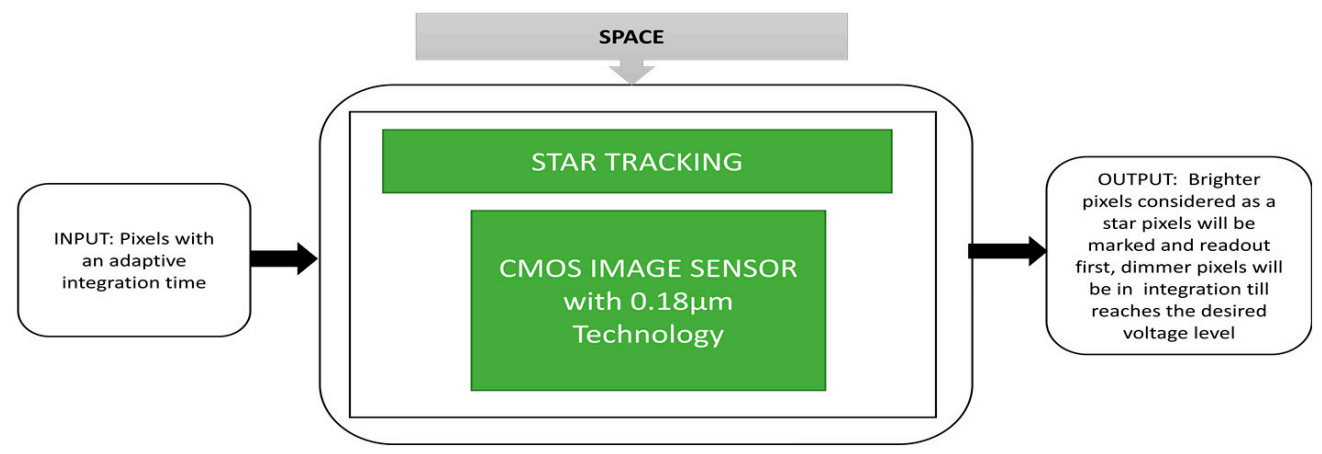

Figure 26. Imaging camera setup for star tracking measurement.

Mckinney et al. [39] proposed an enhanced engineering camera (EECAM) to be used in the 2020 Mars Rover mission. This rover is incorporated with the next-generation system, an upgrade to the earlier engineering cameras used in the Mars Science Laboratory Rover mission and Mars Exploration Rover mission. Earlier generation Hazcams and Navcams were used as engineering cameras in the Mars Exploration Rover mission during 2000. The Mars 2020 enhanced engineering cameras use a $20 \mathrm{MP}$ color CIS instead of the CCDs used in earlier Mars missions, and its black box approach is illustrated in Figure 27. 


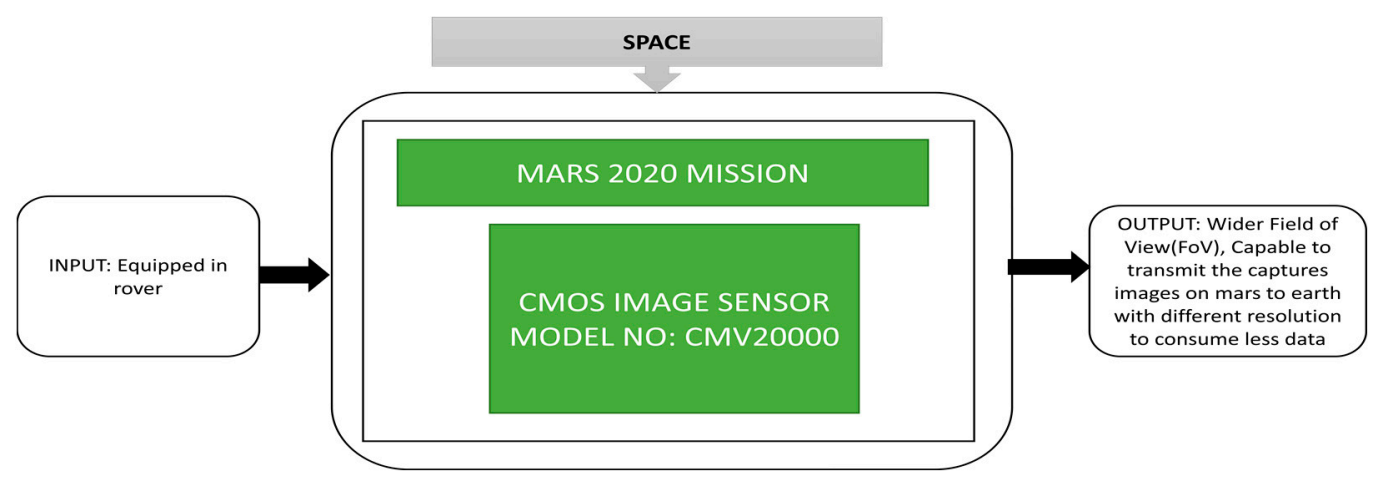

Figure 27. Enhanced Engineering camera (EECAM) using CMOS image sensor CMV-20000, i.e., Navcam.

There are two cameras incorporated into Mars 2020 rover, namely a Navcam and a Hazcam. The Navcam is mounted on a pan/tilt mast and used to capture color stereo panoramic images from two meters height from the Mars surface, whereas the Hazcam is mounted on the rover body and can capture regular color stereo images from a height of $0.7 \mathrm{~m}$ above the Martian surface. A new camera called Cachecam is introduced and can capture sample material images. The greater number of EECAMs being incorporated into the Mars missions will increase the total operating efficiency of the 2020 Mars rover on the Martian surface. This launching of the Mars mission was scheduled for the summer of 2020.

The building of satellites is becoming a critical task for scientists to monitor fundamental components in a rocket and examine the functionalities of each block of the satellite. It increases the manufacturing cost and operating risks during maintenance, but the functionalities are split to reduce costs and balance the risk in operation for CubeSats. Pack et al. [40] described two CubeSat remote sensing missions, which were proposed by the Aerospace Corporation. The Aerospace Corporation developed the CubeSat Multispectral Observation System ("CUMULOS") and R3 sensors, which can be easily incorporated on $3 U$ Cubesats. CUMULUS is used to do environmental and weather missions. Over 19 years, the Aerospace Corporation has launched 20 picosatellites and nanosatellites and has yet to launch a few more in completion stages. CUMULOS is a three camera payload incorporated into the NASA Integrated Solar Array and Reflectarray Antenna (ISARA) mission. It is a mini weather satellite that focuses on low light conditions and the R3 sensor is a CubeSat which focuses on operational land imager instruments. The complete structure of the ISARA spacecraft with antennas and payloads containing three cameras, and its black box approach are clearly explained in Figure 28. There is no additional space required to insert the CUMULOS payloads in the spacecraft, the R3 satellites line of assembly and regions are covered by three CUMULOS cameras simultaneously.

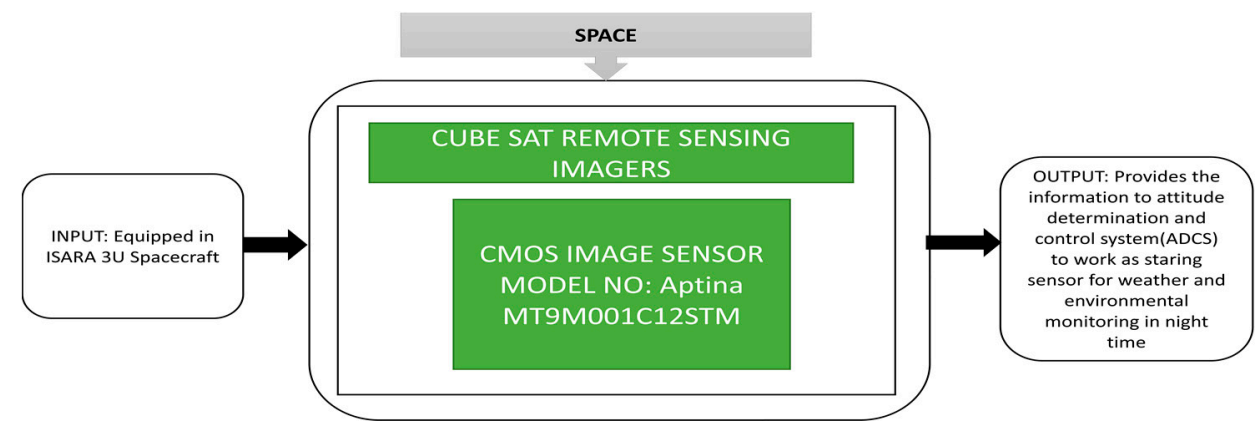

Figure 28. NASA Integrated Solar Array and Reflectarray Antenna (ISARA) mission.

The primary purpose of CUMULOS is to be a staring sensor and having three cameras, which can capture one frame from each camera simultaneously with the other two cameras 
to scan the required region. These two sensors, CUMULOS and R3, can integrate the more complex payloads on CubeSats to conduct remote sensing research.

In the part of remote sensing research activities, Vala et al. [41] proposed a camera system for cloud monitoring designed to be incorporated in remote sensing satellites. Using this system, clouds' presence can easily be detected, which is useful for applications like weather, oceanography, disaster assessment and geology, etc. The satellites' captured images get contaminated and transmit false information to the Earth stations due to clouds. The proposed cloud monitoring system is miniature and consumes less power. This system behaves like a secondary camera system that can identify clouds' presence and a corresponding decision will be sent to the primary camera to turn ON or OFF. The hardware unit of the detector head assembly and the camera electronics are incorporated to form a cloud monitoring system. The proposed cloud detection algorithm is implemented on INSAT satellites, NOAA GOES satellites. The system's future development task is to identify the thickness of the clouds and its black box approach is shown in Figure 29.

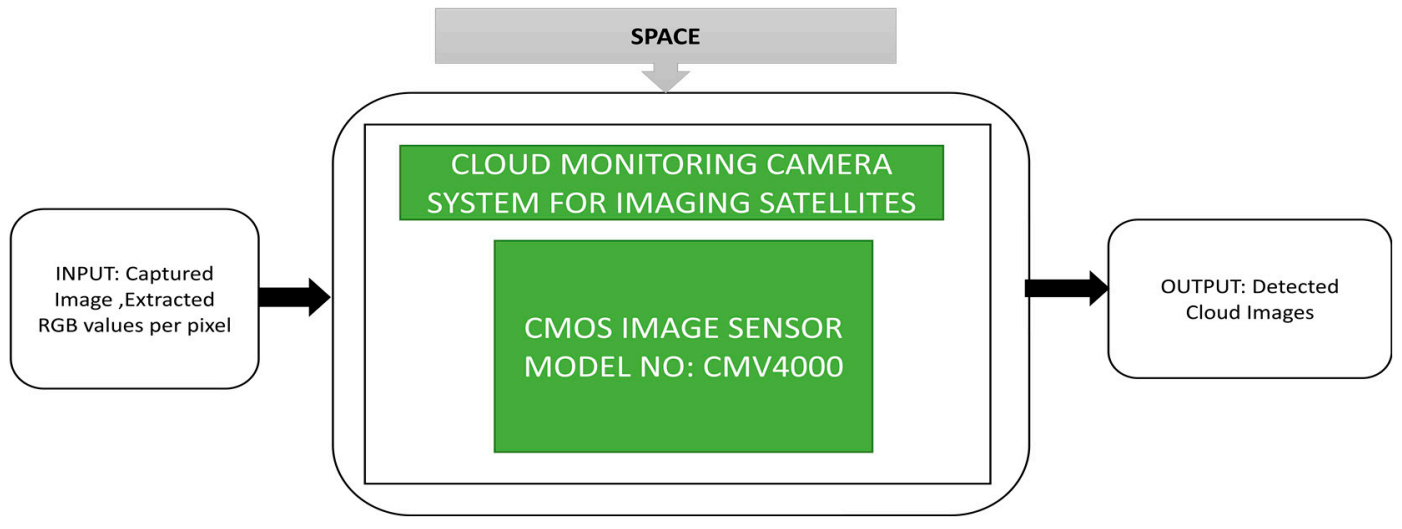

Figure 29. Cloud monitoring camera system for imaging satellites- INSAT satellite and NOAA GOES satellite.

CMOS image sensors have become one of the essential blocks in space applications like remote sensing satellites, Earth observation satellites, rovers, etc. Earlier CCDs were used as imagers for these applications due to their low noise and high image quality functions but CCDs consume more power, are big in size, and not tolerant of what? Due to these drawbacks of CCDs, CIS are chosen instead. Kim et al. [42] proposed a pixel design that can enhance CISs'capacity without including additional photocharge in the predefined pixel area. A known fact is that the damage caused by the radiation effect on CIS will be decreased by shrinking the CMOS technology to deep submicron size and reducing the thickness of the oxide layer.

Two tests are performed to find the radiation tolerance for the proposed imager, the displacement damage dose (DDD) test and total ionizing dose (TID) test. DDD test is conducted with metal shielding to protect the remaining electrical components (first image) and without metal shield (second image) as shown in Figure 30b. A total ionizing dose (TID) test is conducted by usinggamma radiation as a radiation source, as shown in Figure $30 \mathrm{c}, \mathrm{d}$. By performing these two tests, this imager is radiation tolerant and suitable for use in space applications.

It is essential to know the information of asteroids, including their shape, surface composition, internal structure and surface morphology, to know their origin and evolution. Around 14 types of asteroids have been found and assessed so far by nanospacecraft such as CubeSats. Nanospacecraft can characterize and provide detailed information about various asteroids in a mini time frame by sending many spacecraft at a time to monitor numerous targets. 


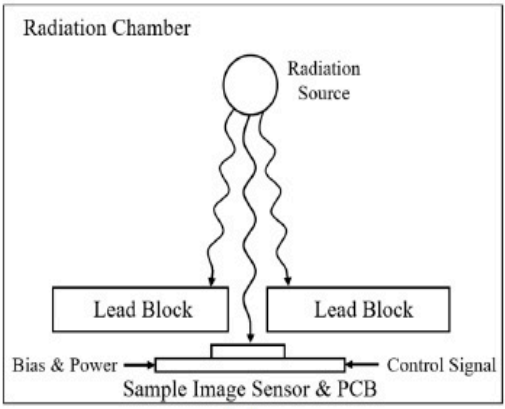

(a)

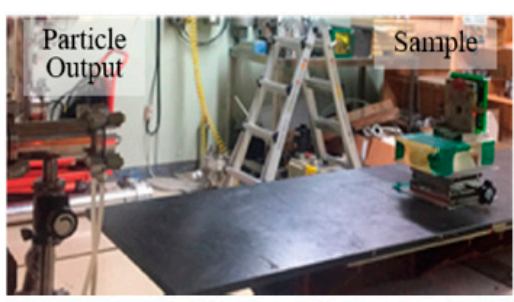

(b)

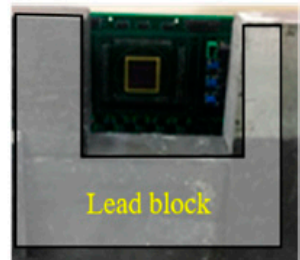

(c)

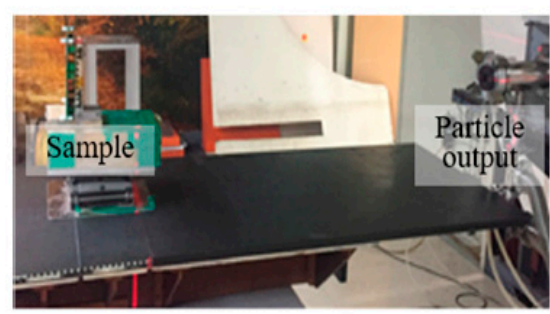

Figure 30. (a) Radiation test setup block diagram; (b) Displacement damage dose test with metal shielding(first image) and without metal shield (second image); (c) Total ionizing dose test setup front view; (d) Total ionizing dose setup with radiation source [42].

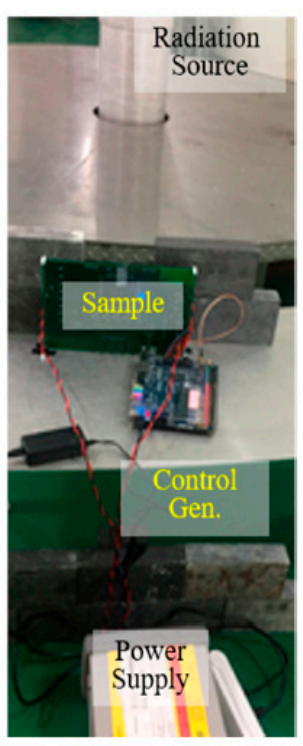

(d)

Pajusalu et al. [43] developed a prototype and simulated a nanospacecraft virtually to characterize the asteroids. The multi-asteroid touring (MAT) nanospacecraft mission is an example of this approach. The corresponding design used in MAT nanospacecraft missions and the prototype captured images of the moon, and its black box approach is shown in Figure 31.

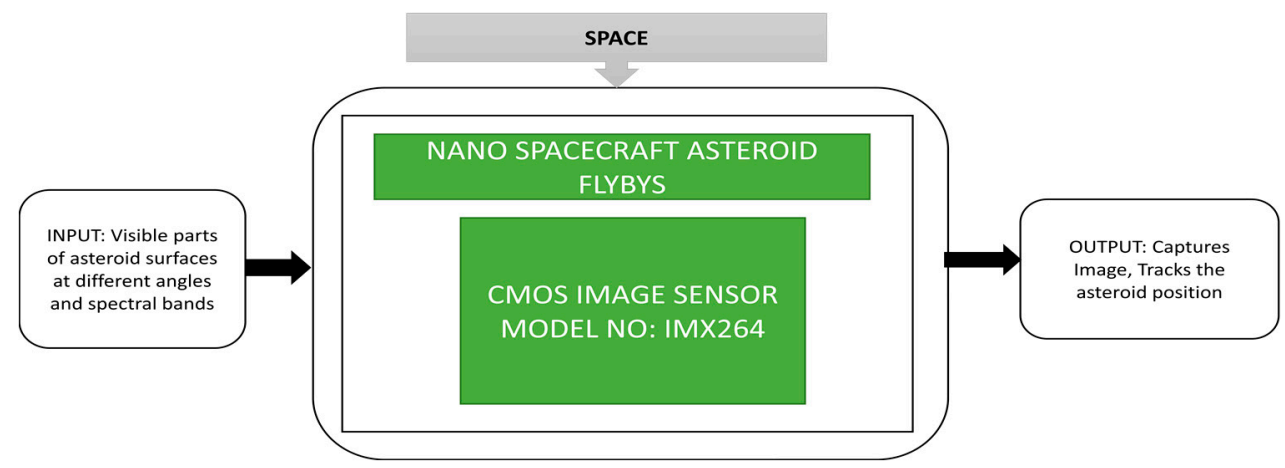

Figure 31. Spacecraft for Multi Asteroid Touring (MAT) mission.

Due to daily human activities, there is a gradual increase in the concentration levels of greenhouse gases in the atmosphere, leading to drastic changes in the climate. This climate change is influencing the water content levels on Earth in most of the areas very severely. Due to this effect, the productivity of crops is also affected to a great extent. CubeSats [44] could be used to monitor the levels of greenhouse gases. Jallad et al. [4] described the MeznSat, a 3U CubeSat which carries a shortwave infrared (SWIR) microspectrometer as its primary payload to detect the greenhouse gas levels in the atmosphere. The two familiar greenhouse gases that exist in the atmosphere are carbon dioxide and methane. Due to its high heat absorption property, methane cannot stay longer in the atmosphere than carbon dioxide.

To find the nutrient concentrations in the sea of the Arabian Gulf, algal boom occurrences could be predicted by using a CMOS image sensor-based RGB camera in the shortwave infrared region. The primary objective of this MeznSat is to identify the carbon 
dioxide and methane levels in the atmosphere with the help of a shortwave infrared region spectrometer. This satellite's primary payload is the Argus2000 shortwave infrared spectrometer, and the secondary payload is a CIS-based RGB camera shown in Figure 32a. The mechanical structure of Mezn Sat, which is a $3 \mathrm{U}$ CubeSat with its solar cell distribution representation, is shown in Figure 32c,d. This satellite was initially scheduled to launch in March 2020 and launched successfully on 28 September 2020 from Russia, and it is a UAE's most prestigious national project. Small satellites and CubeSats can increase the availability of astrophysics measurements, but an astrophysical demonstration is needed to realize their potential before any measurements.
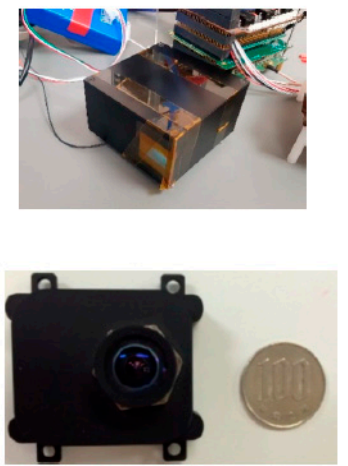

(a)
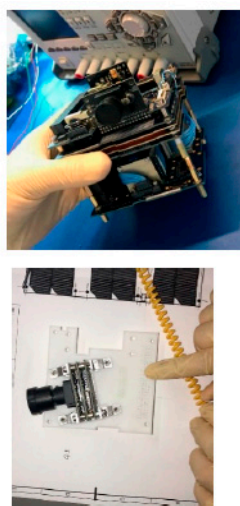

(b)

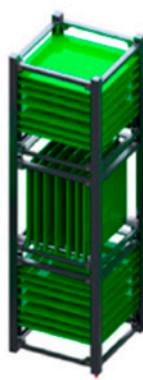

$3 U$

(c)

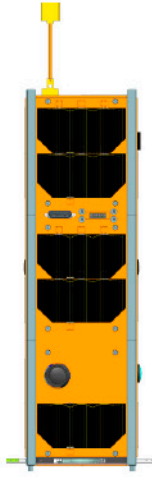

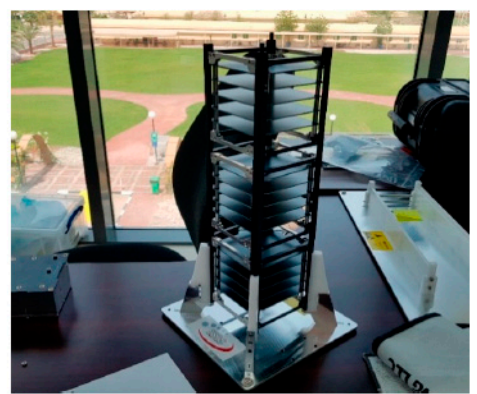

(d)

Figure 32. (a) Argus2000 Spectrometer with CIS based RGB camera; (b) Attitude determination and control subsystem (Upper Image) and star tracker (lower Image); (c) 3U CubeSat platform and its solar cell distribution; (d) Mechanical structure of MeznSat [44].

Knapp et al. [45] explained the 6U CubeSat space telescope-Arcsecond Space Telescope Enabling Research In Astrophysics shortly called ASTERIA demonstration for high precision photometry. The mission of ASTERIA is to launch a demo to measure the distance between small planets that are present around nearby stars. Various missions were conducted in space to determine the space parameters like types of stars, planet evolution, and magnitude of stars. The Transiting Exoplanet Survey Satellite (TESS) mission [46] was launched in 2018, did a survey about the total sky for a series of observations for one month. The Planetary Transits and Oscillations of stars (PLATO) mission [47] with 26 small telescopes are used to analyze the sky's bright stars. ASTERIA is a small space telescope with higher photometric precision than available ground telescopes and a device to perform bright star observations. The payload of ASTERIA is a combination of an optical telescope and electronics. The CMOS image sensor acts as an imager in the optical telescope. The layout and assembled ASTERIA satellite and its black box approach is shown in Figure 33.

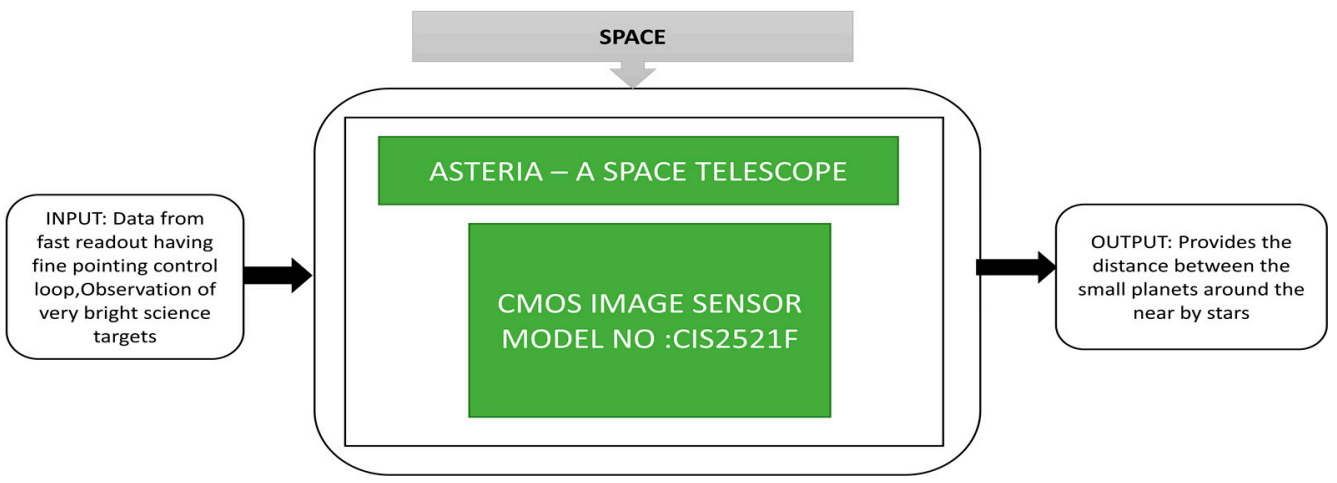

Figure 33. Arcsecond Space Telescope Enabling Research in Astrophysics (ASTERIA). 
A CMOS image sensor is used as a science detector in ASTERIA due to its fast readout mechanism compared to CCDs. Another additional feature of ASTERIA is the fault protection system, which protects the battery from undervoltage situations. It can also reach the target star, start observation, and come back to an attitude of Sun point within 43 min after observation with the help of a backup battery.

\subsection{Military CIS Applications}

CIS is becoming crucial in military applications like aerial surveillance, battlefiled monitoring, target recognition, missile detection, etc. Wireless aerial image transmission is one of the key modules in war zones. Zhang et al. [48] developed a portable wireless aerial image transmission system by using an unmanned air vehicle, the CIS OV9653, to capture images, work using an advanced blackfin BF531/533 digital signal processing system, and its architecture with operation is explained terms of its black box approach in Figure 34 .

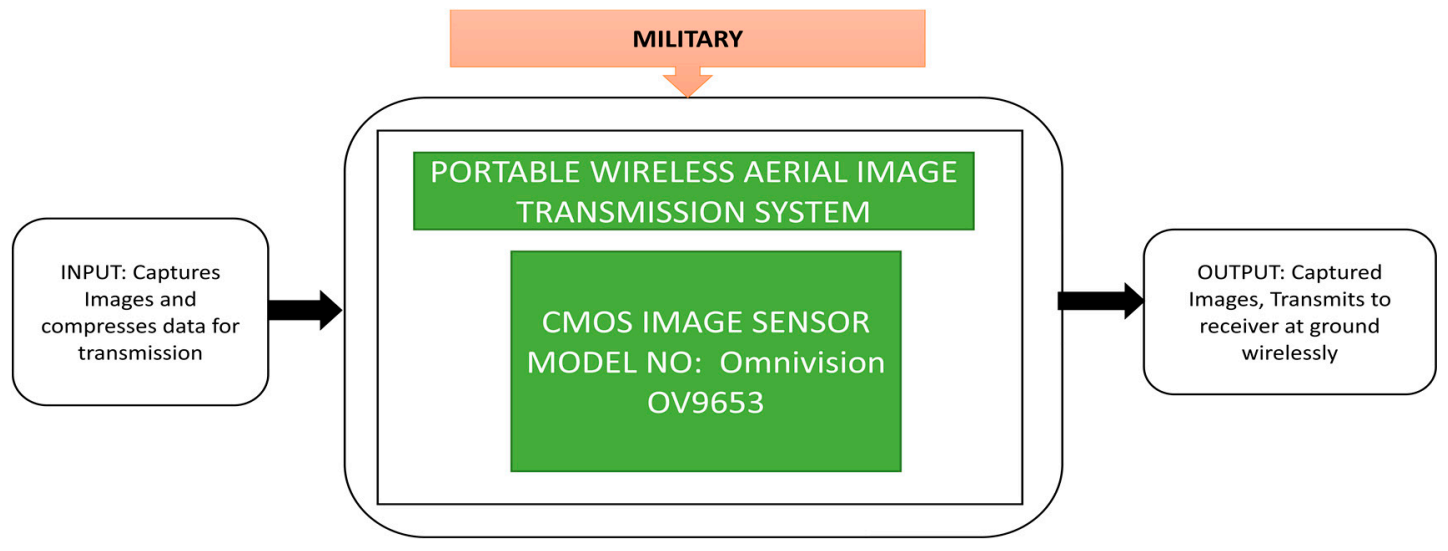

Figure 34. Portable wireless aerial image transmission system.

The BF531 will compress CIS's images, whereas the BF533 receives and monitors the images on the ground by sampling the data in real-time. It is well suited for field reconnaissance applications and can also monitor bad weather situations. Due to the high demand for aerial surveillance in the military field, especially to know aerial information, including soldier counts, equipment, weapons, etc., unmanned aerial vehicles play a pivotal role in fulfilling military needs aerial surveillance. Blumenau et al. [5] developed an intelligent portable aerial surveillance system termed IPASS, which black box approach concept shown in Figure 35. IPASS is a portable, reliable, user-friendly system that can send aerial images to a ground control station using wireless transmission. IPASS also met the designed specifications, including a maximum reachable height of 100 feet, surviving a fall from 30 feet, and has a wide vision, location detection, image, and local wireless data transmission capability up to 200 feet.

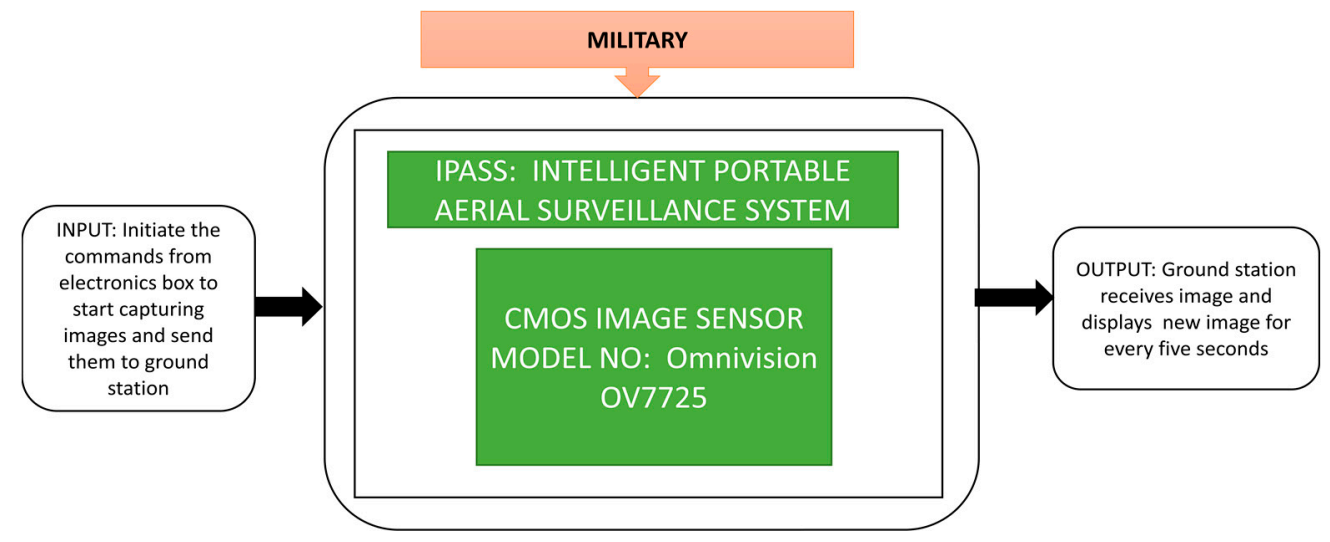

Figure 35. Intelligent Portable Aerial Surveillance System (IPASS). 
This system helps fighters in the battle zone collect visual information and transmit the data wirelessly within 120 feet. Odour et al. [49] explained the low-cost multispectral camera developed by Banpil Photonics (city, state abbrev if USA, country). It is a highperformance short wave infrared imaging camera, most suitable for military applications like threat detection during the day and at night, in all weather conditions. Its black box approach is explained in Figure 36.

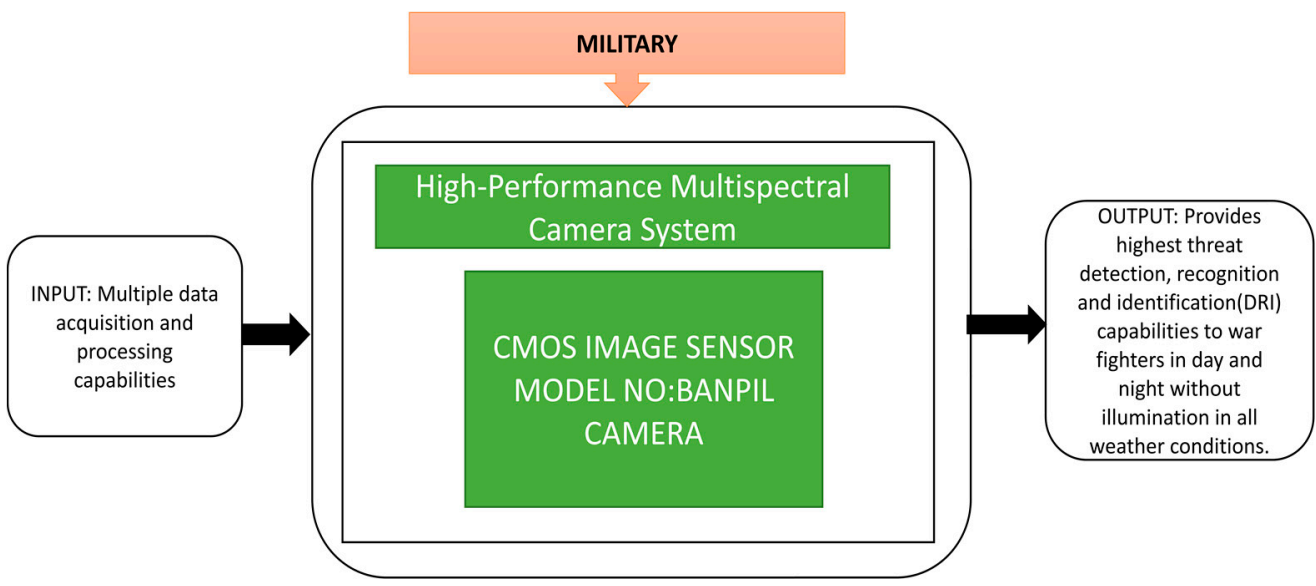

Figure 36. Banpil Multi-Spectral Camera.

This camera had multiple functionalities like data acquisition and processing to support soldiers in threat detection, recognition, and identification (DRI) capabilities to ensure situational awareness. Thermal imaging is also an advanced technology used in defense applications. It can detect an object with thermal contrast from the background without using natural light or other light sources. Kurum et al. [50] proposed a technique similar to binning, a well-known function used for mid-wave infrared imaging detectors to get a good signal-to-noise ratio. An experiment was conducted on a missile system to evaluate the pixel pitch and resolution, which need many of these imagers. Resolution and signal to noise ratio are the two parameters to calculate the detection range and recognition and identification of targets. These two factors are compared in binning enabled and disabled conditions of image acquisition. The most critical missile and weapon detection parameter recognizes the target and avoids damage to the neighboring ships or civilian ships across the sea. A higher resolution is required to identify the target among them. The captured images of a civilian ship and no warships were detected, and the object is identified with the target in red color on a background in blue using the thermal imager. Its black box approach is shown in Figure 37.

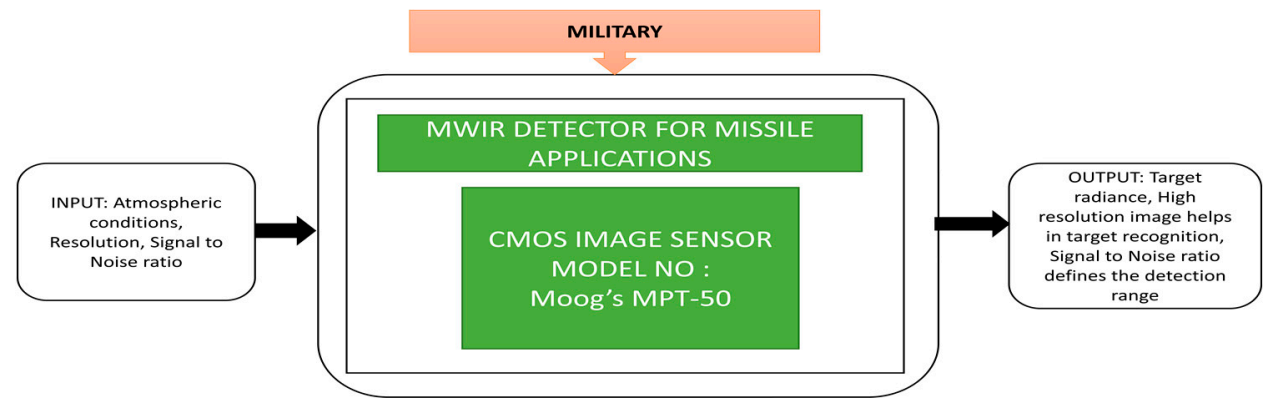

Figure 37. Mid wave infrared imaging detector for missile applications.

The study of ballistics is the background for live-fire testing in the army training areas to maintain safety measures. The study of ballistics is classified into the interior, exterior, and terminal ballistics. Interior ballistics refers to the gun's barrel's internal process when the gun is ignited and till the bullets come out of the barrel. Exterior ballistics describes how 
the bullet left the barrel with an angle, where terminal ballistics explains what happens after the bullet hits the target. D'Aries et al. [51] presented their experimental results produced from conducting X-ray imaging of rifle bullets fired out of a gun barrel using high frame rate cameras. In this experiment, a CIS is coupled to a scintillator to act as an X-ray image detector. This X-ray imaging has three instruments: a scintillator screen, an X-ray source, and an X-ray detector. Continuous frames of three images were captured at 10,000 frames per second when the bullet is fired from the barrel, and a grenade launcher was also captured while it was firing. The black box approach is shown in Figure 38.

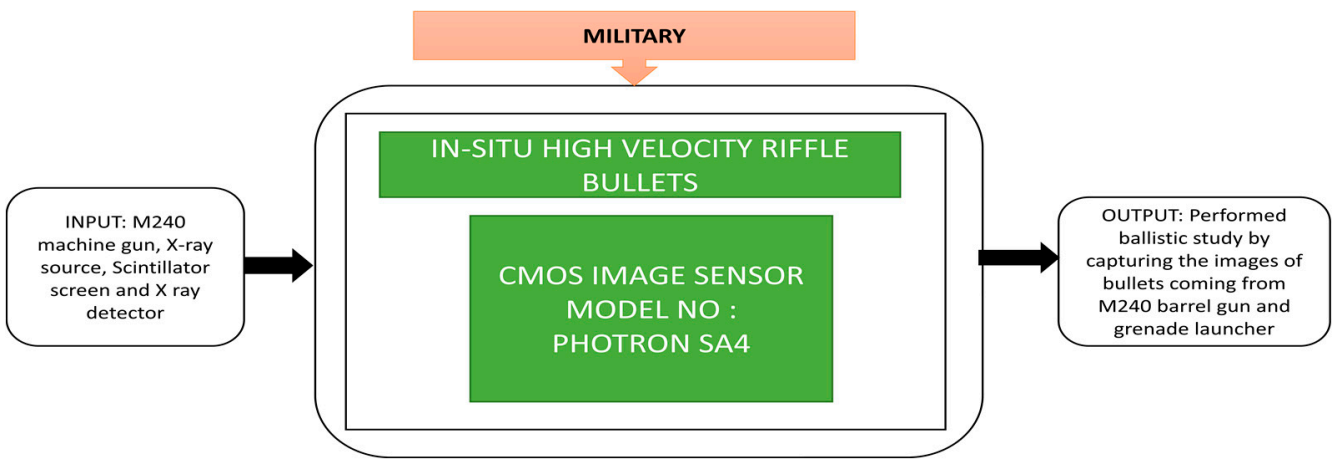

Figure 38. Ballistics experiment using X-ray imaging with a grenade launcher and M240 barrel gun.

Using wireless sensor networks, wireless image sensor networks are made to provide the observation area's visual data. Pandey et al. [52] proposed a technique to implement a wireless image sensor that can be used for monitoring and surveillance. This wireless image sensor network has a CMOS image sensor integrated with internal processing and transmission modules. Some of the nodes are connected to radiofrequency, and the rest are connected for Bluetooth transmission.

The black box approach of a visual sensor node prototype is shown in Figure 39. An image is captured using the CMOS image sensor incorporated in the node internally, and the images are compressed with different ratios to transmit through the XBee communication module.

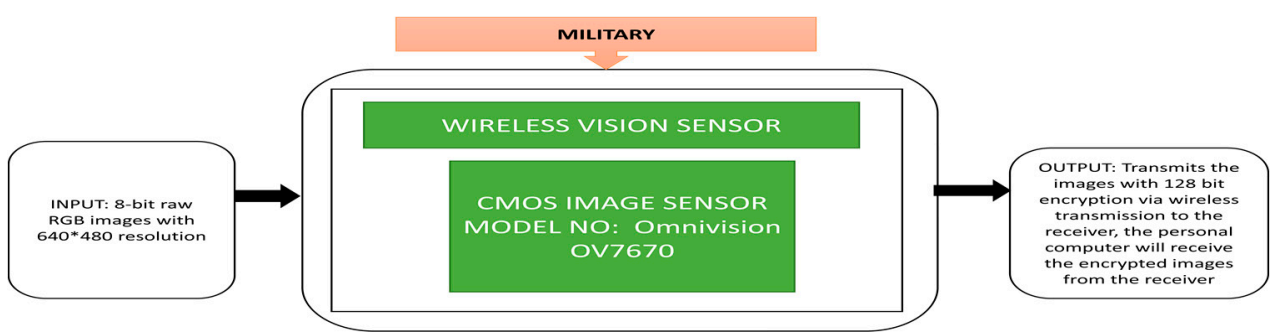

Figure 39. Wireless vision sensor.

Involvement of unmanned aerial vehicles is increasing rapidly in military warfare, surveillance and weather monitoring applications, etc. The US Army has employed a huge collection of categories of unmanned aerial vehicles in the field to perform Intelligence, Surveillance and Reconnaissance (ISR) missions to do search and rescue operations. However, these systems need to land for recharging or refueling. Johnson et al. [53] developed a CARMA similar to the real-time indoor autonomous vehicle test environment (RAVEN), a prototype developed by MIT [54]. CARMA refers to Catch and Release Manipulation Architecture, which enhances the RAVEN applications. This prototype is used to influence manipulation in industries to recharge and capture unmanned aerial vehicles (UAV) which are busy in monitoring and surveillance purposes. The main feature of this system is to capture, track, and charge the unmanned aerial vehicles. The components and architecture of CARMA and its black box approach are shown in Figure 40. 


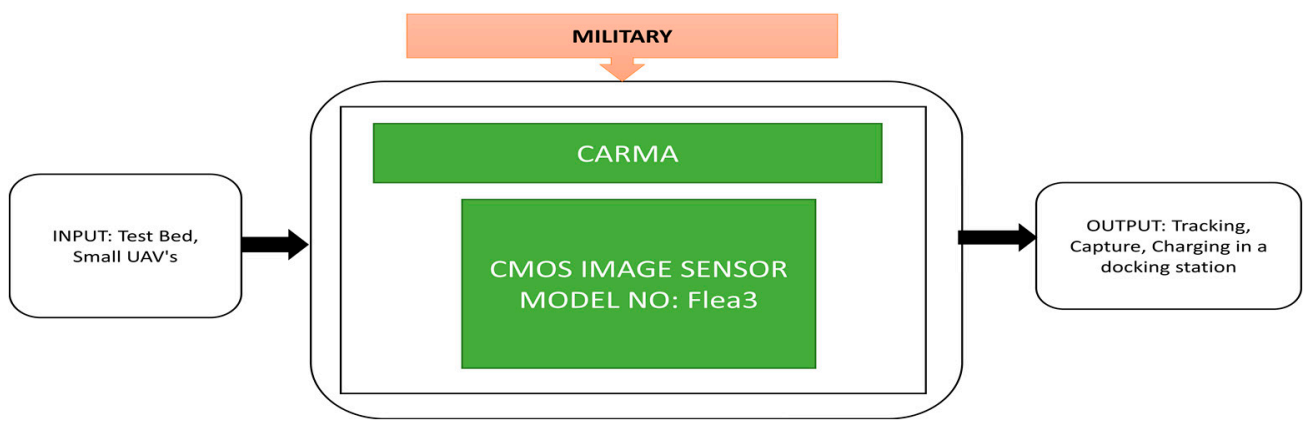

Figure 40. Concept of Catch and Release Manipulation Architecture (CARMA).

In the process of refueling or recharging the UAVs, the testbed on the ground vehicle will hold the UAVs in the docking station until they get charged. The undercarriage will be a platform for the UAV to safely dock it into the docking station and charging ports will charge the UAV.

Placing bombs onto vehicles and causing them to explode to create a human loss is one of the major terrorist threats every nation faces now. To overcome this issue, many detection mechanisms are being developed. Majeed et al. [55] proposed an Under-vehicle Inspection System (UVIS) to detect bombs under the vehicles in real-time to monitor and save the people from terrorist attacks. The prominent features of UVIS include good image clarity to show the size, location, shape of the bomb, a clear view of the bomb under the vehicle, a fast processing time to perform the detection task, comfortability, and security. This system can grab the license plate information to detect the suspect or stolen cars and other vehicles using license plate recognition technology. The on-demand screen will help check under the car for bomb inspection and the driver image camera will capture the drivers' image to match the crime database. The entire Under Vehicle Inspection System (UVIS) is shown in Figure 41.

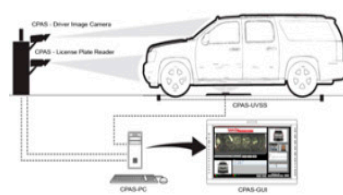

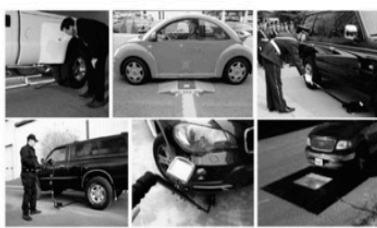

(b)

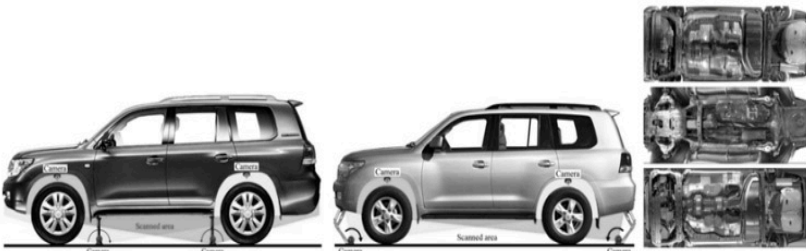

(c)

Figure 41. (a) Under Vehicle Inspection System (UVIS); (b) Real-time Inspection; (c) Under view for bomb Inspection [55].

Katz et al. [56] developed an architecture involving the CMOS SPAD imager for gun muzzle flash detection. This imager can detect the fast optical and weak signals in very high illumination environmental conditions. Its unique quality is the detection of gunshots quickly in combat fields. The detection system architecture, imager mounted on board, and complete field experiment setup is done in daylight conditions where we will get high illumination. Its black box approach is shown in Figure 42.

During the past decade, the usage of small airplanes is increasing and providing comfort to human living. The misuse of these aircraft causes illegal activities and affects human privacy and country security. Different techniques are introduced to overcome this problem, like net catching, laser weapon shooting and electronic interference, etc. The reconnaissance balloon at high altitude technique is a familiar aerial reconnaissance aircraft with detecting components that are terminated by blasting the balloon tail knot. In this aircraft, laser weapons cannot shoot the target effectively by penetrating the balloon due to surface penetration capability. If the laser continuously targets the balloon's knot, then it will burn the balloon and thereby destroy the target.

Hong et al. [57] proposed an identification method to detect the balloon knot in various illumination conditions and destroy the target. A CMOS image sensor-based detector is 
used to capture the balloon images under different light conditions with various contrast and blurred values. Field experiments were conducted to identify the knots of white and black balloons under low, high contrast, and medium and heavy blurred lightning conditions. The corresponding black box approach shown in the cited paper is presented in Figure 43.

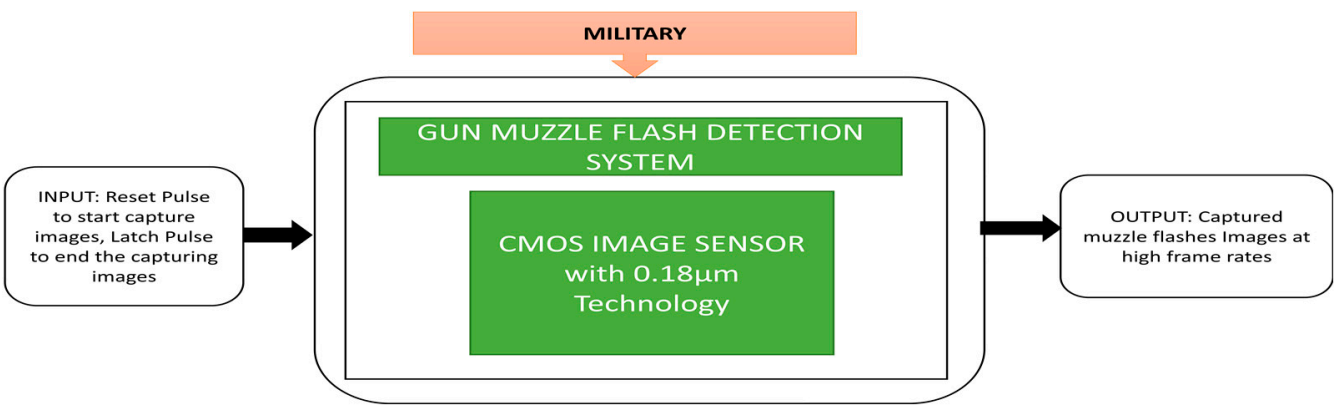

Figure 42. Gun muzzle flash detection system.

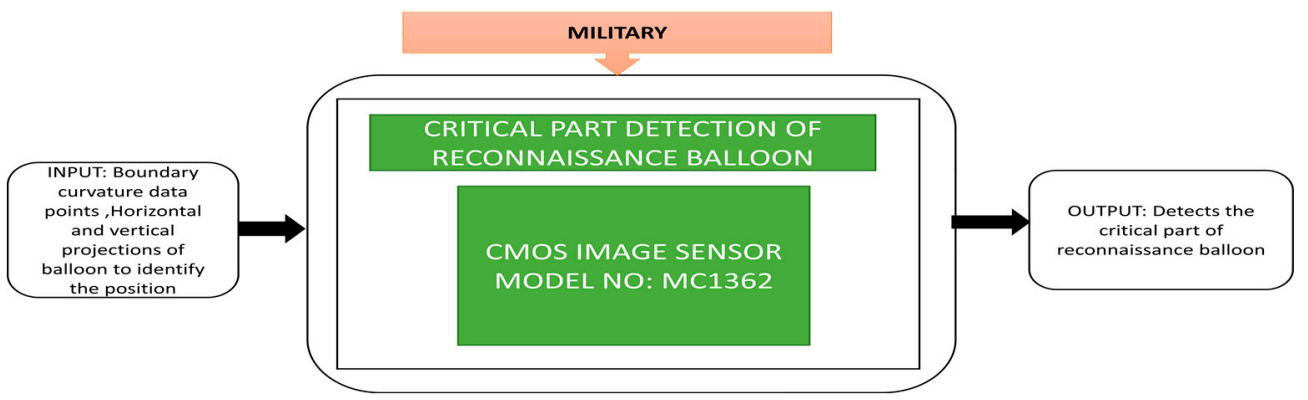

Figure 43. Reconnaissance balloon critical part detection.

\subsection{Automotive CIS Applications}

Over the past 20 years, intelligent transport systems (ITS) have grabbed colossal recognition. CMOS image sensors' role in automotive applications focuses on applications inside the vehicle, vehicle to vehicle, and vehicle and traffic. Inside the vehicle, applications involve capturing real-time images and detecting lanes by the vehicle's motion. Different lane identification algorithms were proposed from the beginning of the 20th century. Many improvements have been reviewed to meet weather requirements and traffic conditions. The other CMOS imagers developed have mostly focused on high resolution, dynamic range, and noise reduction instead of integrability in compact applications. Hsiao et al. [58] developed a CIS which can capture an image and detect traffic lanes simultaneously. This lane detection system can detect lane markers and capture road images in real-time in different weather conditions, as shown in Figure 44.

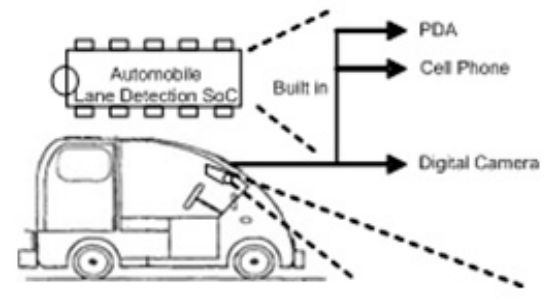

(a)

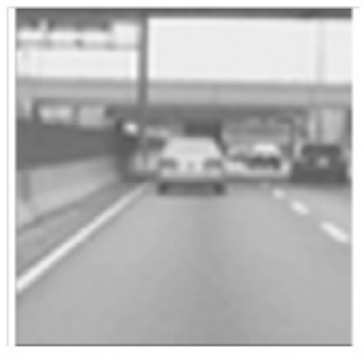

(b)

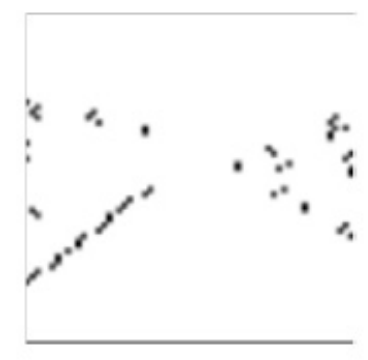

(c)

Figure 44. (a) Automobile lane detection using CMOS image sensor; (b) Original captured image; (c) Image captured by CMOS imager [58]. 
Zhang et al. [59] proposed an on-screen display architecture and SPI interface on CIS used for automobile applications. The backup camera is equipped in the vehicle with the on-screen display embedded in a field-programmable gate array (FPGA) to detect the lanes with the black box approach explained in Figure 45. It achieves a dynamic range of $70 \mathrm{~dB}$.

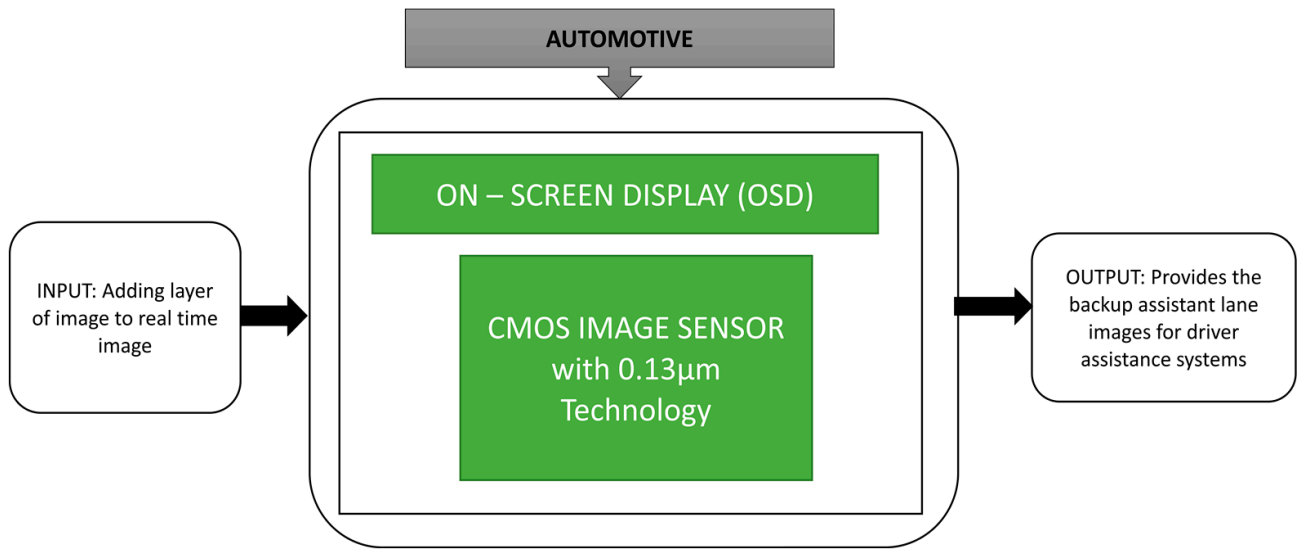

Figure 45. On Screen Display (OSD).

To meet one of the requirements in advanced driver assistance systems, which monitor the driver's emotions, Cao et al. [60] developed a time-resolved CIS for non-contact heart detection imager was tested under invisible light and enormous ambient light conditions. An NRI LED array is used to detect the $\mathrm{HbO}_{2}$, i.e., oxyhemoglobin, in human blood. The concentration of oxyhemoglobin is gradually changed with respect to the heartbeat.

The driver's facial skin will be an object at a distance of $50 \mathrm{~cm}$ from the NIR light source. Cardiac rhythm output was recorded under different bright conditions. The black box approach is represented in Figure 46.

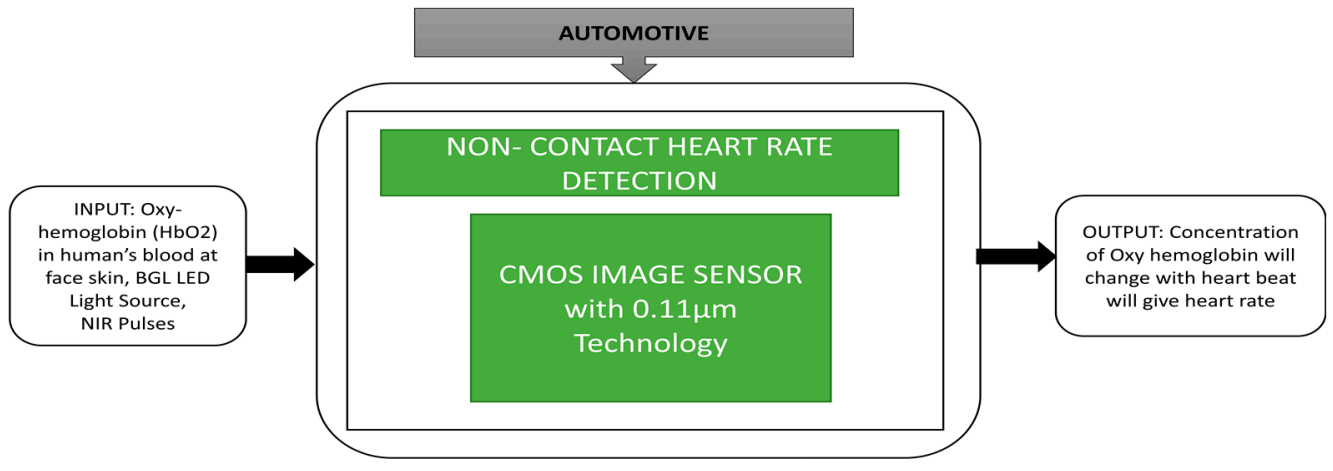

Figure 46. Non-contact heart rate detection of driver during driving the vehicle in motion.

Vehicle to vehicle communication is a prominent automotive application. Turturici et al. [2] implemented an affordable and flexible embedded system for fish eye automotive cameras in real-time. Fisheye cameras [61] suffer from tangential and radial distortion. It is very crucial to provide accurate vision to the driver by adjusting the captured video of the fisheye camera. Its black-box approach is explained in Figure 47.

Visible light communication utilizes low power light-emitting diodes (LEDs) to provide light and broadcast data in vehicle-to-vehicle communication, a rapidly growing application in intelligent transport systems (ITS).

Yamazato et al. [62] introduced two types of communications in automobile applications, namely vehicle-to-interface (V2I) and vehicle-to-vehicle (V2V). A V2I-VLC system field trial was conducted to observe the efficiency under real driving conditions. The black box approach is explained in Figure 48. An LED array is placed on the ground horizontally, and a high-speed camera is mounted on the vehicle's dashboard. The vehicle is driven 
at a speed of $30 \mathrm{kmph}$, and the communication distance ranges between $30 \mathrm{~m}$ and $70 \mathrm{~m}$. It has been observed that audio signals are received very clearly up to $45 \mathrm{~m}$ distance with no error. In this field trial, there are two vehicles named the lead vehicle and the following vehicle. The lead vehicle has two LED transmitters, a front view camera to take front view images, and a control unit. LED transmitters are attached to the left side, and the right side of the rear window sends an optical signal with 40 degrees inclination. The control unit collects the vehicle's internal data, such as speed, vehicle id, and data (encoded packets) from LED transmitters. This collected data will be sent to the following vehicle.

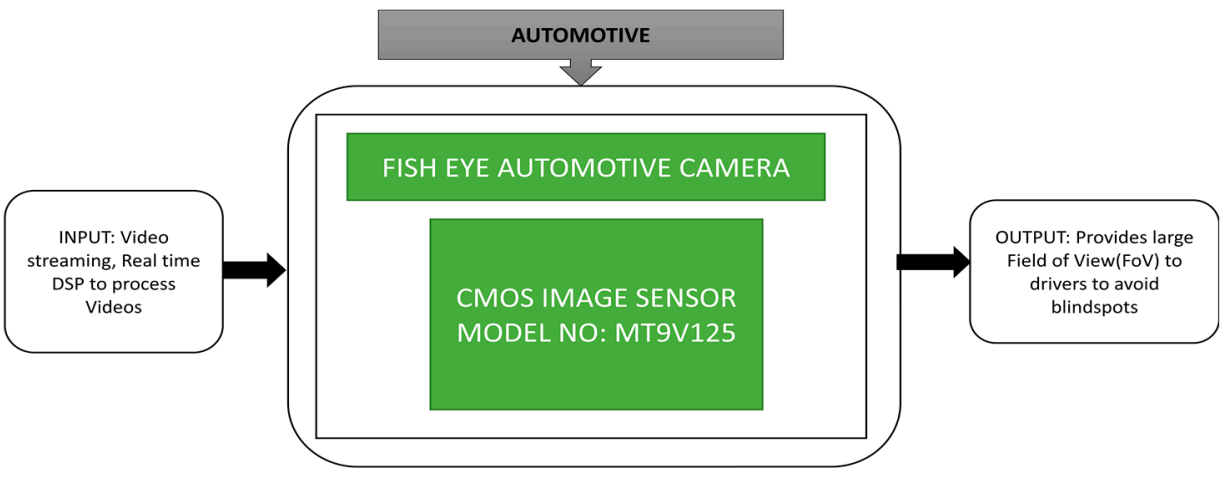

Figure 47. Fish eye automotive camera for blind spot detection.

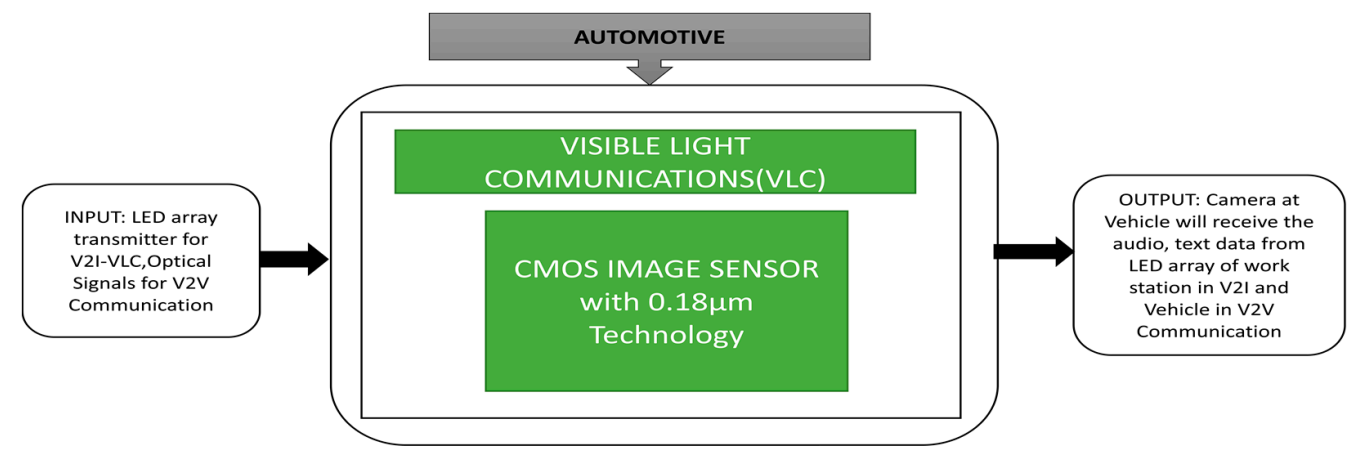

Figure 48. Visible Light Communication (VLC) in two modes of operation namely vehicle to interface (V2I)-VLC using an LED traffic light and a vehicle to vehicle-based VLC System (V2V)-VLC using LED brake lights.

Takai et al. [63] developed an image sensor-based optical wireless communication system using a LED as transmitter and a camera as a receiver with a data rate of $10 \mathrm{Mbps}$ per pixel to send colored videos and vehicle internal data, including speed, the status of brakes, etc. It can also communicate with moving vehicles as an additional feature. The black box approach is shown in Figure 49.

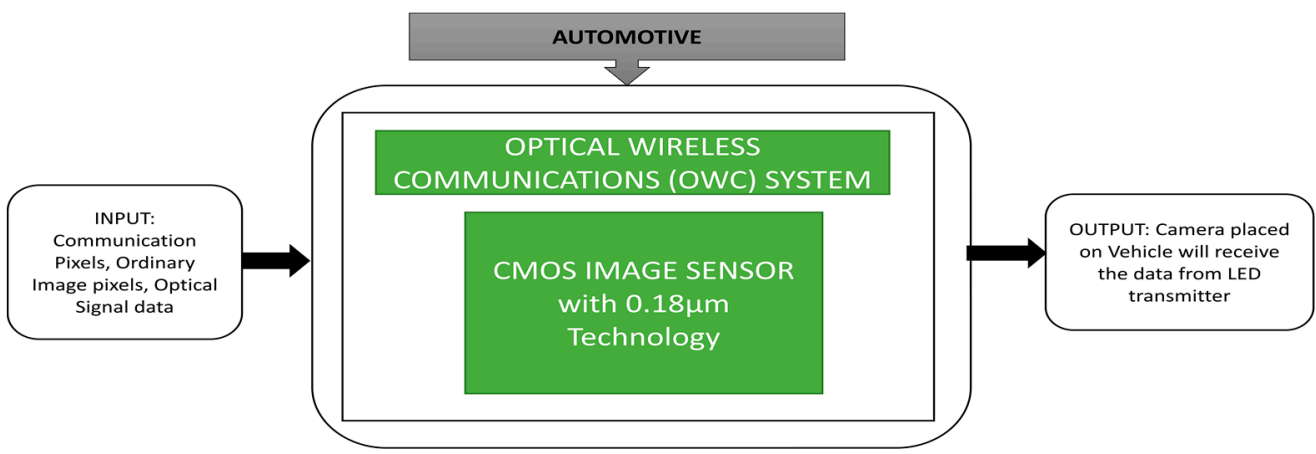

Figure 49. Source identification using image sensor based optical wireless communication system. 
Bronzi et al. [64] developed an optical 3D ranging camera using a CMOS Single-Photon Avalanche Diode (SPAD) imager, one of the more prominent drives assistance applications in the automotive sector.

A 3D ranging camera is mounted on the car to conduct some test trials for two cases: a stable object case and an object in motion case. In the case of a stable object, the images were captured in a parking lot in which the illumination power is enough to make the light hit the target up to a distance of $40 \mathrm{~m}$. We can easily observe the individual's distance at seven meters and pillars with different ranges and the second case, where the object is in motion when the car is taking a left. The corresponding black box approach is indicated in Figure 50.

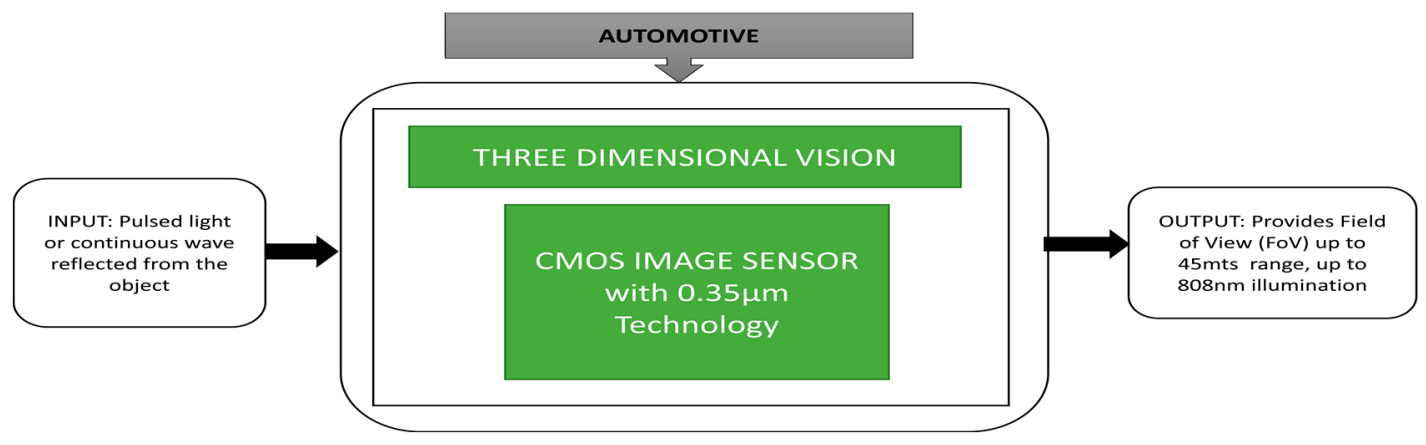

Figure 50. 3D ranging CMOS SPAD camera for advanced driver assistance systems.

Kwon et al. [65] developed a camera-based blind spot detection system for IoT based smart connected cars and replaced the existing radar-based blind-spot detection system.

Three cameras were mounted on the vehicle on the left side, right side, and rear view positions. Continuous monitoring of the three cameras' images can be seen in the display to avoid the blind spots while the vehicle is in motion.

Spivak et al. [66] developed a CIS for night vision systems. Its black box approach in shown in Figure 51. It is able to synchronize with an outside light source to extract information. This image sensor provides a wide dynamic range with a minimum of $92 \mathrm{~dB}$. Diaz et al. [67] proposed a unique method to detect traffic lights during day and night environmental conditions and measure distance. This function will be a part of the driver assistance system, which can identify traffic lights with more accuracy from a distance of 10 to $115 \mathrm{~m}$. A demonstration of this method happened on public roads in 2013 in Italy, as shown in Figure 52. Mu et al. [68] proposed an algorithm to identify car path tracking using an open MV in which a CIS is incorporated to recognize the white and black trajectory paths. With this algorithm, a car can select the track of the path instantaneously.

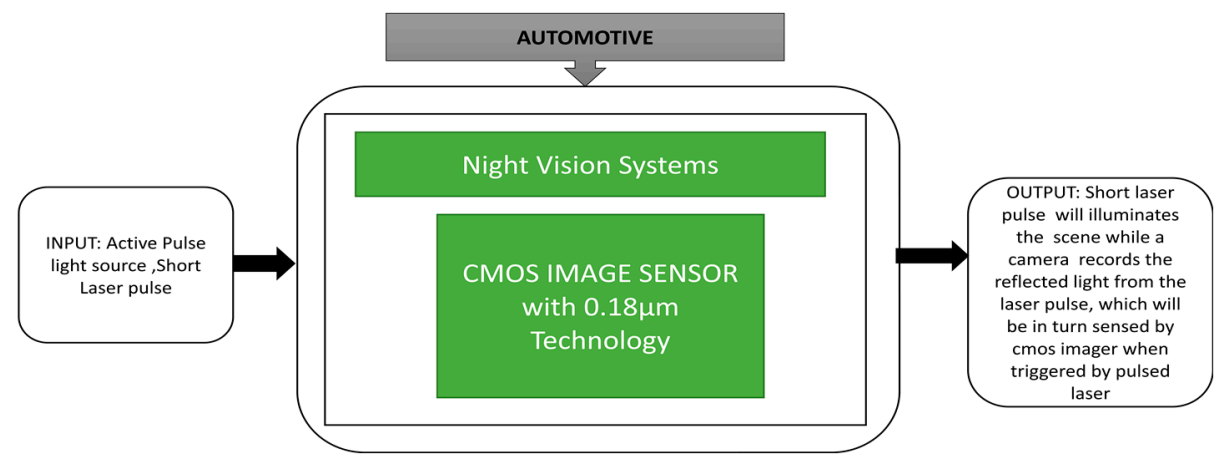

Figure 51. Night vision systems. 


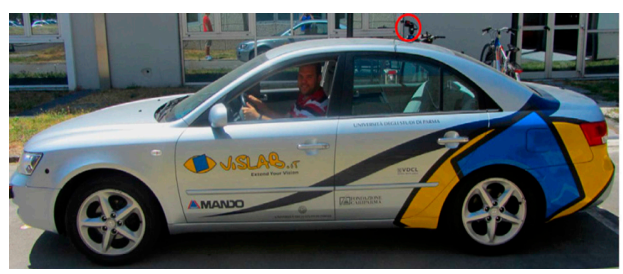

(a)
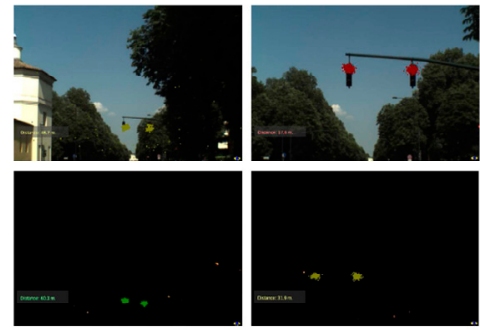

(b)

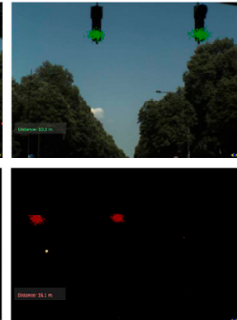

Figure 52. (a) Traffic light detection with camera; (b) Detection of traffic lights during day and night scenarios. Adapted permission from [67], Elsevier 2015.

\subsection{WSN (Wireless Sensor Networks) CIS Applications}

Wireless sensor networks play a prominent role in our day-to-day lives by advancing healthcare systems, home automation, temperature control, and environmental monitoring. Among the various WSN applications, those depending on light sensors or temperature sensors produce small data samples. However, changes in WSN technology make these sensors collect more information and integrate CIS to gather huge visible information of the target with less power consumption.

Bagree et al. [6] developed a wireless image sensor network to monitor a tiger's movements called "TigerCENSE." It monitors previously unseen movements remotely and can be accessible in some dangerous places to watch the tiger's behavior. A passive infrared sensor will activate this camera, and an integrated CMOS image sensor will capture the images that will be stored in the storage device. To avoid any white flashes that could disturbs the animal's movements, an infrared flash is used to illuminate the scene during nighttime. The stored images will be sent to a remote station through a radio transceiver and will be transfered to the server database using internet link nodes. A solar panel is used to charge wireless nodes' batteries to prevent frequent physical visits for battery changes. Tigers are usually differentiated by the stripe patterns on their body. No two tigers will have similar patterns. These patterns can help the researchers tigerCENSE to know their origin, presence, activity cycles, size of home ranges and behavior. The camera node will provide the tiger's path and capture the tiger's images using the infrared flash during the night. The prototype of the WSN was used for wildlife monitoring in field trials, and its black box approach is explained in Figure 53. TigerCENSE is reliable, nonintrusive, portable, consumes less power, and can be used in environments where humans cannot enter.

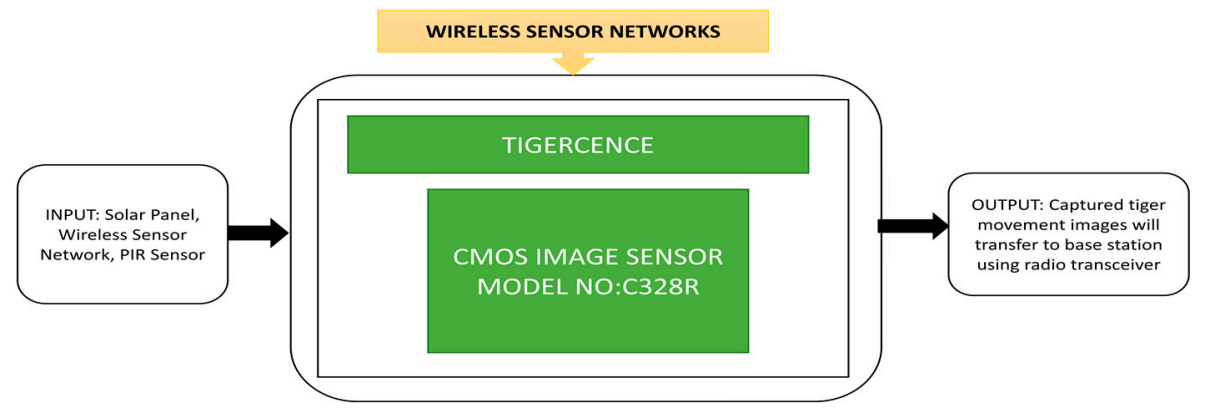

Figure 53. TigerCENSE.

Zhao et al. [69] developed a moving object detection and localization CIS that can be integrated into wireless sensor nodes to perform robotic vision surveillance functions. This sensor will automatically change to the region of interest mode by obtaining the target object's size and the position to capture the target image. The image sensor will capture grey level images during its normal intensity mode and capture the relevant temporal difference modes. A localization process will be applied to the temporal difference and 
automatically switch to the region of interest mode to capture the moving objects. Its black box approach is shown in Figure 54.

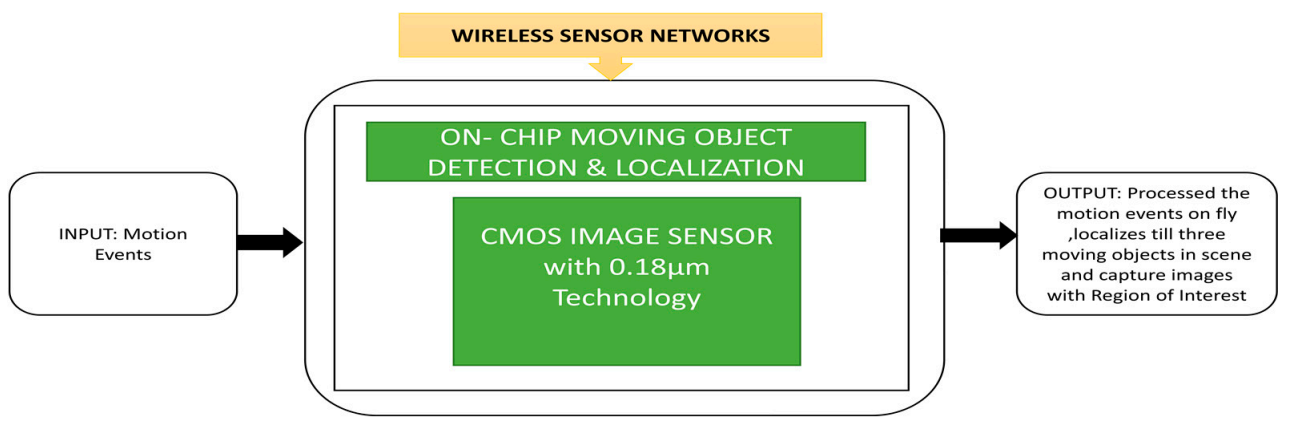

Figure 54. On chip moving object detection and localization using CMOS image sensor.

Jelicic et al. [70] developed MasliNET, a multimodal environmental system that uses a wireless sensor network to monitor pests in olive groves. It also contains a self-energy harvesting unit to provide sufficient power for the system. MasliNET was deployed on a real olive farm. The image sensor can capture trapped flies' images and send them to a server at a remote station. The system's unique function is the lowest power consumption over a long period and the fact it works under the worst climatic conditions. Its black box approach is shown in Figure 55.

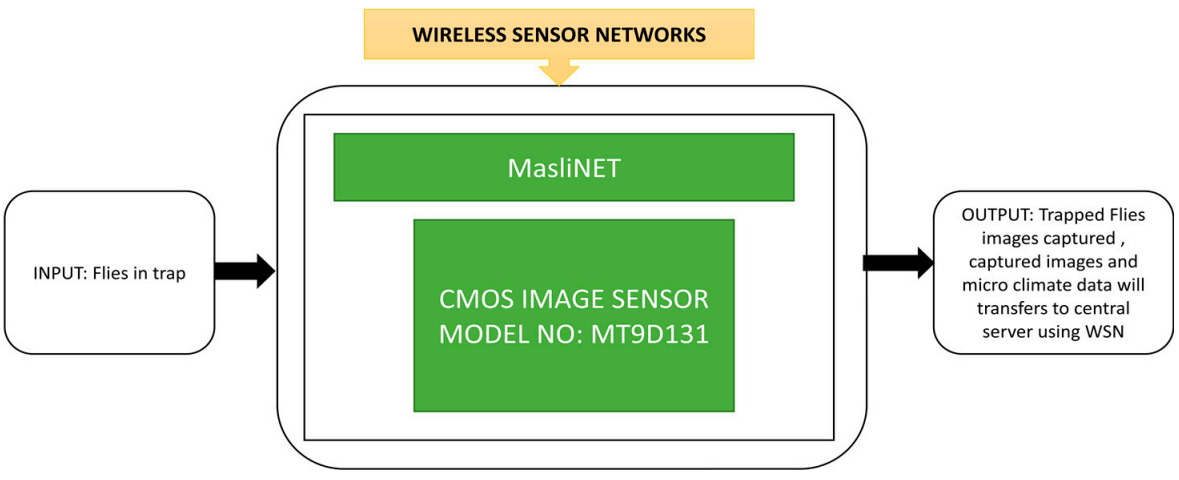

Figure 55. MasliNET-olive grove monitoring system using WSN.

Luo et al. [71] developed a wireless sensor network to observe the Heihe river basin's eco-hydrological process in which spatial dense parameters and material characteristics will be obtained. It is challenging to deploy networks in river basin areas due to the harsh climatic conditions and rough terrain. Suitable safety measures have been taken to deploy the network system at the basin area and gather the data to transfer via different repeaters to a remote station. Its operation with repeaters and its black box approach are clearly explained in Figure 56.

Controlling the pest population in agriculture is quite crucial in the case of forest and farm protection. Human labor is needed to perform frequent time-based trap surveys in agriculture fields, which consumes more time, labor, and is costly, especially for large fields or forest areas.

Lopez et al. [72] proposed an image sensor-based autonomous monitoring system which captures the trap images and sends them with timing label to a remote station. This system can cover huge areas while consuming significantly little power. An efficient pest controlling technique is to keep these pest traps uniformly distributed over the specified target area to be controlled. This trap system is focused on controlling the red palm weevil pest, which severely affects palm fields across the world. Wireless image sensors are connected to traps placed in fields from a wireless image sensor network. An aerial view of trap deployment over a specific area is shown in Figure 57. 


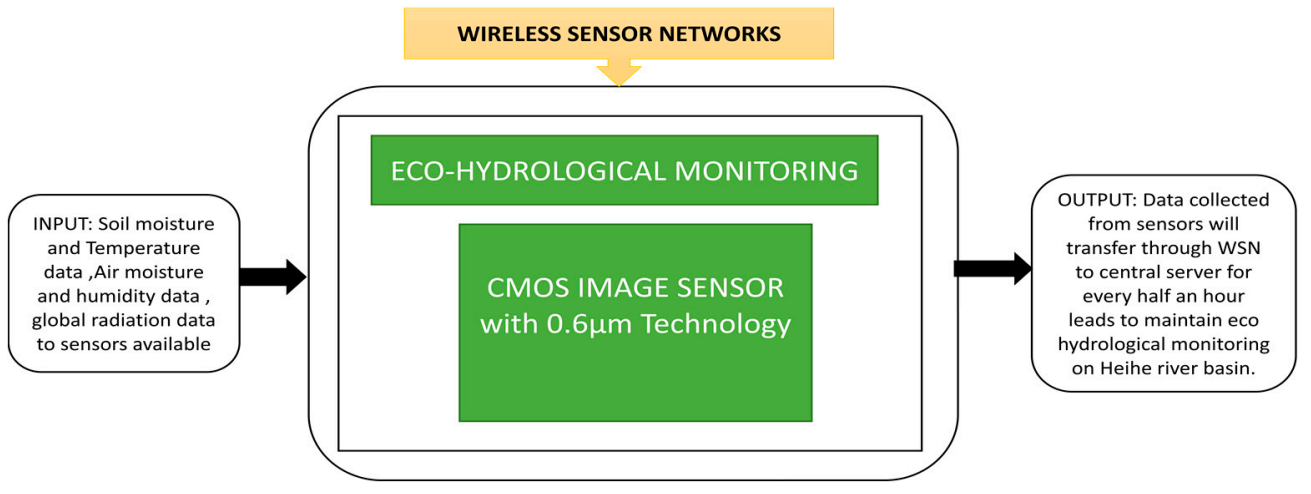

Figure 56. Eco-hydrological monitoring using WSN.

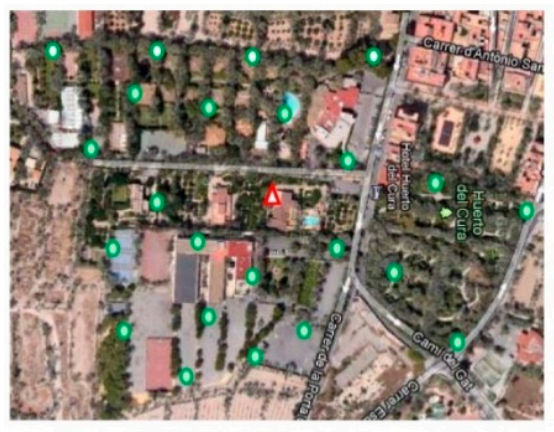

(a)

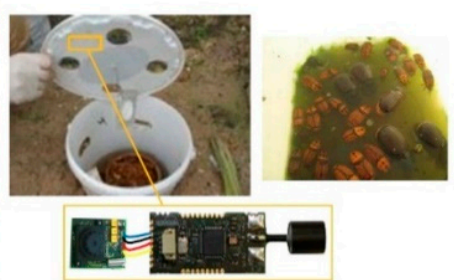

(b)

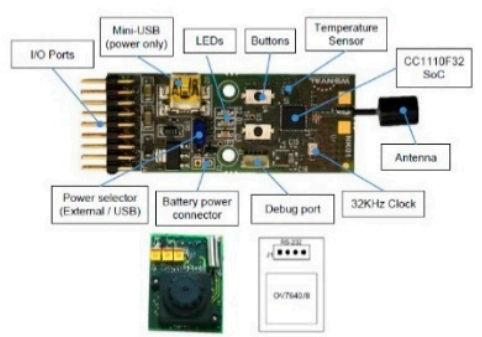

(c)

Figure 57. (a) Trap deployment aerial view; (b) Red Palm Weevil trap; (c) Image sensor used in trap [72].

During natural disasters like floods, the water can be contaminated contaminated entirely with highly viscous mud and objects, which prevents the instruments from measuring the water flow. To understand the ecological and hydrological process of rivers set of data containing the water stage, hydrograph discharge and velocity distribution are needed. Large Scale Particle Image Velocimetry (LSPIV) is a powerful and proficient method to measure river surface velocity to analyze the river's turbulence and flowing conditions in normal situations. Zhang et al. [73] developed a near-infrared imaging camera with internal preprocessing, image acquisition functions, as shown in Figure 58.

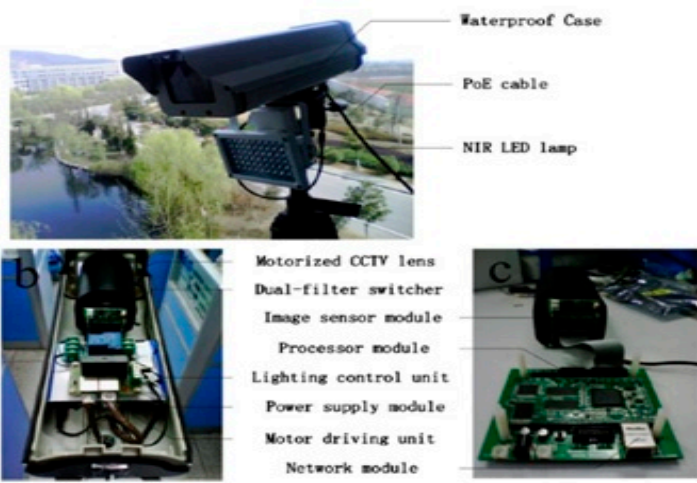

(a)

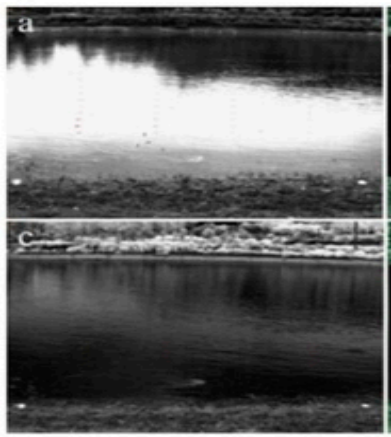

(b)

Figure 58. (a) Near Infra-Red (NIR) imaging camera with internal structure; (b) NIR captured images of river surface by applying LPSIV method in two different spectrum band with and without spatial high pass filtering. Adapted with permission from [73] Elsevier, 2013.

The NIR camera is placed at the LPSIV site to capture and process the images to improve the contrast between the target objects and the background and increase the peak SNR. Using spatial high pass filtering, the noise and the river's background will get suppressed efficiently, increasing the possibility of proper vectors in the immediate field 
flow. During the surveillance process in wireless sensor networks, cameras need to safely send the captured images to the remote station. Winkler et al. [74] proposed a unique feature in camera protection that involves security and privacy protection in an image sensing unit. Its black box approach is presented in Figure 59.

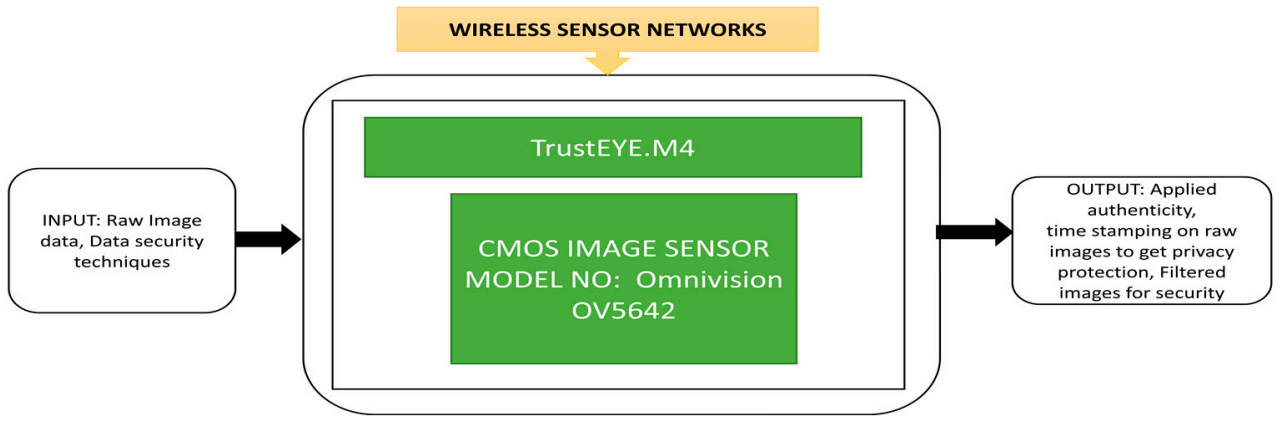

Figure 59. TrustEYE coupled with Raspberry Pi board having linux operating system.

Multimedia wireless sensor networks (MWSN) utilization is increasing in various applications, including wildlife monitoring, environmental monitoring, etc. In wildlife monitoring, zoologists have started using trap cameras to capture animals' images remotely without distributing them and trap cameras using MWSN to gather animals' visual information and habitual behavior. Camacho et al. [75] designed a trap camera and implemented an MWSN to monitor wildlife in Peru's Amazon rainforest. In this process, 25 trap cameras are installed and interconnected to form an MWSN at different forest locations. This monitoring experiment was conducted for six months by using 25 cameras in which only five cameras remained working at the end of the trial period. Its black box representation is presented in Figure 60.

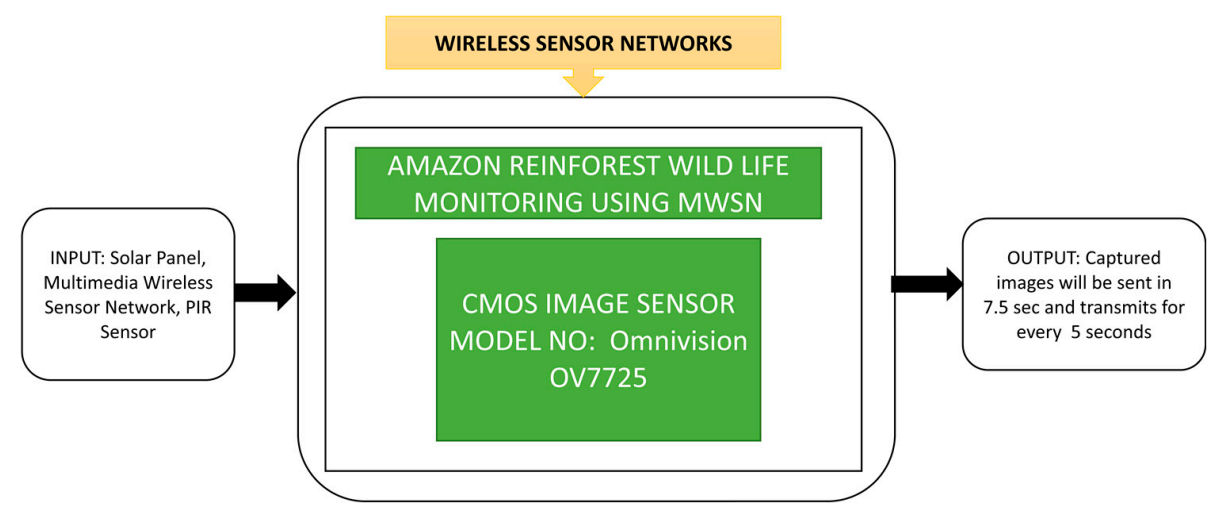

Figure 60. Amazon rainforest wildlife monitoring using multimedia wireless sensor networks (MWSN).

The author applied a cartoon filtering effect on the TrustEYE imaging sensing unit while capturing the images. This process will create privacy protection while detecting the region of interest. From the public transport and safety point of view, the outdoor lights along the roads, highways, parking lots, bicycle and pedestrian tracks must be switched on during nights and low light conditions. This in turn, causes light pollution, which must be calibrated. This calibration is needed, especially when Earth-observing satellites take images without blurring for better analysis. Fiorentini et al. [76] developed a sensor suite that can measure the intensity of luminance pollution of lights with a power spectral density function to detect the street lights' various lamp technologies. At present, air balloons and drones are using in measuring light emission instead of satellites. An instrumental suite called "MINLU" can be used to monitor the light pollution generated by outdoor lighting with drones and air balloons. Its blackbox approach is shown in Figure 61. 


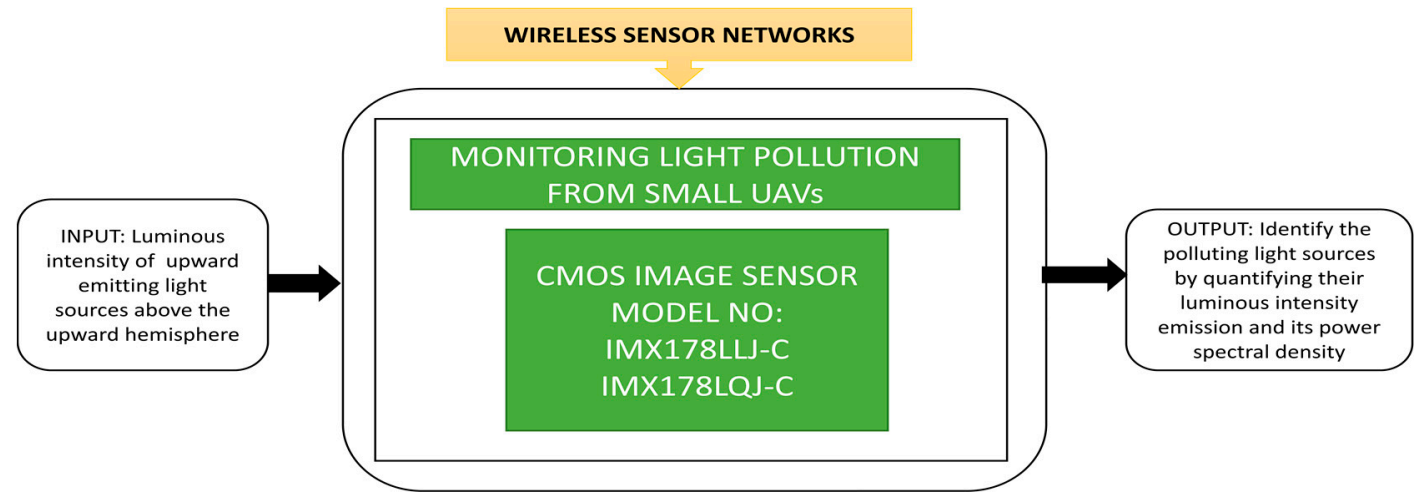

Figure 61. MINLU architecture for monitoring the light pollution from small UAV's.

The technical advancements in WSN applications is used in plant monitoring range from multispectral imaging to root phenotyping. A rhizotron imaging system is used to observe plant roots' growth statistics at farms, parks or public places.

It is a nondestructive, underground, repetitive process system, which is costly to perform. To overcome this, Rahman et al. [77] designed the SoilCam: a mini rhizotron multispectral imaging system with fully automated functions to perform onsite recording, monitor the plant root growth process, and allow phenotyping research of plants, as shown in Figure 62.

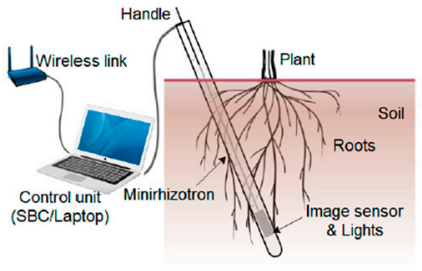

(a)

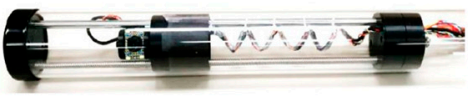

(b)

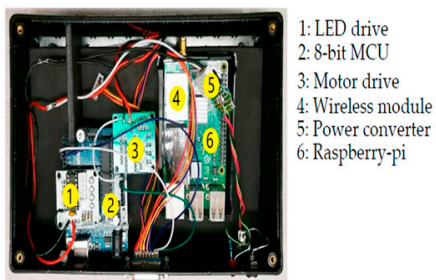

(d)

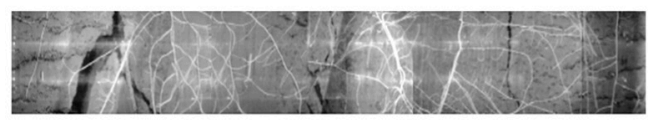

(e)

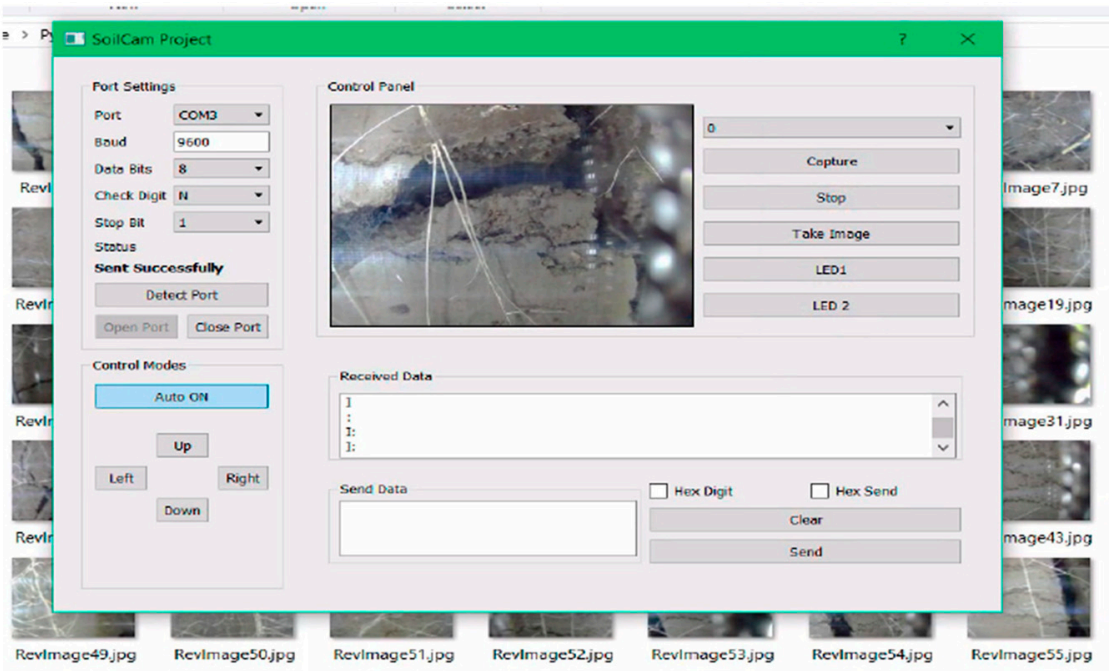

(c)

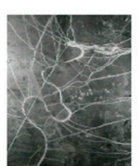

$850 \mathrm{rm}$

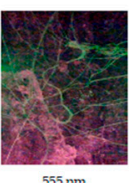

$555 \mathrm{rm}$

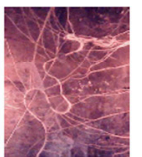

$700 \mathrm{~nm}$

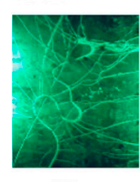

$525 \mathrm{~nm}$

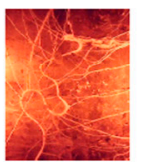

$630 \mathrm{~nm}$

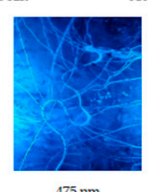

$475 \mathrm{rm}$

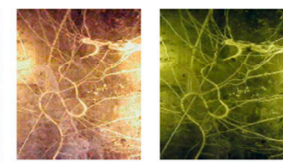

$610 \mathrm{~nm}$

$590 \mathrm{rm}$

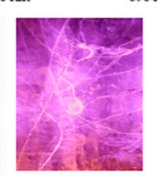

$395 \mathrm{rm}$

(f)

Figure 62. (a) Minirhizotron field experiment; (b) SoilCam; (c) Root and soil analyzer software; (d) Control box; (e) $360^{\circ}$ image captured by SoilCam of a Canola plant root; (f) Multispectral images captured by SoilCam [77]. 


\section{Design Characteristics}

\subsection{CMOS Technology}

According to Moore's law, the transistor number on CMOS chips will double every couple of years, which means the integrated device's speed will double. CMOS technology refers to the transistor gate length, which is reduced concerning the improvements and advancements occurring in the CMOS industry. Figure 63 presents the scaling of gate length to achieve double the density of transistors. The device's total scaling will be done by scaling both contacted poly pitch and minimum metal pitch to $70 \%$ to achieve a $50 \%$ reduction in area.

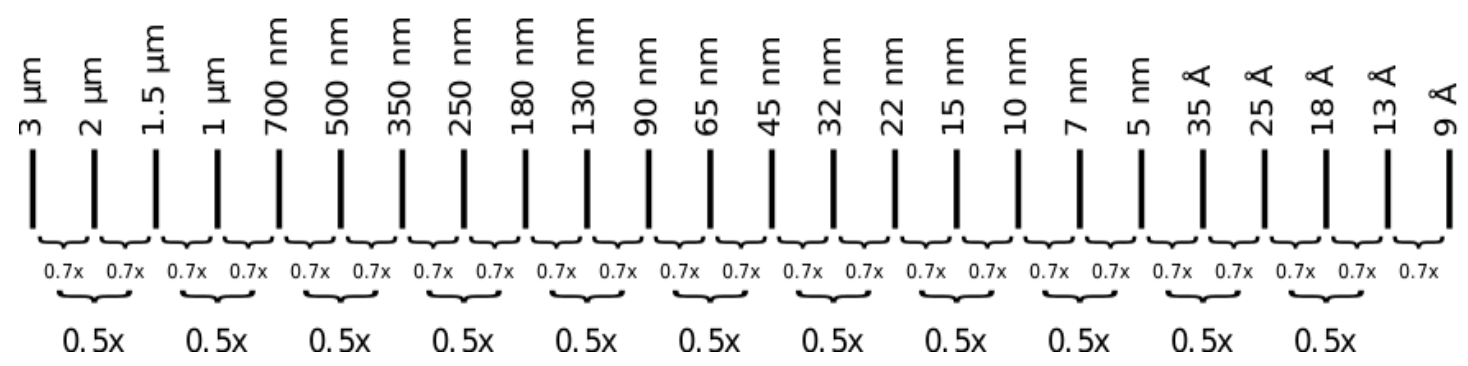

Figure 63. CMOS process technology variation [78].

\subsection{Resolution}

This is a parameter that represents the number of pixels in an array. This pixel count will form a two-dimensional pixel array in which more number of pixels in an array can give more quality images, as shown in Figure 64.
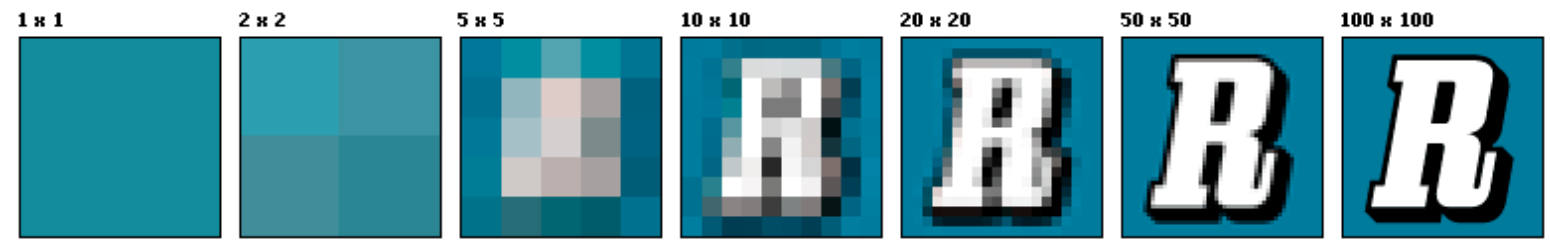

Figure 64. Various resolutions display [79].

\subsection{Dynamic Range}

The dynamic range is defined as the range of intensity of light in an image. The images produced by the image sensor with a high dynamic range are having light levels of low to high. The dynamic range's mathematical representation is the ratio of maximum saturated pixel output level to the level of noise in the dark [8]:

$$
\text { Dynamic Range }=20 \log \left(\frac{N_{\text {sat }}}{N_{\text {dark }}}\right)(\mathrm{dB})
$$

where $N_{\text {sat }}$ is the number of electrons collected by a pixel at saturation level, and this saturation level can be found by full well capacity. $N_{\text {dark }}$ is the number of electrons without illumination at the noise level.

\subsection{Frame Rate}

It is defined as the exposure time of the active pixel sensor and the frame readout time of the CMOS image sensor. If the exposure time decreases, then the frame rate increases, which means that the frame rate is inversely proportional to the exposure time. The units of the frame rate are frames per second ('fps') [8]. 


\subsection{Signal to Noise Ratio (SNR)}

SNR represents the pixel's fidelity in the CMOS image sensor, which is defined as a ratio of input signal power to the referred noise power at the input [8]:

$$
\mathrm{SNR}=10 \log _{10}\left(\frac{\left(i_{\text {ph }} t_{\text {int }}\right) 2}{q\left(i_{p h}+i_{\mathrm{dc}}\right) t_{\text {int }}+\sigma_{\text {read }} 2}\right) \mathrm{dB}
$$

where $i_{p h}$ is the photocurrent, $i_{d c}$ is the dark current $t_{\text {int }}$ is the integration time $\sigma_{\text {read }}$ is the read noise.

The CIS models are nothing but camera modules used as imagers in surveillance applications discussed field-wise in this paper. The design characteristics play a key role in choosing an appropriate CIS model for a particular surveillance application in fields like the military and space, etc. The mostly used CIS models in surveillance systems are the OV7670, OV7725, OV2640, OV2710, OV9653, and OV9655 manufactured by Omnivision (Santa Clara, CA, USA), the MT9V125, MT9V131 by ON Semiconductor (Phoenix, AZ, USA), the MC1362 of Mikotron GmbH (Unterschleissheim, Germany), and the CMV4000, CMV20000 of AMS (Plano, TX, USA). Hence, we tabulated CIS models' design characteristics termed as camera modules used in applications related to all the fields covered in our literature survey as shown in Table 1 and field-wise mapping of the CIS model, representing the CIS models used in each field as shown in Table 2. We have also tabulated the usage of CMOS image sensor models in applications by year in according with survey data having field-wise applications shown in Figure 65.

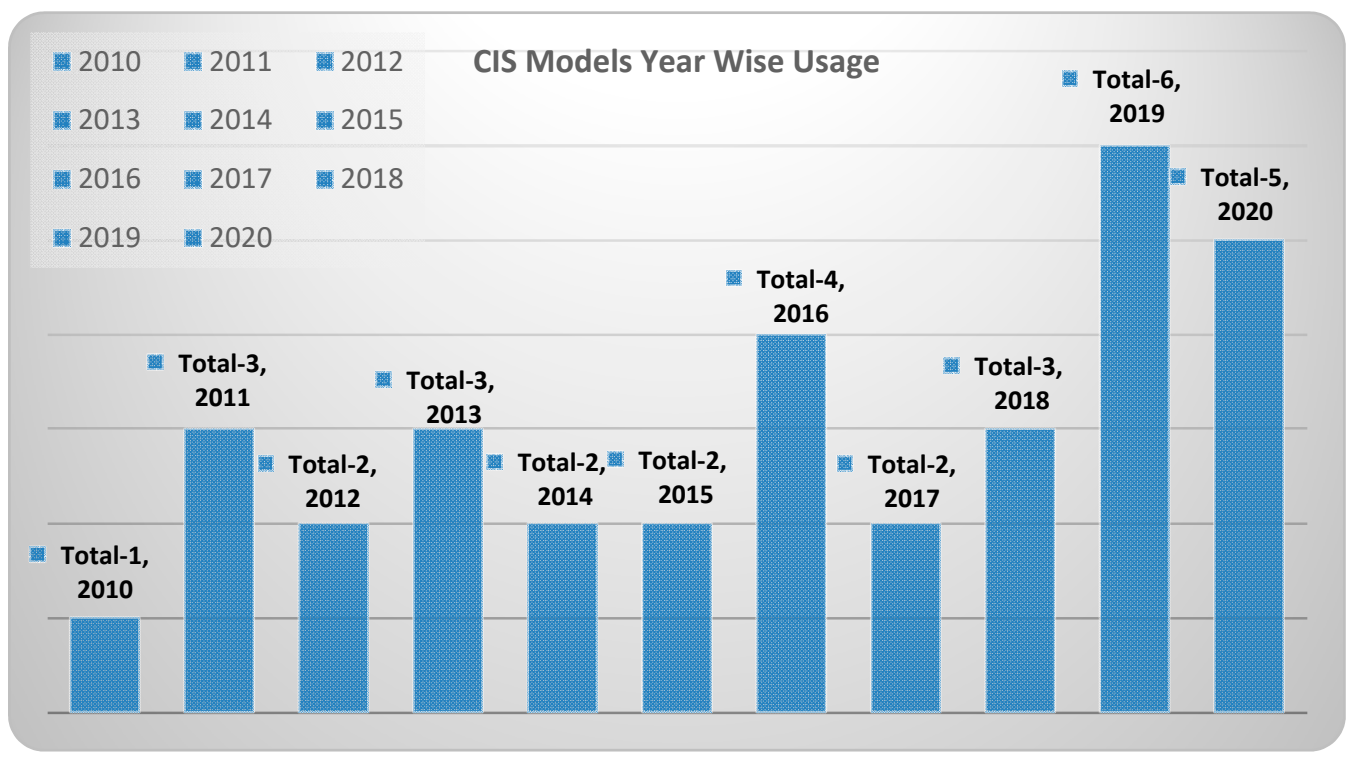

Figure 65. Year wise usage of CIS models according to survey data, where $\mathrm{x}$-axis represents years and y axis represents number of CIS models.

A lot of research and technological advancements are being made in surveillance systems. In security and biometric applications, gait recognition [80-83] has been introduced in surveillance systems to recognize the person by capturing his gestures while walking. This gait recognition technology can be operated from remote places using a server and also it can be applied to camera systems having low resolution to high resolution. The parameters like face and fingerprints datasets are not necessary to perform this gait recognition to identify people. 
Table 1. Design Characteristics of CMOS image sensors.

\begin{tabular}{|c|c|c|c|c|c|c|c|c|c|}
\hline S. No. & Year & Technology & Camera Module & Resolution & SNR (dB) & $\begin{array}{l}\text { Frame Rate } \\
\text { (fps) }\end{array}$ & $\begin{array}{l}\text { Dynamic } \\
\text { Range }(\mathrm{dB})\end{array}$ & Application Name/Target & Field \\
\hline 1 & 2009 & $0.35 \mu \mathrm{m}$ & $\mathrm{N} / \mathrm{A}$ & $64 \times 64$ & $\mathrm{~N} / \mathrm{A}$ & 10 & $\mathrm{~N} / \mathrm{A}$ & Built in lane Detection & Automotive \\
\hline 2 & 2011 & $0.18 \mu \mathrm{m}$ & $\mathrm{N} / \mathrm{A}$ & $128 \times 256$ & 51 & 60 & 98 & Night Vision Systems & Automotive \\
\hline 4 & 2013 & $0.18 \mu \mathrm{m}$ & $\mathrm{N} / \mathrm{A}$ & $642 \times 480$ & $\mathrm{~N} / \mathrm{A}$ & 30 & $\mathrm{~N} / \mathrm{A}$ & Optical Wireless Communication System & Automotive \\
\hline 5 & 2013 & $0.13 \mu \mathrm{m}$ & $\mathrm{N} / \mathrm{A}$ & $768 \times 576$ & 45 & $\mathrm{~N} / \mathrm{A}$ & 70 & On-Screen-Display (OSD) & Automotive \\
\hline 6 & 2014 & $0.18 \mu \mathrm{m}$ & $\mathrm{N} / \mathrm{A}$ & $642 \times 480$ & $\mathrm{~N} / \mathrm{A}$ & 60 & $\mathrm{~N} / \mathrm{A}$ & Visible Light Communication & Automotive \\
\hline 7 & 2015 & $\mathrm{~N} / \mathrm{A}$ & GUPPY-F036 C & $752 \times 480$ & $\mathrm{~N} / \mathrm{A}$ & 64 & $\mathrm{~N} / \mathrm{A}$ & Traffic light Detection & Automotive \\
\hline 9 & 2018 & $0.11 \mu \mathrm{m}$ & $\mathrm{N} / \mathrm{A}$ & $1280 \times 1024$ & $\mathrm{~N} / \mathrm{A}$ & 30 & $\mathrm{~N} / \mathrm{A}$ & Non Contact Heart rate Detection & Automotive \\
\hline 10 & 2018 & $\mathrm{~N} / \mathrm{A}$ & OV7725 & $640 \times 480$ & 50 & 60 & 60 & Intelligent Car Path Tracking & Automotive \\
\hline 11 & 2011 & $\mathrm{~N} / \mathrm{A}$ & OV6620 & $356 \times 292$ & $>48$ & 60 & $>72$ & Nilaparvata Lugens Monitoring System & IoT \\
\hline 12 & 2011 & $\mathrm{~N} / \mathrm{A}$ & OV7640 & $640 \times 480$ & 46 & 30 & 62 & Crop Monitoring System & IoT \\
\hline 13 & 2011 & $\mathrm{~N} / \mathrm{A}$ & Hercules Webcam & $1280 \times 960$ & $\mathrm{~N} / \mathrm{A}$ & 30 & $\mathrm{~N} / \mathrm{A}$ & Vine Yard Monitoring & IoT \\
\hline 14 & 2013 & $\mathrm{~N} / \mathrm{A}$ & VBM40 & $1280 \times 960$ & $\mathrm{~N} / \mathrm{A}$ & 30 & $\mathrm{~N} / \mathrm{A}$ & Human Monitoring System in Sea Transportation & IoT \\
\hline 15 & 2013 & $\mathrm{~N} / \mathrm{A}$ & OV9655 & $1280 \times 1024$ & $\mathrm{~N} / \mathrm{A}$ & 15 & $\mathrm{~N} / \mathrm{A}$ & Smart Camera Networks (SCN) & IoT \\
\hline 16 & 2016 & $0.18 \mu \mathrm{m}$ & $\mathrm{N} / \mathrm{A}$ & $64 \times 64$ & $\mathrm{~N} / \mathrm{A}$ & $\mathrm{N} / \mathrm{A}$ & 96.7 & $\begin{array}{l}\text { Smart Image Sensor with Multi Point Tracking } \\
\text { (MPT) }\end{array}$ & IoT \\
\hline 18 & 2018 & $\mathrm{~N} / \mathrm{A}$ & OV7670 & $640 \times 480$ & 40 & 30 & 52 & Precision Agriculture System Design & IoT \\
\hline 19 & 2019 & $\mathrm{~N} / \mathrm{A}$ & OV2640 & $1600 \times 1200$ & 40 & 15 & 50 & SMART HOME & IoT \\
\hline 20 & 2019 & $\mathrm{~N} / \mathrm{A}$ & SF3324-101 & $1928 \times 1208$ & $\mathrm{~N} / \mathrm{A}$ & $\mathrm{N} / \mathrm{A}$ & $\mathrm{N} / \mathrm{A}$ & CUbE & IoT \\
\hline 21 & 2009 & $\mathrm{~N} / \mathrm{A}$ & $\begin{array}{l}\text { Quickcam Pro } \\
\quad 9000\end{array}$ & $1600 \times 1200$ & $\mathrm{~N} / \mathrm{A}$ & 30 & $\mathrm{~N} / \mathrm{A}$ & Privacy preserving sensor for Person Detection & ISS \\
\hline 22 & 2010 & $0.18 \mu \mathrm{m}$ & $\mathrm{N} / \mathrm{A}$ & $64 \times 64$ & $\mathrm{~N} / \mathrm{A}$ & 30 & $\mathrm{~N} / \mathrm{A}$ & surveillance in low crowded environments & ISS \\
\hline 23 & 2015 & $\mathrm{~N} / \mathrm{A}$ & ucam-II & $128 \times 128$ & 44.2 & $\mathrm{~N} / \mathrm{A}$ & 51 & Visual surveillance and intrusion detection & ISS \\
\hline 24 & 2017 & $0.18 \mu \mathrm{m}$ & $\mathrm{N} / \mathrm{A}$ & $176 \times 144$ & 47 & 14 & 61.8 & Multi Resolution Mode & ISS \\
\hline 25 & 2018 & $0.09 \mu \mathrm{m}$ & $\mathrm{N} / \mathrm{A}$ & $2560 \times 1536$ & $\mathrm{~N} / \mathrm{A}$ & 60 & 67 & Moving Object Detection With Pre-defined Areas & ISS \\
\hline 26 & 2019 & $\mathrm{~N} / \mathrm{A}$ & $\begin{array}{l}\text { ZTE Nubia } \\
\text { UINX511 J }\end{array}$ & $5344 \times 3000$ & $\mathrm{~N} / \mathrm{A}$ & 120 & $\mathrm{~N} / \mathrm{A}$ & $\begin{array}{c}\text { Classroom Emotion with Cloud-Based Facial } \\
\text { Recognizer }\end{array}$ & ISS \\
\hline 27 & 2019 & $\mathrm{~N} / \mathrm{A}$ & $\begin{array}{c}\text { DJI PHANTOM } 3 \\
\text { PRO }\end{array}$ & $4000 \times 3000$ & $\mathrm{~N} / \mathrm{A}$ & $\mathrm{N} / \mathrm{A}$ & $\mathrm{N} / \mathrm{A}$ & vehicle Stacking Estimation & ISS \\
\hline 28 & 2020 & $\mathrm{~N} / \mathrm{A}$ & OV2710-1 E & $1920 \times 1080$ & 40 & 30 & 69 & Nuclear Radiation Detection & ISS \\
\hline 29 & 2020 & $\mathrm{~N} / \mathrm{A}$ & GS3-U3-23 S6 C-C & $1920 \times 1200$ & $\mathrm{~N} / \mathrm{A}$ & 162 & $\mathrm{~N} / \mathrm{A}$ & Contact less Neonatal Pulse Rate Sensing & ISS \\
\hline 30 & 2020 & $\mathrm{~N} / \mathrm{A}$ & OV9653 & $1300 \times 1028$ & 40 & 15 to 120 & 62 & HODET & ISS \\
\hline
\end{tabular}


Table 1. Cont.

\begin{tabular}{|c|c|c|c|c|c|c|c|c|c|}
\hline S. No. & Year & Technology & Camera Module & Resolution & SNR (dB) & $\begin{array}{l}\text { Frame Rate } \\
\text { (fps) }\end{array}$ & $\begin{array}{l}\text { Dynamic } \\
\text { Range (dB) }\end{array}$ & Application Name/Target & Field \\
\hline 31 & 2012 & $0.18 \mu \mathrm{m}$ & $\mathrm{N} / \mathrm{A}$ & $368 \times 368$ & $\mathrm{~N} / \mathrm{A}$ & 10 & 49.2 & Autonomous Micro Digital Sun Sensor & Space \\
\hline 32 & 2013 & $0.35 \mu \mathrm{m}$ & $\mathrm{N} / \mathrm{A}$ & $256 \times 256$ & $\mathrm{~N} / \mathrm{A}$ & $\mathrm{N} / \mathrm{A}$ & $\mathrm{N} / \mathrm{A}$ & Lightning Detection and Imaging & Space \\
\hline 33 & 2013 & $0.18 \mu \mathrm{m}$ & $\mathrm{N} / \mathrm{A}$ & $320 \times 128$ & $\mathrm{~N} / \mathrm{A}$ & $\mathrm{N} / \mathrm{A}$ & 126 & STAR Tracking & Space \\
\hline 34 & 2016 & $\mathrm{~N} / \mathrm{A}$ & CMV20000 & $5120 \times 3840$ & 41.8 & 0.45 & 66 & MARS 2020 Mission: EECAM & Space \\
\hline 35 & 2017 & $\mathrm{~N} / \mathrm{A}$ & $\begin{array}{l}\text { MT9 M001 C12 } \\
\text { STM }\end{array}$ & $1280 \times 1024$ & 45 & 30 & 68.2 & Cube SAT Remote Sensing Imagers & Space \\
\hline 36 & 2018 & $\mathrm{~N} / \mathrm{A}$ & CMV4000 & $2048 \times 2048$ & $\mathrm{~N} / \mathrm{A}$ & 180 & 60 & $\begin{array}{l}\text { Cloud Monitoring Camera (CMC) System for } \\
\text { Imaging Satellites }\end{array}$ & Space \\
\hline 37 & 2019 & $0.11 \mu \mathrm{m}$ & $\mathrm{N} / \mathrm{A}$ & $3000 \times 3000$ & 45 & $\mathrm{~N} / \mathrm{A}$ & 72.4 & Radiation Tolerant Sensor & Space \\
\hline 38 & 2019 & $\mathrm{~N} / \mathrm{A}$ & IMX 264 & $2464 \times 2056$ & $\mathrm{~N} / \mathrm{A}$ & 60 & $\mathrm{~N} / \mathrm{A}$ & Nanospacecraft Asteroid Flybys & Space \\
\hline 39 & 2019 & $\mathrm{~N} / \mathrm{A}$ & OV9630 & $1280 \times 1024$ & 54 & 15 & 60 & Mezn Sat for monitoring Green House Gases & Space \\
\hline 40 & 2020 & $\mathrm{~N} / \mathrm{A}$ & CIS2521 F & $2560 \times 2160$ & $\mathrm{~N} / \mathrm{A}$ & 100 & $>86$ & ASTERIA-A Space Telescope & Space \\
\hline 41 & 2010 & $\mathrm{~N} / \mathrm{A}$ & OV9653 & $1300 \times 1028$ & 40 & 30 & 62 & Wireless Aerial Image System & Millitary \\
\hline 42 & 2013 & $\mathrm{~N} / \mathrm{A}$ & OV7725 & $640 \times 480$ & 50 & 60 & 60 & IPASS & Millitary \\
\hline 43 & 2014 & $\mathrm{~N} / \mathrm{A}$ & $\mathrm{N} / \mathrm{A}$ & $640 \times 512$ & $\mathrm{~N} / \mathrm{A}$ & $\mathrm{N} / \mathrm{A}$ & $\mathrm{N} / \mathrm{A}$ & Banpil Camera & Millitary \\
\hline 44 & 2016 & $\mathrm{~N} / \mathrm{A}$ & MPT 50 & $640 \times 512$ & $\mathrm{~N} / \mathrm{A}$ & $\mathrm{N} / \mathrm{A}$ & $\mathrm{N} / \mathrm{A}$ & MWIR Detector for MissileApplications & Millitary \\
\hline 45 & 2016 & $\mathrm{~N} / \mathrm{A}$ & PHOTRON SA4 & $1024 \times 1024$ & 80 & 3600 & $\mathrm{~N} / \mathrm{A}$ & IN-SITU High velocity Rifle Bullets & Millitary \\
\hline 48 & 2018 & $\mathrm{~N} / \mathrm{A}$ & ESN-0510 & $640 \times 480$ & $\mathrm{~N} / \mathrm{A}$ & 30 & $\mathrm{~N} / \mathrm{A}$ & Sticky Bomb Detection & Millitary \\
\hline 49 & 2019 & $0.18 \mu \mathrm{m}$ & $\mathrm{N} / \mathrm{A}$ & $64 \times 64$ & $\mathrm{~N} / \mathrm{A}$ & $200 \mathrm{k}$ & $\mathrm{N} / \mathrm{A}$ & Gun Muzzle Flash Detection System & Millitary \\
\hline 50 & 2020 & $\mathrm{~N} / \mathrm{A}$ & MC1362 & $1280 \times 1024$ & $\mathrm{~N} / \mathrm{A}$ & $200 \mathrm{hz}$ & 90 & Critical Part Detection of Reconnaissance Balloon & Millitary \\
\hline 51 & 2010 & $\mathrm{~N} / \mathrm{A}$ & C328 R & $640 \times 480$ & $\mathrm{~N} / \mathrm{A}$ & $\mathrm{N} / \mathrm{A}$ & $\mathrm{N} / \mathrm{A}$ & Tigercense & WSN \\
\hline 52 & 2011 & $0.18 \mu \mathrm{m}$ & $\mathrm{N} / \mathrm{A}$ & $64 \times 64$ & $\mathrm{~N} / \mathrm{A}$ & 100 & $\mathrm{~N} / \mathrm{A}$ & On Chip Moving object Detection \& Localization & WSN \\
\hline 53 & 2011 & $\mathrm{~N} / \mathrm{A}$ & MT9 D131 & $1600 \times 1200$ & 42.3 & 15 & 71 & MasliNET & WSN \\
\hline 54 & 2012 & $0.6 \mu \mathrm{m}$ & $\mathrm{N} / \mathrm{A}$ & $384 \times 288$ & $\mathrm{~N} / \mathrm{A}$ & $\mathrm{N} / \mathrm{A}$ & $\mathrm{N} / \mathrm{A}$ & Eco-Hydrological Monitoring & WSN \\
\hline 55 & 2012 & $\mathrm{~N} / \mathrm{A}$ & C $328-7640$ & $640 \times 480$ & 46 & 30 & 62 & Monitoring Pest Insect Traps & WSN \\
\hline 56 & 2013 & $\mathrm{~N} / \mathrm{A}$ & MT9 M001 & $1280 \times 1024$ & $>45$ & 30 & $>62$ & River Surface Target Enhancement & WSN \\
\hline 57 & 2014 & $\mathrm{~N} / \mathrm{A}$ & OV5642 & $2592 \times 1944$ & 50 & 15 & 40 & TrustEYE.M4 & WSN \\
\hline 58 & 2014 & $\mathrm{~N} / \mathrm{A}$ & OV7725 & $640 \times 480$ & 50 & 60 & 60 & Wild life Inventory & WSN \\
\hline 59 & 2019 & $\mathrm{~N} / \mathrm{A}$ & IMX178 LLJ-C & $3088 \times 2064$ & $\mathrm{~N} / \mathrm{A}$ & 60 & $\mathrm{~N} / \mathrm{A}$ & Monitoring light pollution from small UAVs & WSN \\
\hline 60 & 2019 & $\mathrm{~N} / \mathrm{A}$ & $\begin{array}{c}\text { ELP-USBFHD04 } \\
\text { H-L170 }\end{array}$ & $1920 \times 1080$ & 39 & 30 & 72.4 & SoilCam & WSN \\
\hline
\end{tabular}


Table 2. Field wise mapping of CMOS image sensor model.

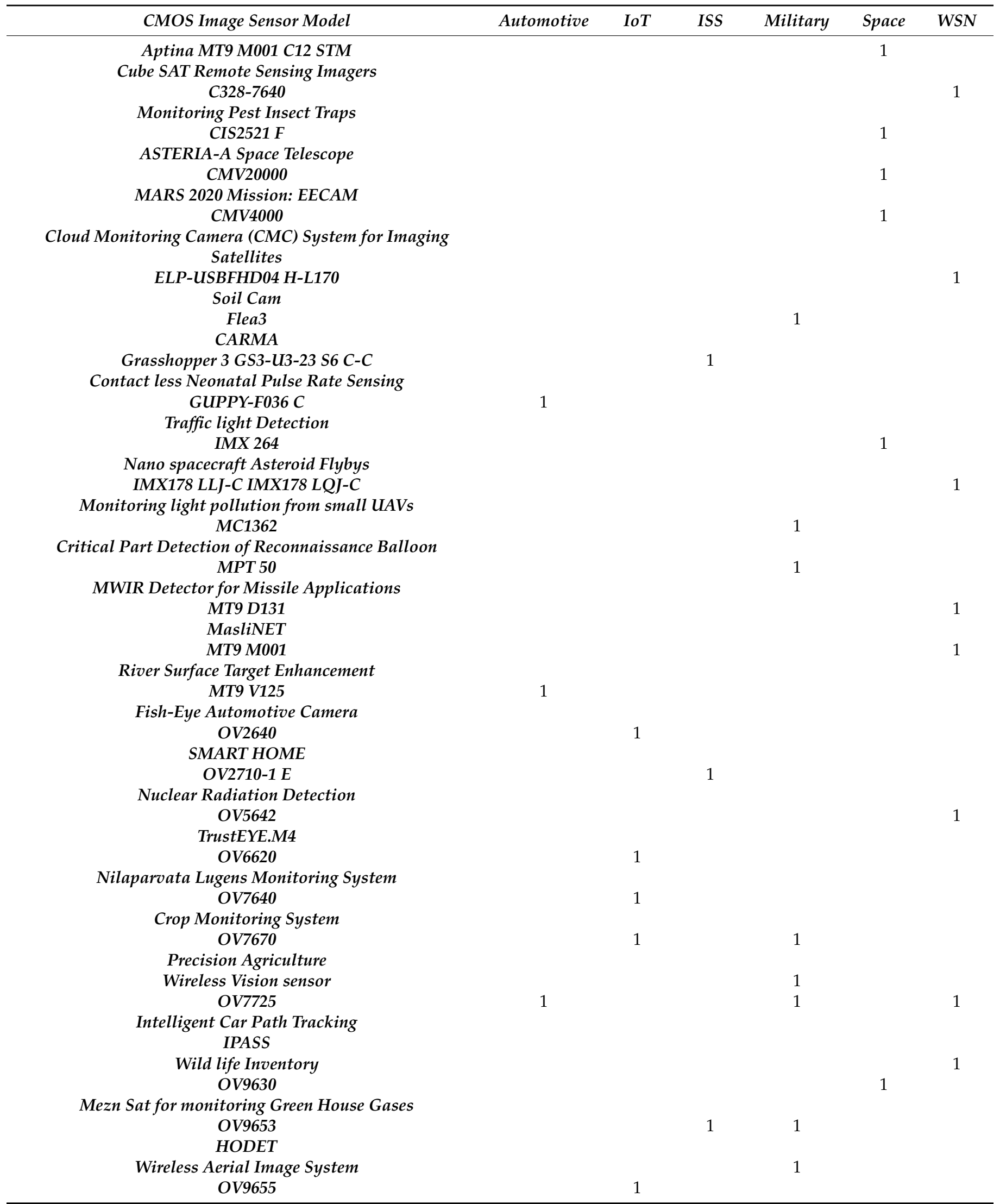


Table 2. Cont.

\begin{tabular}{|c|c|c|c|c|c|c|}
\hline CMOS Image Sensor Model & Automotive & IoT & ISS & Military & Space & WSN \\
\hline \multicolumn{7}{|l|}{ Smart Camera Networks (SCN) } \\
\hline PHOTRON SA4 & & & & 1 & & \\
\hline \multicolumn{7}{|l|}{ IN-SITU High velocity Rifle Bullets } \\
\hline Sekonix Camera SF3324-101 & & 1 & & & & \\
\hline \multicolumn{7}{|l|}{$C U b E$} \\
\hline ucam-II & & & 1 & & & \\
\hline Visual surveillance and intrusion detection & & & & & & \\
\hline Grand Total (33) & 3 & 6 & 4 & 7 & 6 & 7 \\
\hline
\end{tabular}

\section{Discussion}

\subsection{Camera Models}

From the data collected in our literature survey, we have mapped the CIS models incorporated in surveillance systems for various fields shown in Table 2. The remarkable models among them are EECAM for MARS 2020 mission, CUbE for driverless transportation of employers inside the industrial environment.

\subsection{Future Directions}

CIS has been used in many applications. It is technically improving very rapidly and being in more application areas. Quanta image sensors have evolved and have been developing gradually to overcome existing disadvantages compared to CCD. Due to the COVID outbreak, contactless technology is of interest in all applications, especially image capture, surveillance, money transactions like IRIS scanners and face recognition during the pandemic. New improvements were made in the medical field, and new algorithms are being implemented, such as face mask recognition in classrooms, especially for kids in school and playing zones.

\section{Conclusions}

Charge-coupled devices (CCD) played a vital role in many applications until CMOS image sensors came into existence. However, CIS still has some shortcomings to replacing CCDs in essential fields like the medical field and space, etc. To overcome CISs' shortcoming, s various technological advancements have been introduced during the last decade and have made CIS a leading good competitor to CCD in the present market. CIS is highly in demand in all reputed cameras and high spectral imaging applications due to its low manufacturing cost and size.

Author Contributions: This work has been primarily conducted by S.B.S. (Susrutha Babu Sukhavasi) and S.B.S. (Suparshya Babu Sukhavasi), under the supervision of K.E., S.B.S. (Susrutha Babu Sukhavasi) and S.B.S. (Suparshya Babu Sukhavasi) wrote the manuscript. Extensive discussions about algorithms and techniques presented in this paper took place among the authors S.B.S. (Susrutha Babu Sukhavasi), S.B.S. (Suparshya Babu Sukhavasi), K.E., S.A., and A.E. over the past year. All authors have read and agreed to the published version of the manuscript.

Funding: This research received no external funding.

Institutional Review Board Statement: Not Applicable.

Informed Consent Statement: Not Applicable.

Data Availability Statement: The data used in this review is from published primary studies, which are available in the public domain.

Acknowledgments: The authors acknowledge the University of Bridgeport for providing the necessary resources to carry this research conducted under the supervision of Khaled Elleithy.

Conflicts of Interest: The authors declare no conflict of interest. 


\author{
Abbreviations \\ The following abbreviations are used in this manuscript: \\ CIS CMOS Image Sensor \\ DR Dynamic Range \\ SNR Signal to Noise Ratio \\ FPS Frames Per Second \\ $\mathrm{dB} \quad$ Decibel \\ $\mathrm{mm} \quad$ Millimeter \\ $\mu \mathrm{m} \quad$ Micrometer \\ v/lux.sec Volts per luminance. second \\ CCD Charge Coupled Devices \\ CMOS Complementary Metal Oxide Semiconductor \\ IoT Internet of Things \\ ISS Intelligent Surveillance Systems \\ WSN Wireless Sensor Networks \\ BSI Back Side Illumination \\ WDR Wide Dynamic Range
}




\section{Appendix A}

Table A1. Taxonomy of the current literature.

\begin{tabular}{|c|c|c|c|c|c|c|c|c|c|c|}
\hline \multirow{2}{*}{ S. No. } & \multirow{2}{*}{ Year } & \multirow{2}{*}{ Camera Module } & \multicolumn{5}{|c|}{ Design Specifications } & \multirow{2}{*}{ Application Name/Target } & \multirow{2}{*}{ Field } & \multirow{2}{*}{ Reference } \\
\hline & & & Technology & Resolution & SNR & Frame Rate & Dynamic Range & & & \\
\hline 1 & 2020 & OV2710-1 E & - & YES & YES & YES & YES & Nuclear Radiation Detection & ISS & Zhangfa Yan et al. [25] \\
\hline 2 & 2020 & GS3-U3-23 S6 C-C & - & YES & No & YES & No & $\begin{array}{l}\text { Contact less Neonatal Pulse Rate } \\
\text { Sensing }\end{array}$ & ISS & M. Paul et al. [26] \\
\hline 3 & 2020 & OV9653 & - & YES & YES & YES & YES & HODET & ISS & Joseph st. Cyr et al. [1] \\
\hline 4 & 2020 & CIS2521 F & - & YES & No & YES & YES & ASTERIA-A Space Telescope & Space & Mary Knapp et al. [45] \\
\hline 5 & 2020 & MC1362 & - & YES & No & YES & YES & $\begin{array}{l}\text { Critical Part Detection of } \\
\text { Reconnaissance Balloon }\end{array}$ & Millitary & Hanyu Hong et al. [57] \\
\hline 6 & 2019 & OV2640 & - & YES & YES & YES & YES & SMART HOME & IoT & Vivek Raj et al. [34] \\
\hline 8 & 2019 & ZTE Nubia UINX511 J & - & YES & No & YES & No & $\begin{array}{l}\text { Classroom Emotion with Cloud-Based } \\
\text { Facial Recognizer }\end{array}$ & ISS & C. Boonroungrut et al. [23] \\
\hline 9 & 2019 & DJI PHANTOM 3 PRO & - & YES & No & No & No & Vehicle Stacking Estimation & ISS & Brain S. Freeman et al. [24] \\
\hline 10 & 2019 & - & $0.11 \mu \mathrm{m}$ & YES & YES & No & YES & Radiation Tolerant Sensor & Space & Woo-Tae Kim et al. [42] \\
\hline 11 & 2019 & $\operatorname{IMX} 264$ & - & YES & No & YES & & Nanospacecraft Asteroid Flybys & Space & Mihkel Pajusalu et al. [43] \\
\hline 12 & 2019 & OV9630 & - & YES & YES & YES & YES & $\begin{array}{c}\text { Mezn Sat for monitoring Green House } \\
\text { Gases }\end{array}$ & Space & Halim Jallad et al. [4] \\
\hline 13 & 2019 & - & $0.18 \mu \mathrm{m}$ & YES & No & YES & No & Gun Muzzle Flash Detection System & Millitary & Alex Katz et al. [56] \\
\hline 14 & 2019 & IMX178 LLJ,QJ-C & - & YES & No & YES & No & $\begin{array}{c}\text { Monitoring light pollution from small } \\
\text { UAVs }\end{array}$ & WSN & Fiorentin et al. [76] \\
\hline 15 & 2019 & $\begin{array}{l}\text { ELP-USBFHD04 } \\
\text { H-L170 }\end{array}$ & - & YES & YES & YES & YES & SoilCam & WSN & Gazi Rahman et al. [77] \\
\hline 16 & 2018 & - & $0.11 \mu \mathrm{m}$ & YES & No & YES & No & Non Contact Heart rate Detection & Automotive & Chen Cao et al. [60] \\
\hline 17 & 2018 & OV7725 & - & YES & YES & YES & YES & Intelligent Car Path Tracking & Automotive & Zixin Mu et al. [68] \\
\hline 18 & 2018 & OV7670 & - & YES & YES & YES & YES & Precision Agriculture System Design & IoT & Arun M.Patokar et al. [33] \\
\hline 19 & 2018 & - & $0.09 \mu \mathrm{m}$ & YES & No & YES & YES & $\begin{array}{l}\text { Moving Object Detection With } \\
\text { Pre-defined Areas }\end{array}$ & ISS & Oichi Kumagai et al. [22] \\
\hline 20 & 2018 & CMV4000 & - & YES & No & YES & YES & $\begin{array}{l}\text { Cloud Monitoring Camera (CMC) } \\
\text { System for Imaging Satellites }\end{array}$ & Space & Alpesh vala et al. [41] \\
\hline 21 & 2018 & ESN-0510 & - & YES & No & YES & No & Sticky Bomb Detection & Millitary & Raed Majeed et al. [55] \\
\hline 22 & 2017 & - & - & No & No & No & No & $\begin{array}{l}\text { Early Flood Detection \& Control } \\
\text { Monitoring }\end{array}$ & IoT & T M Thekkil et al. [32] \\
\hline 23 & 2017 & - & $0.18 \mu \mathrm{m}$ & YES & YES & YES & YES & Multi Resolution Mode & ISS & Daehyeok Kim et al. [21] \\
\hline 24 & 2017 & MT9 M001 C12 STM & - & YES & YES & YES & YES & Cube SAT Remote Sensing Imagers & Space & Dee W. Pack et al. [40] \\
\hline 25 & 2017 & Flea3 & - & YES & No & YES & YES & CARMA & Millitary & Shannon Johnson et al. [53] \\
\hline 26 & 2016 & - & $0.18 \mu \mathrm{m}$ & YES & No & No & YES & $\begin{array}{c}\text { Smart Image Sensor with Multi Point } \\
\text { Tracking (MPT) }\end{array}$ & IoT & Chin Yin et al. [31] \\
\hline 27 & 2016 & CMV20000 & - & YES & YES & YES & YES & MARS 2020 Mission: EECAM & Space & Mckinney et al. [39] \\
\hline
\end{tabular}


Table A1. Cont.

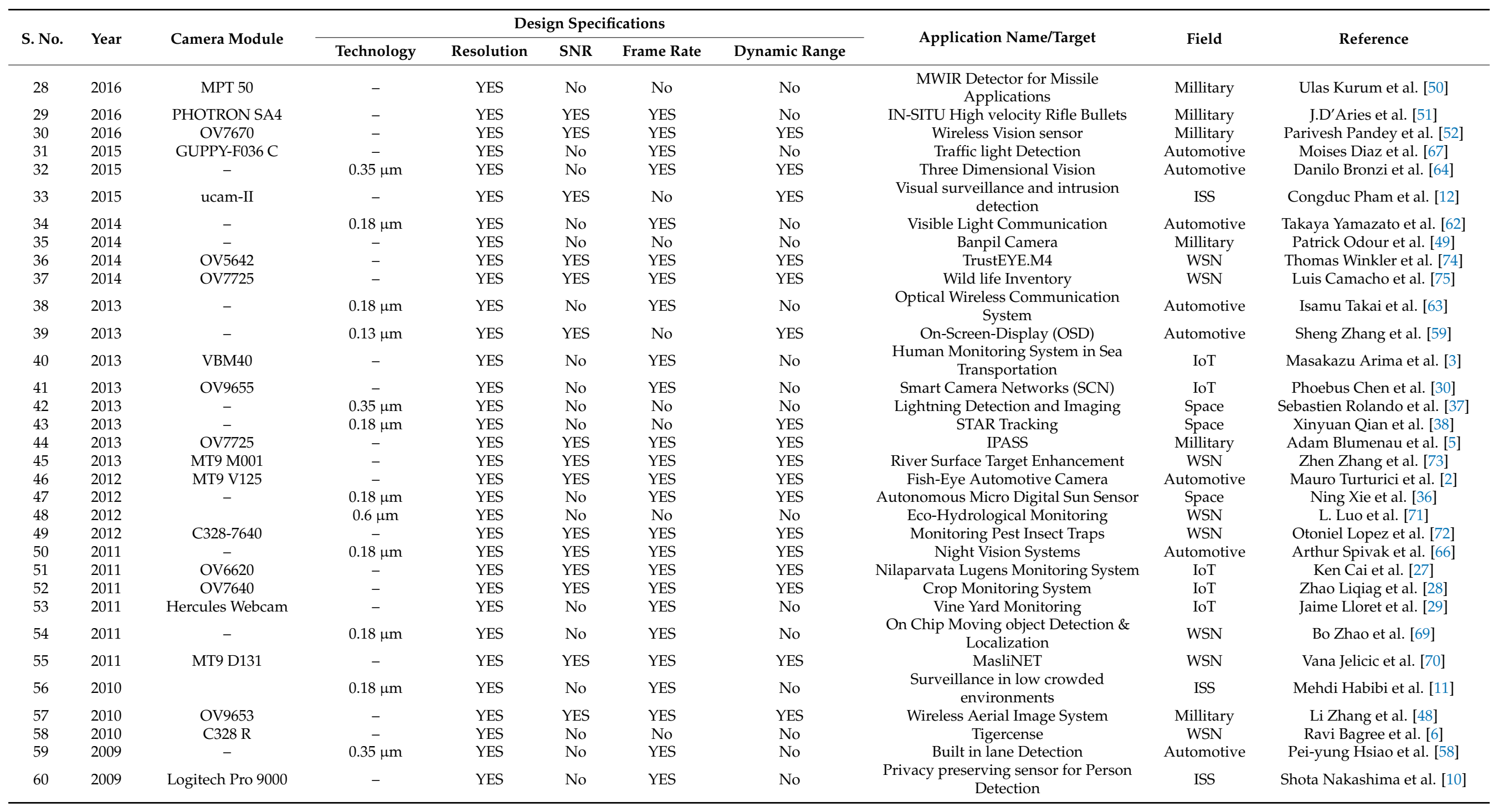


Table A2. Parametric data of CMOS image sensors used in surveillance system applications.

\begin{tabular}{|c|c|c|c|c|c|c|c|c|c|c|c|c|c|c|c|}
\hline Year & Technology & $\begin{array}{l}\text { Camera } \\
\text { Module }\end{array}$ & $\begin{array}{c}\text { Pixel Size } \\
(\mu \mathrm{m})\end{array}$ & Resolution & $\begin{array}{l}\text { Pixel } \\
\text { Pitch } \\
(\mu \mathrm{m})\end{array}$ & Area & $\begin{array}{c}\text { Power } \\
\text { (w/mw) }\end{array}$ & $\begin{array}{c}\text { Dark } \\
\text { Current } \\
\text { (mv/s) }\end{array}$ & $\begin{array}{l}\text { SNR } \\
(\mathrm{dB})\end{array}$ & $\begin{array}{c}\text { Conversion } \\
\text { Gain } \\
(\mu \mathrm{V} / \mathrm{e}-)\end{array}$ & $\begin{array}{c}\text { Sensitivity } \\
\text { (V/lux-s) }\end{array}$ & $\begin{array}{c}\text { Frame Rate } \\
\text { (fps) }\end{array}$ & $\begin{array}{c}\text { Dynamic } \\
\text { Range } \\
\text { (dB) }\end{array}$ & Field & $\begin{array}{c}\text { Application } \\
\text { Name/Target }\end{array}$ \\
\hline 2009 & N/A & $\begin{array}{l}\text { Logitech } \\
\text { Quickcam } \\
\text { Pro } 9000\end{array}$ & N/A & $1600 \times 1200$ & N/A & N/A & $\mathrm{N} / \mathrm{A}$ & N/A & $\mathrm{N} / \mathrm{A}$ & N/A & $\mathrm{N} / \mathrm{A}$ & $30 \mathrm{fps}$ & N/A & ISS & $\begin{array}{l}\text { Privacy } \\
\text { reserving } \\
\text { sensor for } \\
\text { Person } \\
\text { Detection }\end{array}$ \\
\hline 2010 & $0.18 \mu \mathrm{m}$ & N/A & $7.5 \times 7.5$ & $64 \times 64$ & 7.5 & $\underset{1 \mathrm{~mm} \times}{1 \mathrm{~mm}}$ & $0.5 \mathrm{mw}$ & N/A & N/A & N/A & $\mathrm{N} / \mathrm{A}$ & $30 \mathrm{fps}$ & $\mathrm{N} / \mathrm{A}$ & ISS & $\begin{array}{c}\text { Surveillance } \\
\text { in low } \\
\text { crowded } \\
\text { areas }\end{array}$ \\
\hline 2015 & N/A & ucam-II & $5.5 \times 5.5$ & $128 \times 128$ & 5.5 & $\begin{array}{c}27.5 \mathrm{~mm} \times \\
32.5 \mathrm{~mm}\end{array}$ & N/A & 25.2 & 44.2 & N/A & $\begin{array}{c}2.93 \mathrm{~V} / \\
\text { lux-s }\end{array}$ & N/A & 51 & ISS & $\begin{array}{c}\text { Visual } \\
\text { surveillance } \\
\text { and intrusion } \\
\text { detection }\end{array}$ \\
\hline 2017 & $0.18 \mu \mathrm{m}$ & N/A & $4.4 \times 4.4$ & $176 \times 144$ & 4.4 & $\begin{array}{c}2.35 \mathrm{~mm} \times \\
2.35 \mathrm{~mm}\end{array}$ & $\begin{array}{l}10 \mathrm{mw}- \\
\mathrm{HDR}\end{array}$ & N/A & $\begin{array}{l}47 \mathrm{~dB}- \\
\mathrm{HDR}\end{array}$ & N/A & - & $14 \mathrm{fps}$ & 61.8 & ISS & $\begin{array}{l}\text { Multi } \\
\text { Resolution } \\
\text { Mode }\end{array}$ \\
\hline 2018 & $0.09 \mu \mathrm{m}$ & N/A & $1.5 \times 1.5$ & $2560 \times 1536$ & 1.5 & $\begin{array}{c}4.48 \mathrm{~mm} \times \\
4.48 \mathrm{~mm}\end{array}$ & $95 \mathrm{mw}$ & N/A & $\mathrm{N} / \mathrm{A}$ & 55.8 & $\begin{array}{c}8033 \\
\text { e-/lux-s }\end{array}$ & $60 \mathrm{fps}$ & 67 & ISS & $\begin{array}{c}\text { Moving } \\
\text { Object } \\
\text { Detection } \\
\text { With } \\
\text { Pre-defined } \\
\text { Areas }\end{array}$ \\
\hline 2019 & $\mathrm{~N} / \mathrm{A}$ & $\begin{array}{l}\text { ZTE Nubia } \\
\text { UINX511 J }\end{array}$ & N/A & $5344 \times 3000$ & $\mathrm{~N} / \mathrm{A}$ & $\begin{array}{c}6.828 \mathrm{~mm} \times \\
6.828 \mathrm{~mm}\end{array}$ & $\mathrm{~N} / \mathrm{A}$ & N/A & $\mathrm{N} / \mathrm{A}$ & N/A & $\mathrm{N} / \mathrm{A}$ & $120 \mathrm{fps}$ & $\mathrm{N} / \mathrm{A}$ & ISS & $\begin{array}{c}\text { Classroom } \\
\text { Emotion with } \\
\text { Cloud-Based } \\
\text { Facial } \\
\text { Recognizer }\end{array}$ \\
\hline 2019 & N/A & $\begin{array}{c}\text { DJI } \\
\text { PHANTOM } 3 \\
\text { PRO }\end{array}$ & N/A & $4000 \times 3000$ & N/A & N/A & N/A & N/A & N/A & $\mathrm{N} / \mathrm{A}$ & N/A & $\mathrm{N} / \mathrm{A}$ & N/A & ISS & $\begin{array}{c}\text { vehicle } \\
\text { Stacking } \\
\text { Estimation }\end{array}$ \\
\hline 2020 & $\mathrm{~N} / \mathrm{A}$ & OV2710-1 E & $3 \times 3$ & $1920 \times 1080$ & 3 & $\begin{array}{c}5886 \mu \mathrm{m} \times \\
3276 \mu \mathrm{m}\end{array}$ & $350 \mathrm{~mW}$ & 20 & 40 & $\mathrm{~N} / \mathrm{A}$ & 3.7 & $30 \mathrm{fps}$ & 69 & ISS & $\begin{array}{c}\text { Nuclear } \\
\text { Radiation } \\
\text { Detection }\end{array}$ \\
\hline 2020 & N/A & $\begin{array}{c}\text { GS3-U3-23 S6 } \\
\text { C-C }\end{array}$ & $5.86 \times 5.86$ & $1920 \times 1200$ & 5.86 & N/A & N/A & N/A & N/A & N/A & N/A & $162 \mathrm{fps}$ & N/A & ISS & $\begin{array}{c}\text { Contact less } \\
\text { Neonatal } \\
\text { Pulse Rate } \\
\text { Sensing }\end{array}$ \\
\hline 2020 & N/A & OV9653 & $3.18 \times 3.18$ & $1300 \times 1028$ & 3.18 & $\begin{array}{c}4.13 \mathrm{~mm} \times \\
3.28 \mathrm{~mm}\end{array}$ & $50 \mathrm{mw}$ & 30 & 40 & N/A & 0.9 & $15-120 \mathrm{fps}$ & 62 & ISS & HODET \\
\hline
\end{tabular}


Table A2. Cont.

\begin{tabular}{|c|c|c|c|c|c|c|c|c|c|c|c|c|c|c|c|}
\hline Year & Technology & $\begin{array}{l}\text { Camera } \\
\text { Module }\end{array}$ & $\begin{array}{c}\text { Pixel Size } \\
\quad(\mu \mathrm{m})\end{array}$ & Resolution & $\begin{array}{l}\text { Pixel } \\
\text { Pitch } \\
(\mu \mathrm{m})\end{array}$ & Area & $\begin{array}{l}\text { Power } \\
(w / m w)\end{array}$ & $\begin{array}{c}\text { Dark } \\
\text { Current } \\
\text { (mv/s) }\end{array}$ & $\begin{array}{l}\text { SNR } \\
\text { (dB) }\end{array}$ & $\begin{array}{c}\text { Conversion } \\
\text { Gain } \\
(\mu \mathrm{V} / \mathrm{e}-)\end{array}$ & $\begin{array}{l}\text { Sensitivity } \\
\text { (V/lux-s) }\end{array}$ & $\begin{array}{c}\text { Frame Rate } \\
\text { (fps) }\end{array}$ & $\begin{array}{c}\text { Dynamic } \\
\text { Range } \\
\text { (dB) }\end{array}$ & Field & $\begin{array}{l}\text { Application } \\
\text { Name/Target }\end{array}$ \\
\hline 2011 & N/A & OV6620 & $9.0 \times 8.2$ & $356 \times 292$ & 9.0 & $\begin{array}{c}3.1 \mathrm{~mm} \times \\
2.5 \mathrm{~mm}\end{array}$ & $<80 \mathrm{mw}$ & $\begin{array}{l}<0.2 \mathrm{nA} \\
/ \mathrm{cm}^{2}\end{array}$ & $>48$ & N/A & N/A & $60 \mathrm{fps}$ & $>72$ & IoT & $\begin{array}{l}\text { Nilaparvata } \\
\text { Lugens } \\
\text { Monitoring } \\
\text { System }\end{array}$ \\
\hline 2011 & N/A & OV7640 & $5.6 \times 5.6$ & $640 \times 480$ & 5.6 & $\begin{array}{c}3.6 \mathrm{~mm} \times \\
2.7 \mathrm{~mm}\end{array}$ & $40 \mathrm{mw}$ & 30 & 46 & N/A & $\begin{array}{c}\text { 3.0-Black } \\
\text { /Color-1.12 }\end{array}$ & $30 \mathrm{fps}$ & 62 & IoT & $\begin{array}{l}\text { Crop } \\
\text { Monitoring } \\
\text { System }\end{array}$ \\
\hline 2011 & N/A & $\begin{array}{l}\text { Hercules } \\
\text { Webcam }\end{array}$ & N/A & $1280 \times 960$ & N/A & N/A & N/A & N/A & N/A & N/A & N/A & $30 \mathrm{fps}$ & N/A & IoT & $\begin{array}{l}\text { Vine Yard } \\
\text { Monitoring }\end{array}$ \\
\hline 2013 & N/A & VBM40 & N/A & $1280 \times 960$ & $\mathrm{~N} / \mathrm{A}$ & $\begin{array}{c}132 \mathrm{~mm} \times \\
152 \mathrm{~mm}\end{array}$ & N/A & N/A & N/A & $\mathrm{N} / \mathrm{A}$ & $0.6 \mathrm{~V} / \mathrm{lux}$ & $30 \mathrm{fps}$ & N/A & IoT & $\begin{array}{l}\text { Human } \\
\text { Monitoring } \\
\text { System in Sea } \\
\text { Transporta- } \\
\text { tion }\end{array}$ \\
\hline 2013 & N/A & OV9655 & $3.18 \times 3.18$ & $1280 \times 1024$ & 3.18 & $\begin{array}{c}5145 \mu \mathrm{m} \times \\
6145 \mu \mathrm{m}\end{array}$ & $90 \mathrm{mw}$ & N/A & N/A & N/A & N/A & $15 \mathrm{fps}$ & N/A & IoT & $\begin{array}{c}\text { Smart } \\
\text { Camera } \\
\text { Networks } \\
(\mathrm{SCN})\end{array}$ \\
\hline 2016 & $0.18 \mu \mathrm{m}$ & N/A & $20 \times 20$ & $64 \times 64$ & 20 & N/A & N/A & N/A & N/A & N/A & N/A & N/A & 96.7 & IoT & $\begin{array}{l}\text { Smart Image } \\
\text { Sensor with } \\
\text { Multi Point } \\
\text { Tracking } \\
\text { (MPT) }\end{array}$ \\
\hline 2017 & N/A & N/A & N/A & N/A & N/A & N/A & N/A & N/A & $\mathrm{N} / \mathrm{A}$ & $\mathrm{N} / \mathrm{A}$ & N/A & N/A & $\mathrm{N} / \mathrm{A}$ & IoT & $\begin{array}{l}\text { Early Flood } \\
\text { Detection \& } \\
\text { Control } \\
\text { Monitoring }\end{array}$ \\
\hline 2018 & N/A & OV7670 & $3.6 \times 3.6$ & $640 \times 480$ & 3.6 & $\begin{array}{c}2.36 \mathrm{~mm} \times \\
1.76 \mathrm{~mm}\end{array}$ & N/A & $12 \mathrm{mv} / \mathrm{s}$ & 40 & N/A & $\begin{array}{c}1.1 \\
\text { V/lux-sec }\end{array}$ & $30 \mathrm{fps}$ & 52 & IoT & $\begin{array}{l}\text { Precision } \\
\text { Agriculture } \\
\text { System } \\
\text { Design }\end{array}$ \\
\hline 2019 & $\mathrm{~N} / \mathrm{A}$ & OV2640 & $2.2 \times 2.2$ & $1600 \times 1200$ & 2.2 & $\begin{array}{c}3590 \mu \mathrm{m} \times \\
2684 \mu \mathrm{m}\end{array}$ & $125 \mathrm{~mW}$ & 15 & 40 & N/A & 0.6 & $15 \mathrm{fps}$ & 50 & IoT & $\begin{array}{l}\text { SMART } \\
\text { HOME }\end{array}$ \\
\hline 2019 & N/A & $\begin{array}{c}\text { Sekonix } \\
\text { SF3324-101 } \\
\end{array}$ & $3 \times 3$ & $1928 \times 1208$ & 3 & $\begin{array}{c}26 \mathrm{~mm} \times \\
26 \mathrm{~mm}\end{array}$ & N/A & N/A & N/A & N/A & N/A & N/A & $\mathrm{N} / \mathrm{A}$ & IoT & CUbE \\
\hline 2012 & $0.18 \mu \mathrm{m}$ & N/A & N/A & $368 \times 368$ & N/A & $\begin{array}{c}5 \mathrm{~mm} \times \\
5 \mathrm{~mm}\end{array}$ & $\begin{array}{c}21.34 \mathrm{mw} \\
\text { @ Acqui- } \\
\text { sition } \\
21.39 \mathrm{~mW} \\
\text { @ } \\
\text { Tracking }\end{array}$ & N/A & N/A & N/A & N/A & $10 \mathrm{fps}$ & $49.2 \mathrm{~dB}$ & Space & $\begin{array}{c}\text { Autonomous } \\
\text { Micro Digital } \\
\text { Sun Sensor }\end{array}$ \\
\hline
\end{tabular}


Table A2. Cont.

\begin{tabular}{|c|c|c|c|c|c|c|c|c|c|c|c|c|c|c|c|}
\hline Year & Technology & $\begin{array}{l}\text { Camera } \\
\text { Module }\end{array}$ & $\begin{array}{c}\text { Pixel Size } \\
(\mu \mathrm{m})\end{array}$ & Resolution & $\begin{array}{l}\text { Pixel } \\
\text { Pitch } \\
(\mu \mathrm{m})\end{array}$ & Area & $\begin{array}{c}\text { Power } \\
\text { (w/mw) }\end{array}$ & $\begin{array}{c}\text { Dark } \\
\text { Current } \\
\text { (mv/s) }\end{array}$ & $\begin{array}{l}\text { SNR } \\
\text { (dB) }\end{array}$ & $\begin{array}{c}\text { Conversion } \\
\text { Gain } \\
(\mu \mathrm{V} / \mathrm{e}-)\end{array}$ & $\begin{array}{c}\text { Sensitivity } \\
\text { (V/lux-s) }\end{array}$ & $\begin{array}{c}\text { Frame Rate } \\
\text { (fps) }\end{array}$ & $\begin{array}{c}\text { Dynamic } \\
\text { Range } \\
\text { (dB) }\end{array}$ & Field & $\begin{array}{c}\text { Application } \\
\text { Name/Target }\end{array}$ \\
\hline 2013 & $0.35 \mu \mathrm{m}$ & N/A & $60 \times 60$ & $256 \times 256$ & 60 & $\begin{array}{c}17.8 \mathrm{~mm} \times \\
17.8 \mathrm{~mm}\end{array}$ & N/A & N/A & N/A & 5.7 & N/A & N/A & $\mathrm{N} / \mathrm{A}$ & Space & $\begin{array}{l}\text { Lightning } \\
\text { Detection and } \\
\text { Imaging }\end{array}$ \\
\hline 2013 & $0.18 \mu \mathrm{m}$ & $\mathrm{N} / \mathrm{A}$ & $5 \times 5$ & $320 \times 128$ & 5 & $\begin{array}{c}2.5 \mathrm{~mm} \times \\
2.5 \mathrm{~mm}\end{array}$ & $247 \mathrm{~mW}$ & $1537 \mathrm{fA}$ & N/A & N/A & 0.25 & $\mathrm{~N} / \mathrm{A}$ & $126 \mathrm{~dB}$ & Space & $\begin{array}{c}\text { STAR } \\
\text { Tracking }\end{array}$ \\
\hline 2016 & N/A & CMV20000 & $6.4 \times 6.4$ & $5120 \times 3840$ & 6.4 & $\begin{array}{c}32.77 \mathrm{~mm} \times \\
24.58 \mathrm{~mm}\end{array}$ & $<3 w$ & $125 \mathrm{e}-/ \mathrm{s}$ & $41.8 \mathrm{~dB}$ & 0.25 & $\mathrm{~N} / \mathrm{A}$ & $0.45 \mathrm{fps}$ & $66 \mathrm{~dB}$ & Space & $\begin{array}{c}\text { MARS } 2020 \\
\text { Mission: } \\
\text { EECAM }\end{array}$ \\
\hline 2018 & N/A & CMV4000 & $5.5 \times 5.5$ & $2048 \times 2048$ & 5.5 & N/A & $650 \mathrm{mw}$ & $125 \mathrm{e}-/ \mathrm{s}$ & N/A & 0.075 LSB/e- & 5.56 & $180 \mathrm{fps}$ & $60 \mathrm{~dB}$ & Space & $\begin{array}{l}\text { Cloud } \\
\text { Monitoring } \\
\text { Camera } \\
\text { (CMC) } \\
\text { System for } \\
\text { Imaging } \\
\text { Satellites }\end{array}$ \\
\hline 2019 & $0.11 \mu \mathrm{m}$ & N/A & $6.5 \times 6.5$ & $3000 \times 3000$ & 6.5 & $\begin{array}{c}22 \mathrm{~mm} \times \\
22 \mathrm{~mm}\end{array}$ & $\mathrm{~N} / \mathrm{A}$ & $\mathrm{N} / \mathrm{A}$ & $45 \mathrm{~dB}$ & 8.55 & $\mathrm{~N} / \mathrm{A}$ & $\mathrm{N} / \mathrm{A}$ & $72.4 \mathrm{~dB}$ & Space & $\begin{array}{c}\text { Radiation } \\
\text { Tolerant } \\
\text { Sensor }\end{array}$ \\
\hline 2019 & N/A & IMX 264 & $3.45 \times 3.45$ & $2464 \times 2056$ & 3.45 & N/A & N/A & $\mathrm{N} / \mathrm{A}$ & N/A & N/A & 0.915 & $60 \mathrm{fps}$ & N/A & Space & $\begin{array}{l}\text { Nano } \\
\text { spacecraft } \\
\text { Asteroid } \\
\text { Flybys }\end{array}$ \\
\hline 2019 & N/A & OV9630 & $4.2 \times 4.2$ & $1280 \times 1024$ & 4.2 & $\begin{array}{c}5.4 \mathrm{~mm} \times \\
4.3 \mathrm{~mm}\end{array}$ & $150 \mathrm{~mW}$ & $28 \mathrm{mv}$ & $54 \mathrm{~dB}$ & $\mathrm{~N} / \mathrm{A}$ & 1 & $15 \mathrm{fps}$ & $60 \mathrm{~dB}$ & Space & $\begin{array}{c}\text { Mezn Sat for } \\
\text { monitoring } \\
\text { Green House } \\
\text { Gases }\end{array}$ \\
\hline 2020 & $\mathrm{~N} / \mathrm{A}$ & CIS2521 F & $6.5 \times 6.5$ & $2560 \times 2160$ & 6.5 & $\mathrm{~N} / \mathrm{A}$ & $\mathrm{N} / \mathrm{A}$ & $35 \mathrm{e}-/ \mathrm{s}$ & $\mathrm{N} / \mathrm{A}$ & $\mathrm{N} / \mathrm{A}$ & $\mathrm{N} / \mathrm{A}$ & $100 \mathrm{fps}$ & $>86 \mathrm{~dB}$ & Space & $\begin{array}{l}\text { ASTERIA-A } \\
\text { Space } \\
\text { Telescope }\end{array}$ \\
\hline 2010 & N/A & OV9653 & $3.18 \times 3.18$ & $1300 \times 1028$ & 3.18 & $\begin{array}{l}4.13 \mathrm{~mm} \times \\
3.28 \mathrm{~mm}\end{array}$ & $50 \mathrm{mw}$ & 30 & $40 \mathrm{~dB}$ & $\mathrm{~N} / \mathrm{A}$ & 0.9 & $30 \mathrm{fps}$ & $62 \mathrm{~dB}$ & Military & $\begin{array}{c}\text { Wireless } \\
\text { Aerial Image } \\
\text { System }\end{array}$ \\
\hline 2013 & $\mathrm{~N} / \mathrm{A}$ & OV7725 & $6.0 \times 6.0$ & $640 \times 480$ & 6 & $\begin{array}{c}3984 \mu \mathrm{m} \times \\
2952 \mu \mathrm{m}\end{array}$ & $120 \mathrm{MW}$ & $40 \mathrm{mV} / \mathrm{s}$ & $50 \mathrm{~dB}$ & $\mathrm{~N} / \mathrm{A}$ & 3 & $60 \mathrm{fps}$ & $60 \mathrm{~dB}$ & Military & IPASS \\
\hline 2014 & $\mathrm{~N} / \mathrm{A}$ & $\mathrm{N} / \mathrm{A}$ & $\mathrm{N} / \mathrm{A}$ & $640 \times 512$ & N/A & $\begin{array}{c}9.6 \mathrm{~mm} \times \\
7.7 \mathrm{~mm}\end{array}$ & $\mathrm{~N} / \mathrm{A}$ & N/A & N/A & $\mathrm{N} / \mathrm{A}$ & N/A & $\mathrm{N} / \mathrm{A}$ & $\mathrm{N} / \mathrm{A}$ & Military & $\begin{array}{c}\text { Banpil } \\
\text { Camera }\end{array}$ \\
\hline
\end{tabular}


Table A2. Cont.

\begin{tabular}{|c|c|c|c|c|c|c|c|c|c|c|c|c|c|c|c|}
\hline Year & Technology & $\begin{array}{l}\text { Camera } \\
\text { Module }\end{array}$ & $\begin{array}{c}\text { Pixel Size } \\
(\mu \mathrm{m})\end{array}$ & Resolution & $\begin{array}{l}\text { Pixel } \\
\text { Pitch } \\
(\mu \mathrm{m})\end{array}$ & Area & $\begin{array}{c}\text { Power } \\
\text { (w/mw) }\end{array}$ & $\begin{array}{c}\text { Dark } \\
\text { Current } \\
\text { (mv/s) }\end{array}$ & $\begin{array}{l}\mathrm{SNR} \\
(\mathrm{dB})\end{array}$ & $\begin{array}{c}\text { Conversion } \\
\text { Gain } \\
(\mu \mathrm{V} / \mathrm{e}-)\end{array}$ & $\begin{array}{c}\text { Sensitivity } \\
\text { (V/lux-s) }\end{array}$ & $\begin{array}{c}\text { Frame Rate } \\
\text { (fps) }\end{array}$ & $\begin{array}{c}\text { Dynamic } \\
\text { Range } \\
\text { (dB) }\end{array}$ & Field & $\begin{array}{c}\text { Application } \\
\text { Name/Target }\end{array}$ \\
\hline 2016 & N/A & MPT 50 & $15 \times 15$ & $640 \times 512$ & 15 & N/A & N/A & N/A & N/A & $\mathrm{N} / \mathrm{A}$ & $\mathrm{N} / \mathrm{A}$ & N/A & N/A & Military & $\begin{array}{c}\text { MWIR } \\
\text { Detector for } \\
\text { Missile } \\
\text { Applications }\end{array}$ \\
\hline 2016 & $\mathrm{~N} / \mathrm{A}$ & $\begin{array}{c}\text { PHOTRON } \\
\text { SA4 }\end{array}$ & $20 \times 20$ & $1024 \times 1024$ & 20 & $\begin{array}{c}160 \mathrm{~mm} \times \\
153 \mathrm{~mm}\end{array}$ & N/A & N/A & $80 \mathrm{~dB}$ & N/A & N/A & $3600 \mathrm{fps}$ & $\mathrm{N} / \mathrm{A}$ & Military & $\begin{array}{c}\text { IN-SITU } \\
\text { High velocity } \\
\text { Rifle Bullets }\end{array}$ \\
\hline 2016 & N/A & OV7670 & $3.6 \times 3.6$ & $640 \times 480$ & 3.6 & $\begin{array}{c}2.36 \mathrm{~mm} \times \\
1.76 \mathrm{~mm}\end{array}$ & $60 \mathrm{~mW}$ & 12 & $46 \mathrm{~dB}$ & $\mathrm{~N} / \mathrm{A}$ & 1.3 & $15 \mathrm{fps}$ & $52 \mathrm{~dB}$ & Military & $\begin{array}{c}\text { Wireless } \\
\text { Vision sensor }\end{array}$ \\
\hline 2017 & $\mathrm{~N} / \mathrm{A}$ & Flea3 & $1.55 \times 1.55$ & $4000 \times 3000$ & 1.55 & $\begin{array}{c}29 \mathrm{~mm} \times \\
29 \mathrm{~mm}\end{array}$ & $\mathrm{~N} / \mathrm{A}$ & $\mathrm{N} / \mathrm{A}$ & N/A & N/A & N/A & $15 \mathrm{fps}$ & $66.46 \mathrm{~dB}$ & Military & CARMA \\
\hline 2018 & N/A & ESN-0510 & $\mathrm{N} / \mathrm{A}$ & $640 \times 480$ & $\mathrm{~N} / \mathrm{A}$ & N/A & N/A & $\mathrm{N} / \mathrm{A}$ & N/A & N/A & N/A & $30 \mathrm{fps}$ & N/A & Military & $\begin{array}{l}\text { Sticky Bomb } \\
\text { Detection }\end{array}$ \\
\hline 2019 & $0.18 \mu \mathrm{m}$ & N/A & $54 \times 54$ & $64 \times 64$ & 54 & $\begin{array}{l}5 \mathrm{~mm} \times \\
5.5 \mathrm{~mm}\end{array}$ & $\mathrm{~N} / \mathrm{A}$ & N/A & $\mathrm{N} / \mathrm{A}$ & N/A & N/A & $200 \mathrm{kfps}$ & N/A & Military & $\begin{array}{c}\text { Gun Muzzle } \\
\text { Flash } \\
\text { Detection } \\
\text { System }\end{array}$ \\
\hline 2020 & $\mathrm{~N} / \mathrm{A}$ & MC1362 & $14 \times 14$ & $1280 \times 1024$ & 14 & $\begin{array}{c}17.92 \mathrm{~mm} \times \\
14.34 \mathrm{~mm}\end{array}$ & N/A & 0.2 & N/A & N/A & 25 & $200 \mathrm{hz}$ & $90 \mathrm{~dB}$ & Military & $\begin{array}{c}\text { Critical Part } \\
\text { Detection of } \\
\text { Reconnais- } \\
\text { sance } \\
\text { Balloon }\end{array}$ \\
\hline 2009 & $0.35 \mu \mathrm{m}$ & N/A & $\begin{array}{r}13.1 \times \\
22.05\end{array}$ & $64 \times 64$ & $\mathrm{~N} / \mathrm{A}$ & $\begin{array}{c}2194.4 \mu \mathrm{m} \times \\
2389.8 \mu \mathrm{m}\end{array}$ & $\begin{array}{c}159.4 \\
\mathrm{mw}\end{array}$ & N/A & N/A & N/A & $\mathrm{N} / \mathrm{A}$ & $10 \mathrm{fps}$ & $\mathrm{N} / \mathrm{A}$ & Automotive & $\begin{array}{l}\text { Built in lane } \\
\text { Detection }\end{array}$ \\
\hline 2011 & $0.18 \mu \mathrm{m}$ & N/A & $\begin{array}{c}13.75 \times \\
13.75 \\
\end{array}$ & $128 \times 256$ & 8.5 & N/A & $10 \mathrm{mw}$ & $0.1 \mathrm{fA}$ & $51 \mathrm{db}$ & 72 & $\mathrm{~N} / \mathrm{A}$ & $60 \mathrm{fps}$ & $98 \mathrm{~dB}$ & Automotive & $\begin{array}{c}\text { Night Vision } \\
\text { Systems }\end{array}$ \\
\hline 2012 & $\mathrm{~N} / \mathrm{A}$ & MT9 V125 & $5.6 \times 5.6$ & $720 \times 480$ & 5.6 & $\begin{array}{c}3.63 \mathrm{~mm} \times \\
2.78 \mathrm{~mm}\end{array}$ & $320 \mathrm{mw}$ & N/A & $39 \mathrm{~dB}$ & $\mathrm{~N} / \mathrm{A}$ & $\mathrm{N} / \mathrm{A}$ & $30 \mathrm{fps}$ & $70 \mathrm{~dB}$ & Automotive & $\begin{array}{c}\text { Fish-Eye } \\
\text { Automotive } \\
\text { Camera }\end{array}$ \\
\hline 2013 & $0.18 \mu \mathrm{m}$ & N/A & $7.5 \times 7.5$ & $642 \times 480$ & 7.5 & $\begin{array}{c}7.5 \mathrm{~mm} \times \\
8.0 \mathrm{~mm}\end{array}$ & N/A & N/A & N/A & N/A & $\mathrm{N} / \mathrm{A}$ & $30 \mathrm{fps}$ & $\mathrm{N} / \mathrm{A}$ & Automotive & $\begin{array}{c}\text { Optical } \\
\text { Wireless Com } \\
\text { munication } \\
\text { System }\end{array}$ \\
\hline 2013 & $0.13 \mu \mathrm{m}$ & $\mathrm{N} / \mathrm{A}$ & $6.0 \times 6.0$ & $768 \times 576$ & 6 & $\mathrm{~N} / \mathrm{A}$ & $\mathrm{N} / \mathrm{A}$ & N/A & $45 \mathrm{db}$ & 95 & $\begin{array}{c}4.8 \\
\text { V/lux-sec }\end{array}$ & $\mathrm{N} / \mathrm{A}$ & $70 \mathrm{~dB}$ & Automotive & $\begin{array}{l}\text { On-Screen- } \\
\text { Display } \\
\text { (OSD) }\end{array}$ \\
\hline 2014 & $0.18 \mu \mathrm{m}$ & $\mathrm{N} / \mathrm{A}$ & $7.5 \times 7.5$ & $642 \times 480$ & 7.5 & $\begin{array}{c}7.5 \mathrm{~mm} \times \\
8.0 \mathrm{~mm}\end{array}$ & $\mathrm{~N} / \mathrm{A}$ & $\mathrm{N} / \mathrm{A}$ & $\mathrm{N} / \mathrm{A}$ & $\mathrm{N} / \mathrm{A}$ & $\mathrm{N} / \mathrm{A}$ & $60 \mathrm{fps}$ & $\mathrm{N} / \mathrm{A}$ & Automotive & $\begin{array}{c}\text { Visible Light } \\
\text { Communica- } \\
\text { tion }\end{array}$ \\
\hline 2015 & $\mathrm{~N} / \mathrm{A}$ & $\begin{array}{c}\text { GUPPY-F036 } \\
\text { C }\end{array}$ & $6.0 \times 6.0$ & $752 \times 480$ & 6 & N/A & N/A & N/A & N/A & N/A & N/A & $64 \mathrm{fps}$ & N/A & Automotive & $\begin{array}{l}\text { Traffic light } \\
\text { Detection }\end{array}$ \\
\hline
\end{tabular}


Table A2. Cont.

\begin{tabular}{|c|c|c|c|c|c|c|c|c|c|c|c|c|c|c|c|}
\hline Year & Technology & $\begin{array}{l}\text { Camera } \\
\text { Module }\end{array}$ & $\begin{array}{c}\text { Pixel Size } \\
(\mu \mathrm{m})\end{array}$ & Resolution & $\begin{array}{l}\text { Pixel } \\
\text { Pitch } \\
(\mu \mathrm{m})\end{array}$ & Area & $\begin{array}{c}\text { Power } \\
(\mathrm{w} / \mathrm{mw})\end{array}$ & $\begin{array}{c}\text { Dark } \\
\text { Current } \\
(\mathrm{mv} / \mathrm{s})\end{array}$ & $\begin{array}{l}\text { SNR } \\
(\mathrm{dB})\end{array}$ & $\begin{array}{c}\text { Conversion } \\
\text { Gain } \\
(\mu \mathrm{V} / \mathrm{e}-)\end{array}$ & $\begin{array}{l}\text { Sensitivity } \\
\text { (V/lux-s) }\end{array}$ & $\begin{array}{c}\text { Frame Rate } \\
\text { (fps) }\end{array}$ & $\begin{array}{c}\text { Dynamic } \\
\text { Range } \\
\text { (dB) }\end{array}$ & Field & $\begin{array}{c}\text { Application } \\
\text { Name/Target }\end{array}$ \\
\hline 2015 & $0.35 \mu \mathrm{m}$ & N/A & $150 \times 150$ & $64 \times 32$ & 150 & N/A & $4 \mathrm{w}$ & $\mathrm{N} / \mathrm{A}$ & $\mathrm{N} / \mathrm{A}$ & N/A & $\mathrm{N} / \mathrm{A}$ & $100 \mathrm{fps}$ & $110 \mathrm{~dB}$ & Automotive & $\begin{array}{c}\text { Three- } \\
\text { Dimensional } \\
\text { Vision }\end{array}$ \\
\hline 2018 & $0.11 \mu \mathrm{m}$ & N/A & $7.1 \times 7.1$ & $1280 \times 1024$ & 7.1 & $\begin{array}{c}12.6 \mathrm{~mm} \times \\
14.8 \mathrm{~mm}\end{array}$ & N/A & $\mathrm{N} / \mathrm{A}$ & $\mathrm{N} / \mathrm{A}$ & 99.2 & $\begin{array}{c}134.8 \\
\text { ke-/lux-s }\end{array}$ & $30 \mathrm{fps}$ & N/A & Automotive & $\begin{array}{c}\text { Non-Contact } \\
\text { Heart Rate } \\
\text { Detection }\end{array}$ \\
\hline 2018 & N/A & OV7725 & $6.0 \times 6.0$ & $640 \times 480$ & 6 & $\begin{array}{c}3984 \mu \mathrm{m} \times \\
2952 \mu \mathrm{m}\end{array}$ & $120 \mathrm{~mW}$ & $40 \mathrm{mv} / \mathrm{s}$ & $50 \mathrm{~dB}$ & N/A & $\begin{array}{c}3.8 \\
\text { V/lux-sec }\end{array}$ & $60 \mathrm{fps}$ & $60 \mathrm{~dB}$ & Automotive & $\begin{array}{l}\text { Intelligent } \\
\text { Car Path } \\
\text { Tracking }\end{array}$ \\
\hline 2010 & N/A & C $328 \mathrm{R}$ & N/A & $640 \times 480$ & 2 & $\begin{array}{c}20 \mathrm{~mm} \times \\
28 \mathrm{~mm}\end{array}$ & $\mathrm{~N} / \mathrm{A}$ & N/A & N/A & N/A & $\mathrm{N} / \mathrm{A}$ & $\mathrm{N} / \mathrm{A}$ & N/A & WSN & Tigercense \\
\hline 2011 & $0.18 \mu \mathrm{m}$ & N/A & $14 \times 14$ & $64 \times 64$ & 14 & $\begin{array}{c}1.5 \mathrm{~mm} \times \\
1.5 \mathrm{~mm}\end{array}$ & $0.4 \mathrm{mw}$ & $6.7 \mathrm{fA}$ & $\mathrm{N} / \mathrm{A}$ & N/A & $\begin{array}{c}0.11 \\
\text { V/lux-s }\end{array}$ & $100 \mathrm{fps}$ & N/A & WSN & $\begin{array}{c}\text { On Chip } \\
\text { Moving } \\
\text { object } \\
\text { Detection \& } \\
\text { localization }\end{array}$ \\
\hline 2011 & $\mathrm{~N} / \mathrm{A}$ & MT9 D131 & $2.8 \times 2.8$ & $1600 \times 1200$ & 2.8 & $\begin{array}{c}4.73 \mathrm{~mm} \times \\
3.52 \mathrm{~mm}\end{array}$ & $348 \mathrm{mw}$ & $\mathrm{N} / \mathrm{A}$ & $42.3 \mathrm{~dB}$ & $\mathrm{~N} / \mathrm{A}$ & $\begin{array}{c}1.0 \\
\text { V/lux-sec }\end{array}$ & $15 \mathrm{fps}$ & $71 \mathrm{~dB}$ & WSN & MasliNET \\
\hline 2012 & $0.6 \mu \mathrm{m}$ & $\mathrm{N} / \mathrm{A}$ & $24 \times 24$ & $384 \times 288$ & 24 & $\begin{array}{c}11.5 \mathrm{~mm} \times \\
7.7 \mathrm{~mm}\end{array}$ & $150 \mathrm{~mW}$ & N/A & $\mathrm{N} / \mathrm{A}$ & $\mathrm{N} / \mathrm{A}$ & $\mathrm{N} / \mathrm{A}$ & $\mathrm{N} / \mathrm{A}$ & $\mathrm{N} / \mathrm{A}$ & WSN & $\begin{array}{c}\text { Eco- } \\
\text { Hydrological } \\
\text { Monitoring }\end{array}$ \\
\hline 2012 & N/A & C328-7640 & $5.6 \times 5.6$ & $640 \times 480$ & 5.6 & $\begin{array}{c}3.6 \mathrm{~mm} \times \\
2.7 \mathrm{~mm}\end{array}$ & $40 \mathrm{mw}$ & $30 \mathrm{mv} / \mathrm{s}$ & $46 \mathrm{~dB}$ & $\mathrm{~N} / \mathrm{A}$ & $\begin{array}{c}3.0 \mathrm{~V} / \text { lux-s } \\
-\mathrm{B} \& \mathrm{~W}, 1.12 \\
\mathrm{~V} / \text { Lux-S } \\
\text {-Colour }\end{array}$ & $30 \mathrm{fps}$ & $62 \mathrm{~dB}$ & WSN & $\begin{array}{c}\text { Monitoring } \\
\text { Pest Insect } \\
\text { Traps }\end{array}$ \\
\hline 2013 & $\mathrm{~N} / \mathrm{A}$ & MT9 M001 & $5.2 \times 5.2$ & $1280 \times 1024$ & 5.2 & $\mathrm{~N} / \mathrm{A}$ & $325 \mathrm{mw}$ & 20-30 e-s & $>45 \mathrm{~dB}$ & $\mathrm{~N} / \mathrm{A}$ & $\begin{array}{c}1.8 \\
\text { v/lux-sec }\end{array}$ & $30 \mathrm{fps}$ & $>62 \mathrm{~dB}$ & WSN & $\begin{array}{l}\text { River Surface } \\
\text { Target } \\
\text { Enhancement }\end{array}$ \\
\hline 2014 & $\mathrm{~N} / \mathrm{A}$ & OV5642 & $1.4 \times 1.4$ & $2592 \times 1944$ & 1.4 & $\begin{array}{c}3673.6 \mu \mathrm{m} \times \\
2738.4 \mu \mathrm{m}\end{array}$ & N/A & N/A & $50 \mathrm{~dB}$ & N/A & $\begin{array}{c}0.6 \\
\text { V/lux-sec }\end{array}$ & $15 \mathrm{fps}$ & $40 \mathrm{~dB}$ & WSN & TrustEYE.M4 \\
\hline 2014 & N/A & OV7725 & $6 \times 6$ & $640 \times 480$ & 6 & $\begin{array}{c}3984 \mu \mathrm{m} \times \\
2952 \mu \mathrm{m}\end{array}$ & $120 \mathrm{~mW}$ & $40 \mathrm{mv} / \mathrm{s}$ & $50 \mathrm{~dB}$ & $\mathrm{~N} / \mathrm{A}$ & $\begin{array}{c}3.8 \\
\text { V/lux-sec } \\
\end{array}$ & $60 \mathrm{fps}$ & $60 \mathrm{~dB}$ & WSN & $\begin{array}{l}\text { Wild life } \\
\text { Inventory }\end{array}$ \\
\hline 2019 & N/A & $\begin{array}{c}\text { IMX178 } \\
\text { LLJ-C } \\
\text { IMX178 } \\
\text { LQJ-C }\end{array}$ & $2.4 \times 2.4$ & $3088 \times 2064$ & 2.4 & $\begin{array}{c}8.92 \mathrm{~mm} \times \\
8.92 \mathrm{~mm}\end{array}$ & $\mathrm{~N} / \mathrm{A}$ & $\mathrm{N} / \mathrm{A}$ & $\mathrm{N} / \mathrm{A}$ & $\mathrm{N} / \mathrm{A}$ & $0.38,0.425$ & $60 \mathrm{fps}$ & $\mathrm{N} / \mathrm{A}$ & WSN & $\begin{array}{l}\text { Monitoring } \\
\text { light } \\
\text { pollution } \\
\text { from small } \\
\text { UAVs }\end{array}$ \\
\hline 2019 & N/A & $\begin{array}{c}\text { ELP- } \\
\text { USBFHD04 } \\
\text { H-L170 }\end{array}$ & $2.2 \times 2.2$ & $1920 \times 1080$ & 2.2 & $\begin{array}{c}32 \mathrm{~mm} \times \\
32 \mathrm{~mm}\end{array}$ & N/A & N/A & $39 \mathrm{~dB}$ & $\mathrm{~N} / \mathrm{A}$ & $\begin{array}{c}1.9 \\
\text { v/lux-sec }\end{array}$ & $30 \mathrm{fps}$ & $72.4 \mathrm{~dB}$ & WSN & SoilCam \\
\hline
\end{tabular}




\section{References}

1. Cyr, J.S.; Vanderpool, J.; Chen, Y.; Li, X. HODET: Hybrid object detection and tracking using mmWave radar and visual sensors. In Sensors and Systems for Space Applications XIII; International Society for Optics and Photonics: Bellingham, WA, USA, 2020; p. 114220I.

2. Turturici, M.; Saponara, S.; Fanucci, L.; Franchi, E. Low-power embedded system for real-time correction of fish-eye automotive cameras. In Proceedings of the 2012 Design, Automation \& Test in Europe Conference \& Exhibition (DATE), Dresden, Germany, 12-16 March 2012; pp. 340-341.

3. Arima, M.; Kii, S. Development of an Autonomous Human Monitoring System for Preventative Safety in Sea Transportation. In Proceedings of the International Conference on Offshore Mechanics and Arctic Engineering, Nantes, France, 9-14 June 2013; p. V02AT02A040.

4. Jallad, A.-H.; Marpu, P.; Abdul Aziz, Z.; Al Marar, A.; Awad, M. MeznSat-A 3U CubeSat for Monitoring Greenhouse Gases Using Short Wave Infra-Red Spectrometry: Mission Concept and Analysis. Aerospace 2019, 6, 118. [CrossRef]

5. Blumenau, A.; Ishak, A.; Limone, B.; Mintz, Z.; Russell, C.; Sudol, A.; Linton, R.; Lai, L.; Padir, T.; Van Hook, R. Design and implementation of an intelligent portable aerial surveillance system (ipass). In Proceedings of the 2013 IEEE Conference on Technologies for Practical Robot Applications (TePRA), Woburn, MA, USA, 22-23 April 2013; pp. 1-6.

6. $\quad$ Bagree, R.; Jain, V.R.; Kumar, A.; Ranjan, P. Tigercense: Wireless image sensor network to monitor tiger movement. In Proceedings of the International Workshop on Real-world Wireless Sensor Networks, Colombo, Sri Lanka, 16-17 December 2010; pp. 13-24.

7. Ohta, J. Smart CMOS Image Sensors and Applications; CRC Press: Boca Raton, FL, USA, 2020.

8. Bigas, M.; Cabruja, E.; Forest, J.; Salvi, J. Review of CMOS image sensors. Microelectron. J. 2006, 37, 433-451. [CrossRef]

9. El Gamal, A.; Eltoukhy, H. CMOS image sensors. IEEE Circuits Devices Mag. 2005, 21, 6-20. [CrossRef]

10. Nakashima, S.; Kitazono, Y.; Zhang, L.; Serikawa, S. Development of privacy-preserving sensor for person detection. Procedia-Soc. Behav. Sci. 2010, 2, 213-217.

11. Habibi, M. A low power smart CMOS image sensor for surveillance applications. In Proceedings of the 20106 th Iranian Conference on Machine Vision and Image Processing, Isfahan, Iran, 27-28 October 2010; pp. 1-4.

12. Pham, C. Low cost wireless image sensor networks for visual surveillance and intrusion detection applications. In Proceedings of the 2015 IEEE 12th International Conference on Networking, Sensing and Control, Taipei, Taiwan, 9-11 April 2015 ; pp. 376-381.

13. Rahimi, M.; Baer, R.; Iroezi, O.I.; Garcia, J.C.; Warrior, J.; Estrin, D.; Srivastava, M. Cyclops: In situ image sensing and interpretation in wireless sensor networks. In Proceedings of the 3rd International Conference on Embedded Networked Sensor Systems, SenSys05, San Diego, CA, USA, 2-5 November 2005; pp. 192-204.

14. Chen, P.; Ahammad, P.; Boyer, C.; Huang, S.-I.; Lin, L.; Lobaton, E.; Meingast, M.; Oh, S.; Wang, S.; Yan, P. CITRIC: A lowbandwidth wireless camera network platform. In Proceedings of the 2008 Second ACM/IEEE International Conference on Distributed Smart Cameras, Stanford, CA, USA, 7-11 September 2008; pp. 1-10.

15. Evidence Embedding Technology, Seed-Eye Board, a Multimedia Wsn Device. Available online: http://rtn.sssup.it/index.php/ hardware/seed-eye (accessed on 20 December 2013).

16. Feng, W.-C.; Kaiser, E.; Feng, W.C.; Baillif, M.L. Panoptes: Scalable low-power video sensor networking technologies. ACM Trans. Multimed. Comput. Commun. Appl. (TOMM) 2005, 1, 151-167. [CrossRef]

17. Paniga, S.; Borsani, L.; Redondi, A.; Tagliasacchi, M.; Cesana, M. Experimental evaluation of a video streaming system for wireless multimedia sensor networks. In Proceedings of the 2011 The 10th IFIP Annual Mediterranean Ad Hoc Networking Workshop, Sicily, Italy, 12-15 June 2011; pp. 165-170.

18. Rowe, A.; Goel, D.; Rajkumar, R. Firefly mosaic: A vision-enabled wireless sensor networking system. In Proceedings of the 28th IEEE International Real-Time Systems Symposium (RTSS 2007), Tucson, AZ, USA, 3-6 December 2007; pp. $459-468$.

19. Rodríguez-Vázquez, Á.; Domínguez-Castro, R.; Jiménez-Garrido, F.; Morillas, S.; Listán, J.; Alba, L.; Utrera, C.; Espejo, S.; Romay, R. The Eye-RIS CMOS vision system. In Analog Circuit Design; Springer: Berlin/Heidelberg, Germany, $2008 ;$ pp. 15-32.

20. Kleihorst, R.; Abbo, A.; Schueler, B.; Danilin, A. Camera mote with a high-performance parallel processor for real-time framebased video processing. In Proceedings of the 2007 First ACM/IEEE International Conference on Distributed Smart Cameras, Vienna, Austria, 25-28 September 2007; pp. 109-116.

21. Kim, D.; Song, M.; Choe, B.; Kim, S.Y. A multi-resolution mode CMOS image sensor with a novel two-step single-slope ADC for intelligent surveillance systems. Sensors 2017, 17, 1497. [CrossRef]

22. Kumagai, O.; Niwa, A.; Hanzawa, K.; Kato, H.; Futami, S.; Ohyama, T.; Imoto, T.; Nakamizo, M.; Murakami, H.; Nishino, T. A 1/4-inch 3.9 Mpixel low-power event-driven back-illuminated stacked CMOS image sensor. In Proceedings of the 2018 IEEE International Solid-State Circuits Conference-(ISSCC), San Francisco, CA, USA, 11-15 February 2018; pp. 86-88.

23. Boonroungrut, C.; Oo, T.T. Exploring Classroom Emotion with Cloud-Based Facial Recognizer in the Chinese Beginning Class: A Preliminary Study. Int. J. Instr. 2019, 12, 947-958.

24. Freeman, B.S.; Al Matawah, J.A.; Al Najjar, M.; Gharabaghi, B.; Thé, J. Vehicle stacking estimation at signalized intersections with unmanned aerial systems. Int. J. Transp. Sci. Technol. 2019, 8, 231-249. [CrossRef]

25. Yan, Z.; Wei, Q.; Huang, G.; Hu, Y.; Zhang, Z.; Dai, T. Nuclear radiation detection based on uncovered CMOS camera under dynamic scene. Nucl. Instrum. Methods Phys. Res. Sect. A Accel. Spectrometers Detect. Assoc. Equip. 2020, 956, 163383. [CrossRef] 
26. Paul, M.; Karthik, S.; Joseph, J.; Sivaprakasam, M.; Kumutha, J.; Leonhardt, S.; Antink, C.H. Non-contact sensing of neonatal pulse rate using camera-based imaging: A clinical feasibility study. Physiol. Meas. 2020, 41, 024001.

27. Cai, K.; Wu, X.; Liang, X.; Wang, K. Hardware Design of Sensor Nodes in the Nilaparvata Lugens Monitoring System Based on the Internet of Things. In Advanced Electrical and Electronics Engineering; Springer: Berlin/Heidelberg, Germany, 2011; pp. 571-578.

28. Liqiang, Z.; Shouyi, Y.; Leibo, L.; Zhen, Z.; Shaojun, W. A crop monitoring system based on wireless sensor network. Procedia Environ. Sci. 2011, 11, 558-565. [CrossRef]

29. Lloret, J.; Bosch, I.; Sendra, S.; Serrano, A. A wireless sensor network for vineyard monitoring that uses image processing. Sensors 2011, 11, 6165-6196. [PubMed]

30. Chen, P.; Hong, K.; Naikal, N.; Sastry, S.S.; Tygar, D.; Yan, P.; Yang, A.Y.; Chang, L.-C.; Lin, L.; Wang, S. A low-bandwidth camera sensor platform with applications in smart camera networks. ACM Trans. Sens. Netw. 2013, 9, 1-23. [CrossRef]

31. Yin, C.; Chiu, C.-F.; Hsieh, C.-C. A 0.5 V, 14.28-kframes/s, 96.7-dB smart image sensor with array-level image signal processing for IoT applications. IEEE Trans. Electron Devices 2016, 63, 1134-1140.

32. Thekkil, T.M.; Prabakaran, N. Real-time WSN based early flood detection and control monitoring system. In Proceedings of the 2017 International Conference on Intelligent Computing, Instrumentation and Control Technologies (ICICICT), Kannur, India, 6-7 July 2017; pp. 1709-1713.

33. Patokar, A.M.; Gohokar, V.V. Precision agriculture system design using wireless sensor network. In Information and Communication Technology; Springer: Berlin/Heidelberg, Germany, 2018; pp. 169-177.

34. Raj, V.; Chandran, A.; RS, A. IoT Based Smart Home Using Multiple Language Voice Commands. In Proceedings of the 2019 2nd International Conference on Intelligent Computing, Instrumentation and Control Technologies (ICICICT), Kerala, India, 5-6 July 2019; pp. 1595-1599.

35. Hartmannsgruber, A.; Seitz, J.; Schreier, M.; Strauss, M.; Balbierer, N.; Hohm, A. CUbE: A Research Platform for Shared Mobility and Autonomous Driving in Urban Environments. In Proceedings of the 2019 IEEE Intelligent Vehicles Symposium (IV), Paris, France, 9-12 June 2019; pp. 2315-2322.

36. Xie, N.; Theuwissen, A.J. An autonomous microdigital sun sensor by a cmos imager in space application. IEEE Trans. Electron Devices 2012, 59, 3405-3410.

37. Rolando, S.; Goiffon, V.; Magnan, P.; Corbière, F.; Molina, R.; Tulet, M.; Bréart-de-Boisanger, M.; Saint-Pé, O.; Guiry, S.; Larnaudie, F. Smart CMOS image sensor for lightning detection and imaging. Appl. Opt. 2013, 52, C16-C23. [CrossRef]

38. Qian, X.; Yu, H.; Chen, S.; Low, K.S. An adaptive integration time CMOS image sensor with multiple readout channels. IEEE Sens. J. 2013, 13, 4931-4939.

39. Maki, J.; McKinney, C.; Sellar, R.; Copley-Woods, D.; Gruel, D.; Nuding, D. Enhanced Engineering Cameras (EECAMs) for the Mars 2020 Rover. In Proceedings of the 3rd International Workshop on Instrumentation for Planetary Mission, Pasadena, CA, USA, 24-27 October 2016; Volume 1980, p. 4132. Available online: http:/ / adsabs.harvard.edu/abs/2016LPICo1980M (accessed on 20 September 2020).

40. Pack, D.; Ardila, D.; Herman, E.; Rowen, D.; Welle, R.; Wiktorowicz, S.; Hattersley, B. Two Aerospace Corporation CubeSat remote Sensing Imagers: CUMULOS and R3. Available online: https://digitalcommons.usu.edu/smallsat/2017/all2017/82/ (accessed on 20 September 2020).

41. Vala, A.; Patel, A.; Gosai, R.; Chaudharia, J.; Mewada, H.; Mahant, K. A low-cost and efficient cloud monitoring camera system design for imaging satellites. Int. J. Remote Sens. 2019, 40, 2739-2758.

42. Kim, W.-T.; Park, C.; Lee, H.; Lee, I.; Lee, B.-G. A high full well capacity CMOS image sensor for space applications. Sensors 2019, 19, 1505.

43. Pajusalu, M.; Slavinskis, A. Characterization of Asteroids Using Nanospacecraft Flybys and Simultaneous Localization and Mapping. In Proceedings of the 2019 IEEE Aerospace Conference, Big Sky, MT, USA, 2-9 March 2019; pp. 1-9.

44. The CubeSat Program. CubeSat Design Specification Rev. 13. California Polytechnic State University. 2014. Available online: http:/ / www.cubesat.org/s/cds_rev13_final2.pdf (accessed on 16 May 2019).

45. Knapp, M.; Seager, S.; Demory, B.-O.; Krishnamurthy, A.; Smith, M.W.; Pong, C.M.; Bailey, V.P.; Donner, A.; Di Pasquale, P.; Campuzano, B. Demonstrating high-precision photometry with a CubeSat: ASTERIA observations of 55 Cancrie. Astron. J. 2020, 160, 23.

46. Ricker, G.; Winn, J. Transiting Exoplanet Survey Satellite. J. Astron. Telesc. Instrum. Syst. 2014, 1, 014003. [CrossRef]

47. Catala, C.; Appourchaux, T.; Consortium, P.M. PLATO: PLAnetary Transits and Oscillations of stars. In Proceedings of the Journal of Physics, Conference Series, Aix-en-Provence, France, 27 June-2 July 2010; p. 012084.

48. Zhang, L.; Liu, C.; Qian, G. The portable wireless aerial image transmission system based on DSP. In Proceedings of the 2010 International Conference on Microwave and Millimeter Wave Technology, Chengdu, China, 8-11 May 2010; pp. 1591-1594.

49. Oduor, P.; Mizuno, G.; Olah, R.; Dutta, A.K. Development of low-cost high-performance multispectral camera system at Banpil. In Proceedings of the Image Sensing Technologies, Materials, Devices, Systems, and Applications, Baltimore, MD, USA, 11 June 2014; p. 910006.

50. Kürüm, U. Scenario-based analysis of binning in MWIR detectors for missile applications. In Proceedings of the Infrared Imaging Systems, Design, Analysis, Modeling, and Testing XXVII, Baltimore, MD, USA, 3 May 2016; p. 982000. 
51. Lawrence, J.; Miller, S.R.; Robertson, R.; Singh, B.; Nagarkar, V.V. High frame-rate real-time x-ray imaging of in situ high-velocity rifle bullets. In Proceedings of the Anomaly Detection and Imaging with X-Rays (ADIX), Baltimore, MD, USA, 12 May 2016 ; p. $98470 G$.

52. Pandey, P.; Laxmi, V. Design of low cost and power efficient Wireless vision Sensor for surveillance and monitoring. In Proceedings of the 2016 International Conference on Computation of Power, Energy Information and Communication (ICCPEIC), Chennai, India, 20-21 April 2016; pp. 113-117.

53. Johnson, S.; Stroup, R.; Gainer, J.J.; De Vries, L.D.; Kutzer, M.D. Design of a Robotic Catch and Release Manipulation Architecture (CARMA). In Proceedings of the ASME International Mechanical Engineering Congress and Exposition, Tampa, FL, USA, 3-9 November 2017; p. V04BT05A010.

54. Valenti, M.; Bethke, B.; Dale, D.; Frank, A.; McGrew, J.; Ahrens, S.; How, J.P.; Vian, J. The MIT indoor multi-vehicle flight testbed. In Proceedings of the 2007 IEEE International Conference on Robotics and Automation, Roma, Italy, 10-14 April 2007; pp. 2758-2759.

55. Majeed, R.; Hatem, H.; Mohammed, M. Automatic Detection System to the Sticky Bomb. Comput. Sci. Eng. 2018, 8, 17-22.

56. Katz, A.; Shoham, A.; Vainstein, C.; Birk, Y.; Leitner, T.; Fenigstein, A.; Nemirovsky, Y. Passive CMOS Single Photon Avalanche Diode Imager for a Gun Muzzle Flash Detection System. IEEE Sens. J. 2019, 19, 5851-5858. [CrossRef]

57. Hong, H.; Shi, J.; Liu, Z.; Zhang, Y.; Wu, J. A real-time critical part detection for the blurred image of infrared reconnaissance balloon with boundary curvature feature analysis. J. Real-Time Image Process. 2020, 1-16. [CrossRef]

58. Hsiao, P.-Y.; Cheng, H.-C.; Huang, S.-S.; Fu, L.-C. CMOS image sensor with a built-in lane detector. Sensors 2009, 9, $1722-1737$. [PubMed]

59. Zhang, S.; Zhang, H.; Chen, B.; Shao, D.; Xu, C. On-Screen-display (OSD) and SPI interface on CMOS image sensor for automobile application. In Proceedings of the 2013 Fifth International Conference on Computational Intelligence, Communication Systems and Networks, Madrid, Spain, 5-7 June 2013; pp. 405-408.

60. Cao, C.; Shirakawa, Y.; Tan, L.; Seo, M.-W.; Kagawa, K.; Yasutomi, K.; Kosugi, T.; Aoyama, S.; Teranishi, N.; Tsumura, N. A two-tap NIR lock-in pixel CMOS image sensor with background light cancelling capability for non-contact heart rate detection. In Proceedings of the 2018 IEEE Symposium on VLSI Circuits, Honolulu, HI, USA, 18-22 June 2018; pp. 75-76.

61. Friel, M.; Hughes, C.; Denny, P.; Jones, E.; Glavin, M. Automatic calibration of fish-eye cameras from automotive video sequences. IET Intell. Transp. Syst. 2010, 4, 136-148. [CrossRef]

62. Yamazato, T.; Takai, I.; Okada, H.; Fujii, T.; Yendo, T.; Arai, S.; Andoh, M.; Harada, T.; Yasutomi, K.; Kagawa, K. Image-sensorbased visible light communication for automotive applications. IEEE Commun. Mag. 2014, 52, 88-97.

63. Takai, I.; Ito, S.; Yasutomi, K.; Kagawa, K.; Andoh, M.; Kawahito, S. LED and CMOS image sensor based optical wireless communication system for automotive applications. IEEE Photonics J. 2013, 5, 6801418. [CrossRef]

64. Bronzi, D.; Zou, Y.; Villa, F.; Tisa, S.; Tosi, A.; Zappa, F. Automotive three-dimensional vision through a single-photon counting SPAD camera. IEEE Trans. Intell. Transp. Syst. 2015, 17, 782-795.

65. Kwon, D.; Park, S.; Baek, S.; Malaiya, R.K.; Yoon, G.; Ryu, J.-T. A study on development of the blind spot detection system for the IoT-based smart connected car. In Proceedings of the 2018 IEEE International Conference on Consumer Electronics (ICCE), Las Vegas, NV, USA, 12-14 June 2018; pp. 1-4.

66. Spivak, A.; Belenky, A.; Fish, A.; Yadid-Pecht, O. A wide-dynamic-range CMOS image sensor with gating for night vision systems. IEEE Trans. Circuits Syst. II Express Briefs 2011, 58, 85-89.

67. Diaz-Cabrera, M.; Cerri, P.; Medici, P. Robust real-time traffic light detection and distance estimation using a single camera. Expert Syst. Appl. 2015, 42, 3911-3923. [CrossRef]

68. $\mathrm{Mu}, \mathrm{Z}$; $\mathrm{Li}$, Z. Intelligent tracking car path planning based on Hough transform and improved PID algorithm. In Proceedings of the 2018 5th International Conference on Systems and Informatics (ICSAI), Nanjing, China, 10-12 November 2018; pp. 24-28.

69. Zhao, B.; Zhang, X.; Chen, S.; Low, K.-S.; Zhuang, H. A 64\$, \times $\backslash, \$ 64$ CMOS Image Sensor With On-Chip Moving Object Detection and Localization. IEEE Trans. Circuits Syst. Video Technol. 2011, 22, 581-588. [CrossRef]

70. Jeličić, V.; Ražov, T.; Oletić, D.; Kuri, M.; Bilas, V. MasliNET: A Wireless Sensor Network based environmental monitoring system. In Proceedings of the 2011 34th International Convention MIPRO, Opatija, Croatia, 23-27 May 2011; pp. 150-155.

71. Luo, L.; Zhang, Y.; Zhu, W. E-Science application of wireless sensor networks in eco-hydrological monitoring in the Heihe River basin, China. IET Sci. Meas. Technol. 2012, 6, 432-439. [CrossRef]

72. López, O.; Rach, M.M.; Migallon, H.; Malumbres, M.P.; Bonastre, A.; Serrano, J.J. Monitoring pest insect traps by means of low-power image sensor technologies. Sensors 2012, 12, 15801-15819. [CrossRef]

73. Zhang, Z.; Wang, X.; Fan, T.; Xu, L. River surface target enhancement and background suppression for unseeded LSPIV. Flow Meas. Instrum. 2013, 30, 99-111. [CrossRef]

74. Winkler, T.; Erdélyi, A.; Rinner, B. TrustEYE. M4: Protecting the sensor-Not the camera. In Proceedings of the 2014 11th IEEE International Conference on Advanced Video and Signal Based Surveillance (AVSS), Seoul, Korea, 26-29 August 2014; pp. 159-164.

75. Camacho, L.; Baquerizo, R.; Palomino, J.; Zarzosa, M. Deployment of a set of camera trap networks for wildlife inventory in western amazon rainforest. IEEE Sens. J. 2017, 17, 8000-8007. [CrossRef] 
76. Fiorentin, P.; Bettanini, C.; Bogoni, D.; Aboudan, A.; Colombatti, G. Calibration of an imaging system for monitoring light pollution from small UAVs. In Proceedings of the 2019 IEEE 5th International Workshop on Metrology for AeroSpace (MetroAeroSpace), Torino, Italy, 19-21 June 2019; pp. 267-271.

77. Rahman, G.; Sohag, H.; Chowdhury, R.; Wahid, K.A.; Dinh, A.; Arcand, M.; Vail, S. SoilCam: A Fully Automated Minirhizotron using Multispectral Imaging for Root Activity Monitoring. Sensors 2020, 20, 787. [CrossRef]

78. Semiconductor and Computer Engineering. Available online: https://en.wikichip.org/wiki/technology_node (accessed on 20 September 2020).

79. Image Resolution. Available online: https:/ / en.wikipedia.org/wiki/Image_resolution (accessed on 20 September 2020).

80. Dupuis, Y.; Savatier, X.; Vasseur, P. Feature subset selection applied to model-free gait recognition. Image Vis. Comput. 2013, 31, 580-591. [CrossRef]

81. Rida, I.; Jiang, X.; Marcialis, G.L. Human body part selection by group lasso of motion for model-free gait recognition. IEEE Signal Process. Lett. 2015, 23, 154-158. [CrossRef]

82. Rida, I.; Almaadeed, N.; Almaadeed, S. Robust gait recognition: A comprehensive survey. IET Biom. 2018, 8, 14-28. [CrossRef]

83. Wan, C.; Wang, L.; Phoha, V.V. A survey on gait recognition. ACM Comput. Surv. (CSUR) 2018, 51, 1-35. [CrossRef] 